\title{
DEVELOPMENT OF A SUBJECT SPECIFIC FINITE ELEMENT MODEL USED TO PREDICT THE EFFECTS OF A SINGLE LEG EXTENSION EXERCISE.
}

\author{
A Thesis \\ Presented to \\ The Faculty of the College of Engineering \\ California Polytechnic State University, San Luis Obispo \\ In Partial Fulfillment \\ of the Requirements for the Degree \\ Master of Science in Mechanical Engineering
}

By

Garrett Thomas Gleeson

October 2010 
(C) 2010

Garrett Thomas Gleeson

ALL RIGHTS RESERVED 


\section{COMMITTEE MEMBERSHIP}

TITLE:

AUTHOR:

DATE SUBMITTED:

COMMITTEE CHAIR:

COMMITTEE MEMBER:

COMMITTEE MEMBER:
Development of a Subject Specific Finite Element Model Used to Predict the Effects of a Single Leg Extension Exercise.

Garrett Thomas Gleeson

October 22, 2010
Scott Hazelwood, Ph.D., BME Associate Professor

Andrew Davol, Ph.D., ME Department Chair 


\section{ABSTRACT}

The study presented attempts to prove the concept that mechanical changes in the structure of a bone can be predicted for a specific exercise by a subject specific model created from CT data, MRI data, EMG data, and a physiologic FE model. Previous work generated a subject specific FE model of a femur via CT and MRI data as well as created a set of subject specific biomechanical muscle forces that are required to perform a single leg extension exercise. The FE model and muscle forces were implemented into a single leg extension FE code (ABAQUS) along with a specialized bone remodeling UMAT. The UMAT updated the mechanical properties of the femur via a damage-repair bone remodeling algorithm. The single leg extension FE code was verified by applying walking loads to the femur and allowing the system to equilibrate. The results were used to apply the appropriate walking loads to the final FE simulation for the single leg extension exercise. The final FE simulation included applying the single leg extension loads over a one year period and plotting the change in porosity at various regions of the femoral neck. Although only two regions were found to generate valid results, the data seemed counterintuitive to Wolff's Law which states that bone adaptation is promoted when the material is stressed. The model was successful in creating a subject specific model that is capable of predicting changes in the mechanical properties of bone. However, in order to generate valid FE model results, further understanding of the bone remodeling process and application via a FE model is required. 


\section{ACKNOWLEDGMENTS}

The following work was performed at California Polytechnic State University, San Luis Obispo under the guidance of Dr. Schuster, Dr. Hazelwood, and Dr. Davol. The following research was also aided by previous work performed by Estevan Negrete and Matt Scott. Thank you all for your time and efforts in getting me to the finish line - it was much appreciated. Also, I want to give a special thanks to Dr. Schuster who patiently endured me taking the past three years to complete his research amidst my work and other ever evolving obligations. Last but not least, I would like to thank my mother who is probably more relieved than I am that I am done. Thanks for everything. I am truly grateful. 


\section{Table of Contents}

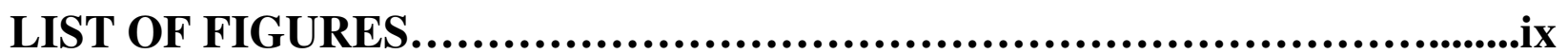

LIST OF TABLES.................................................................

LIST OF COMMON ACRONYMS................................................xiii

CHAPTER 1 - INTRODUCTION.................................................1

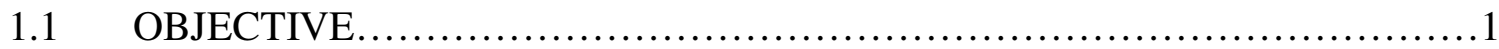

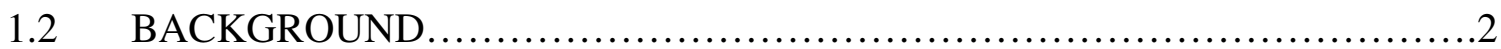

1.2.1 Finite Element Modeling....................................................2

1.2.2 Bone Remodeling: An Overview............................................

1.3 PREVIOIUS WORK: FE MODEL .......................................6

1.4 PREVIOUS WORK: FEMUR FORCE PREDICTIONS .......................8

1.5 MODEL HYPOTHESIS ............................................... 10

CHAPTER 2 - EXISTING RESEARCH...................................11

2.1 BONE REMODELING ALGORITHM.......................................11

2.1.1 Mathematical Models...........................................................11

2.1.1.1 Mechanical Models..........................................13

2.1.1.2 Physiologic Models...........................................17

2.1.1.3 Damage-Repair Models....................................20

2.1.1.4 Summary: Bone Remodeling Algorithm......................24

2.2 PHYSIOLOGIC BOUNDARY CONDITIONS IN FE CODES .................25

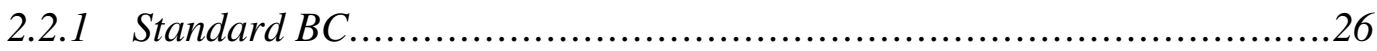

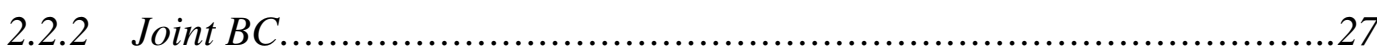

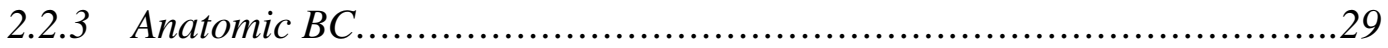

2.2.4 Summary - Physiologic BC............................................... 31

2.3 PHYSIOLOGIC LOADING CONDITIONS IN FE CODES ..................... 31

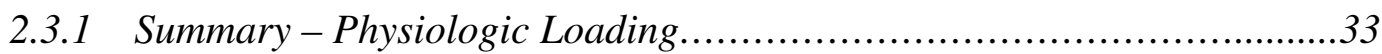

2.4 SINGLE LEG EXTENSION FE MODEL SUMMARY .........................34

CHAPTER 3 - BONE REMODELING ALGORITHM........................35

3.1 REMODELING ALGORITHM: DEVELOPENT .......................... 35

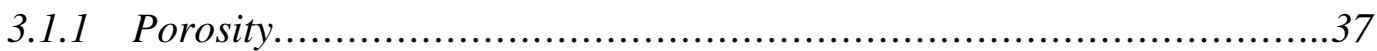

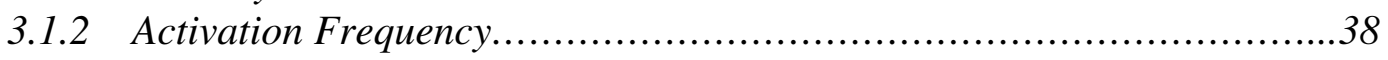

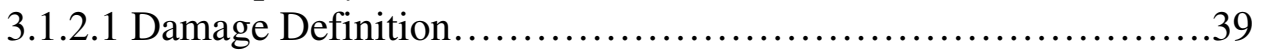

3.1.2.2 Disuse Definition..........................................40

3.1.2.3 Internal Surface Area Definition.............................40

3.1.2.4 Activation Frequency Definition................................41

3.2 REMODELING ALGORITHM - VALIDATION .............................42 


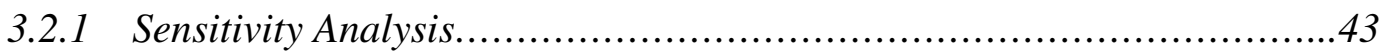

3.2.2 Fatigue Analysis - 2D Model..................................................44

3.3 REMODELING ALGORITHM - LEG EXTENSION MODIFICATIONS......47

\section{CHAPTER 4 - FE MODEL DEVELOPMENT ....................................48}

4.1 THREE POINT BENDING VALIDATION..................................49

4.1.1 Three Point Bending - Experimental Set Up ...................................49

4.1.2 Three Point Bending - Data Acquisition.........................................51

4.1.3 Three Point Bending - FE Model.............................................52

4.1.4 Three Point Bending - Validation Results....................................52

4.2 LOADING AND BOUNDARY CONDITION APPLICATION .................56

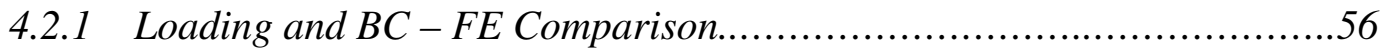

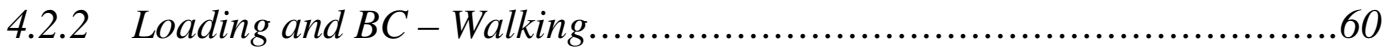

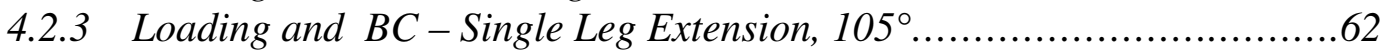

4.2.3.1 $105^{\circ}$ Extension: 3D Femur Muscle Locations.....................63

4.2.3.2 105 Extension: Muscle Force Distribution........................64

4.2.3.3 $105^{\circ}$ Extension: Verify Muscle Distribution Forces in

Equilibrium....................................................65

4.2.4 Loading and BC - Single Leg Extension, 162 ..............................67

4.2.4.1 $162^{\circ}$ Extension: Muscle Force Distribution.......................68

4.2.4.2 $162^{\circ}$ Extension: Verify Muscle Distribution Forces in

Equilibrium...............................................68

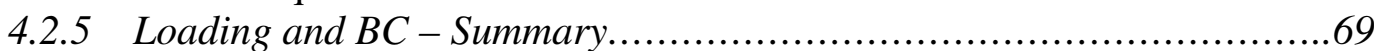

4.3 BONE REMODELING: ALGORITHM.....................................70

4.3.1 Pre-Condition Data Application.................................................... 70

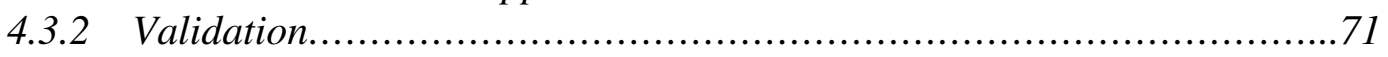

4.3.3 Bone Remodeling Algorithm: Summary ........................................76

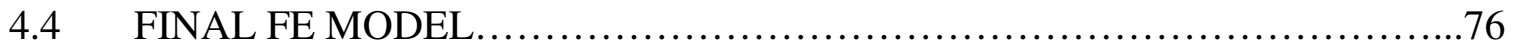

CHAPTER 5 - RESULTS..............................................77

$5.1 \quad$ SINGLE LEG EXTENSION SIMULATION.................................77

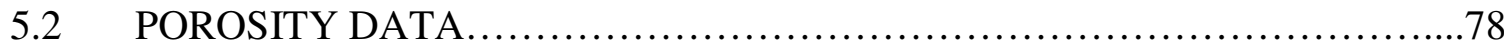

$5.3 \quad$ NET CHANGE IN BONE POROSITY .................................... 82

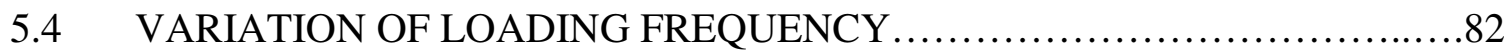

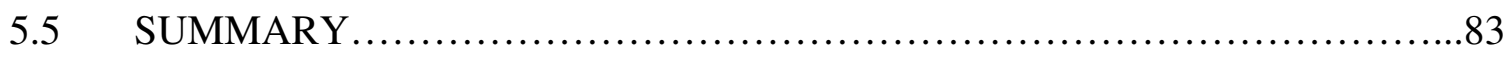

CHAPTER 6 - DISCUSSION.....................................................85

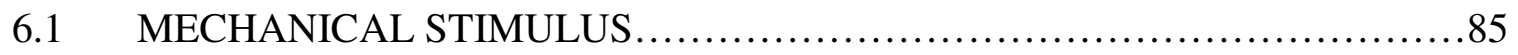

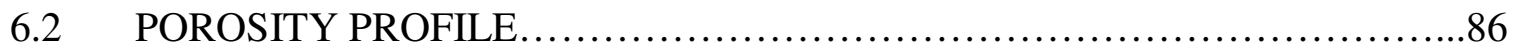

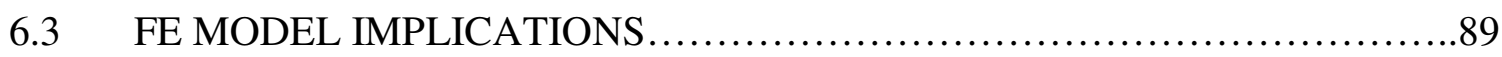


CHAPTER 7 - FUTURE WORK.............................................91

7.1 SINGLE LEG EXTENSION PREDICTED LOADS ..........................91

7.2 SINGLE LEG EXTENSION FE SIMULATION ............................92

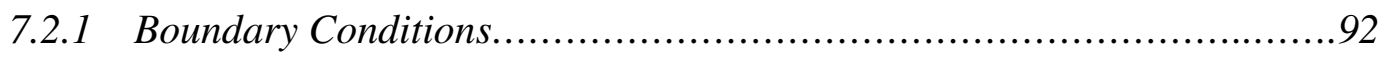

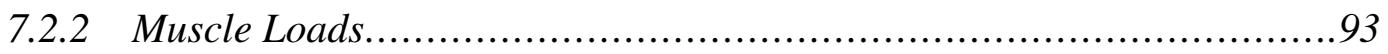

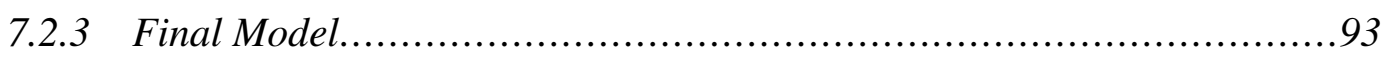

7.3 BONE REMODELING SIMULATION/UMAT ...........................95

CHAPTER 8 - CONCLUSION..........................................97

REFERENCES.............................................................99

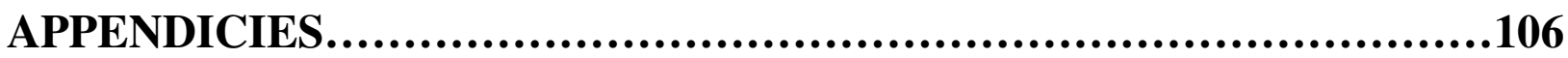

APPENDIX A: MUSCLE FORCE REDISTRIBUTION ............................106

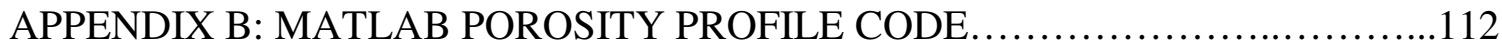

APPENDIX C: ABAQUS INPUT FILE FOR MUSCLE FORCES.................................122

APPENDIX D: HOW TO CREATE A UMAT IN ABAQUS .........................128 


\section{LIST OF FIGURES}

Figure 1-1: Graphical depiction of ARF cycle......................................

Figure 1-2: Resorption location and geometry for BMUs acting in (a) cortical bone and

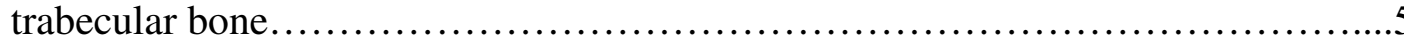

Figure 1-3: Process flow chart for Femur FE model...................................

Figure 1-4: FBD of Femur during Single Leg Extension................................

Figure 1-5: Exercise configurations for the Single Leg Extension.........................10

Figure 2-1: Timeline of major milestones in the creation of the modern bone remodeling

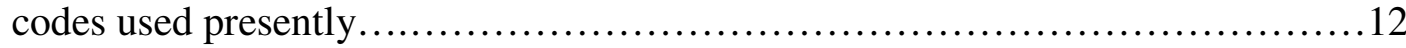

Figure 2-2: Representation of a dead zone in determination of the density rate of change

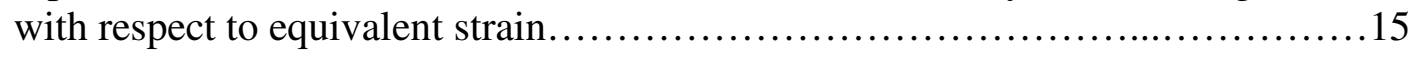

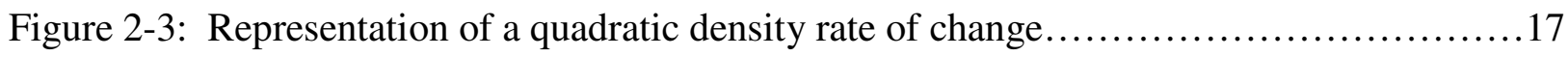

Figure 2-4: Comparison of density distributions for [a] the code developed by Weinanas el al. (1992) and [b] the code developed by Mullender et al. (1994) with the

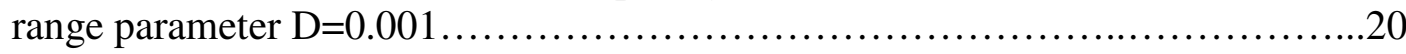

Figure 2-5: Diagram of the continuum damage-repair model of bone remodeling.............23

Figure 2-6: Graphic representation of the three kinds of FE model constraints compared

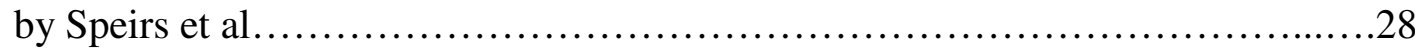

Figure 2-7: Depiction of the muscle and ligamentous anatomic BCs for the femur............30

Figure 3-1: Schematic of Hazelwood's damage-repair bone remodeling algorithm.............37

Figure 3-2: Graphical representations describing (a) $f_{a \text { (disuse) }}$ as a function of $\Phi$ and $f_{a(\text { damage })}$ as a function of $D$

Figure 3-3: Steady state porosity values (a) over an initial 400 day period and (b) at the final 2000 day mark for an initial load of 445.8N

Figure 3-4: Baseline density profile after a 1,200 day simulation using Hazelwood's remodeling algorithm to determine a baseline condition for the marathon regimen comparison.

Figure 3-5: Microdamage levels in various regions of interest within the femur over the 16 week period of the various training regimen

ix 
Figure 4-1: Experimental Three Point Bending set up in an Instron with attached strain gauges.

Figure 4-2: Anterior View of the femur with the approximate locations of strain gauge Rosette B and strain gauge Rosette D. .

Figure 4-3: Force-deflection comparisons between the three point bending experiment and the FE model.

Figure 4-4: Strain-deflection comparisons between the three point bending experiment and the FE model for test run \#4.

Figure 4-5: Three point bending strain rosette B and D values for experimental test runs \#2 through \#6.

Figure 4-6: Anterior view of FE femur model with the maximum strain distributions for each of the three model case, (a) - (c). Strain range: $0.008500 \varepsilon-0.000500 \varepsilon$

Figure 4-7: Applied walking muscles/BC for the single leg extension FE model in the (a) anterior and (b) posterior views: (1) Hip Joint, (2) Gluteus Medius,

(3) Gluteus Minimus, (4) Psoas, (5) Vastus Medialis, (6) Vastus Lateralis,

(7) Adductor Brevis, (8) Adductor Longus

Figure 4-8: Force and BC locations for single leg extension FE model: (1) Vastus Lateralis, (2) Vastus Medialis, (3) Vastus Intermedius, (4) Patella Contact, (5) Medial Condyle Contact, (6) Lateral Condyle Contact, Hip Constraint ( $\left.\mathrm{X}_{\text {hip }}\right)$, and Lateral Condyle Constraint $\left(\mathrm{X}_{\text {condyle }}\right)$....

Figure 4-9: The femoral neck (A) anterior, (B) medial, (C) posterior, and (D) lateral areas of interest for the FE walking model used for validation.

Figure 4-10: Comparison of steady state porosity values in the anterior femoral neck for a FE simulation with walking only loads.

Figure 4-11: Comparison of steady state porosity values in the posterior femoral neck for a FE simulation with walking only loads.... .73

Figure 4-12: Comparison of steady state porosity values in the lateral femoral neck for a FE simulation with walking only loads.

Figure 4-13: Comparison of steady state porosity values in the medial femoral neck for a FE simulation with walking only loads.

Figure 5-1: Anterior Femoral Neck Region: Porosity profile over (A) the 600 day simulation and (B) a year of daily single leg extension exercises at 30cpd, 3,000cpd, and $300,000 \mathrm{cpd}$ 
Figure 5-2: Posterior Femoral Neck Region: Porosity profile over (A) the 600 day simulation and (B) a year of daily single leg extension exercises at 30cpd, 3,000cpd, and

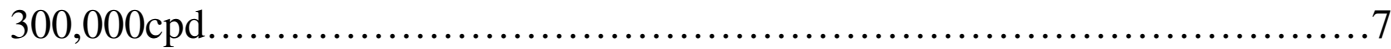

Figure 5-3: Medial Femoral Neck Region: Porosity profile over (A) the 600 day simulation and (B) a year of daily single leg extension exercises at 30cpd, 3,000cpd, and $300,000 \mathrm{cpd}$.

Figure 5-4: Lateral Femoral Neck Region: Porosity profile over (A) the 600 day simulation and (B) a year of daily single leg extension exercises at $30 \mathrm{cpd}, 3,000 \mathrm{cpd}$, and $300,000 \mathrm{cpd}$

Figure 5-5: Comparison of femoral neck porosity after a one year simulation with varying frequencies: $30 \mathrm{cpd}, 3,000 \mathrm{cpd}$, and 300,000cpd.

Figure 6-1: Porosity response (a) over the initial 400 days and (b) at 2000 days for a load of $445.8 \mathrm{~N}$ with varying time increments 


\section{LIST OF TABLES}

Table 3-1: The Hazelwood remodleing algorithm state variables and constants...............36

Table 4-1: Applied $105^{\circ}$ single leg extension loads of the FE model used to compare three

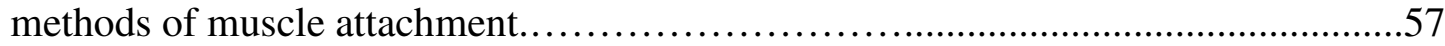

Table 4-2: DOF associated with the Joint BC in ABAQUS FE models........................58

Table 4-3: SPRP, SPNT, \& LPRP hip, lateral condyle, and strain data......................58

Table 4-4: Muscle force magnitudes for 3D loads involving the toe on and heel off phases of

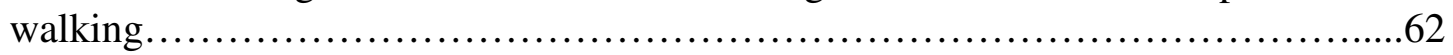

Table 4-5: Muscle force magnitudes for 3D loads involving the toe on and heel off phases of

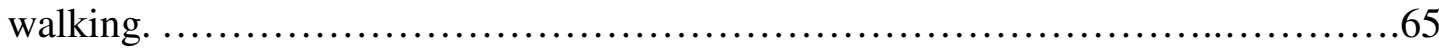

Table 4-6: $105^{\circ}$ extension validation results: $\mathrm{P}=$ Predicted Force, $\mathrm{A}=\mathrm{ABAQUS}$ Force..........67

Table 4-7: Muscle force magnitudes for a 3D $162^{\circ}$ single leg extension.....................68

Table 4-8: $162^{\circ}$ extension validation results: $\mathrm{P}=$ Predicted Force, $\mathrm{A}=\mathrm{ABAQUS}$ Force..........68

Table 4-9: Steady state porosity at $110 \%$ walking loads (simulated time=200 days)..........76

Table 5-1: Net change in bone porosity over a year of daily loading via single leg extension

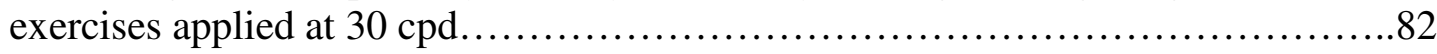




\section{LIST OF ACRONYMS}

$\begin{array}{ll}\text { FE } & \text { Finite Element } \\ \text { BC } & \text { Boundary Condition } \\ \text { CT } & \text { Computed Tomography } \\ \text { EMG } & \text { Electromyography } \\ \text { PCSA } & \text { Physiologic Cross Sectional Area } \\ \text { MRI } & \text { Magnetic Resonance Image } \\ \text { BMU } & \text { Basic Multicellular Unit } \\ \text { ARF } & \text { Activation-Resorption-Formation } \\ \text { SED } & \text { Strain Energy Density } \\ \text { RP } & \text { Reference Point } \\ \text { SPSV } & \text { Small Patch Surface Vector } \\ \text { SPTV } & \text { Small Patch Traction Vector } \\ \text { LPSV } & \text { Large Patch Surface Vector } \\ \text { DOF } & \text { Degree of Freedom } \\ \text { 2D } & \text { Two Dimensional } \\ \text { 3D } & \text { Three Dimensional } \\ \text { cpd } & \text { Cycles per Day }\end{array}$




\section{CHAPTER 1: INTRODUCTION}

\subsection{OBJECTIVE}

As people become older, the natural progression of the human body leads to decreases in muscle and bone mass which can directly affect the quality of life. A decrease in bone mineral density (BMD) can lead to frail bones which are more prone to fracturing, specifically in the wrist and hip, and can lead to further health complications. In addition to age related decreases in BMD, the bone degenerating disease osteoporosis affects over 200 million people worldwide according to the International Osteoporosis Foundation. One proposed method for delaying the loss of BMD has been physical exercise. Exercise has been used as a therapeutic tool in preventing the decrease of BMD in pre- and post-menopausal women. A 1996 study involving $55+$ year old women showed that exercising twice a week led to no net change in BMD as opposed to the control group which had a significant decrease (Hartard et al., 1996). Given the above statements and results, the research presented hereafter attempts to determine if it is possible to mathematically model an increase of BMD via exercise.

Various studies have shown that strength training has various health benefits to help fight off the effects of bone loss due to the aging process and osteoporosis (Menkes et al., 1993, Wolff et al., 1999, Souminen, 2006, Going et al., 2009). Therefore, if the forces from a specific exercise were known and could be applied to a bone in a finite element (FE) model, the output could be used to quantify an increase/decrease in BMD. Previous work has been completed in creating an FE model of a human femur (Negrete, 2008) as well as the forces the leg undergoes during a single leg extension exercise (Scott, 2008). The objective of the following study is to use the aforementioned research to mathematically model a single leg extension exercise with the intent to determine how BMD is affected. If successful, the model could have far reaching 
implementations which include minimizing the decrease of BMD from the aging process, maintaining the BMD of astronauts in a reduced loading environment, prolonging the onset of osteoporosis, and much more.

\subsection{BACKGROUND}

\subsubsection{FINITE ELEMENT MODELING}

Finite element modeling is a very powerful tool which has myriad applications; in the biomedical field alone, it has been used extensively in creating models of the human femur (Cody et al., 1999; Keyak et al., 2003; Shahar et al., 2003; Wirtz et al., 2003; Taddei et al., 2006a, Taddei et al., 2006b, Bessho et al., 2007; Schileo et al., 2007; Yosibash et al., 2007). In the most basic sense, the FE method allows physical phenomena to be modeled via mathematical equations and relations. One reason it is so advantageous is that it can offer relevant results that would have otherwise required physical testing which can be very costly, difficult to measure, and time consuming. Another benefit to the FE method is how easy it can be to vary the system parameters for a particular test in order to observe how different inputs affect the outputs. The ability to produce valid results as well as being able to quickly implement changes in the model parameters make the FE method ideal for simulating a single leg extension exercise in the human femur.

Although the FE method can be very fortuitous for the user, it can easily be used incorrectly which would yield useless results. In order to ensure that an FE model is created accurately and will yield valid results, the user must be knowledgeable in both the FE software program being used as well as the physical phenomena being recreated. Also, a validation of some sort is typically used to ensure that the program is outputting relevant data. Model validations usually 
include a simplified loading scenario (i.e. pure tension, bending, torsion, etc). which is then reproduced physically with the characteristic output used for comparison between the two methods. Once the validation has been completed, the parameters of the model can be manipulated into more complex configurations and loading scenarios in order to solve a more complex problem.

\subsubsection{BONE REMODELING: AN OVERVIEW}

The bone remodeling process is performed by a group of cells acting as a single cohesive unit known as a basic multicellular unit (BMU). BMUs can only become activated on a boney surface but that does not mean they cannot be activated internal to a bone. Bone is a porous material that has an extensive amount of tiny tunnels interwoven throughout which allow for countless surfaces to allow for BMU activity. The two main cell types which make up a BMU are osteoclasts (bone resorbing/removal cells) and osteoblasts (bone formation cells), both of which are derived from precursor cells. Bone remodeling can be broken into three main stages known as activation (A), resorption (R), and formation (F), or ARF for short (see figure 1-2). 


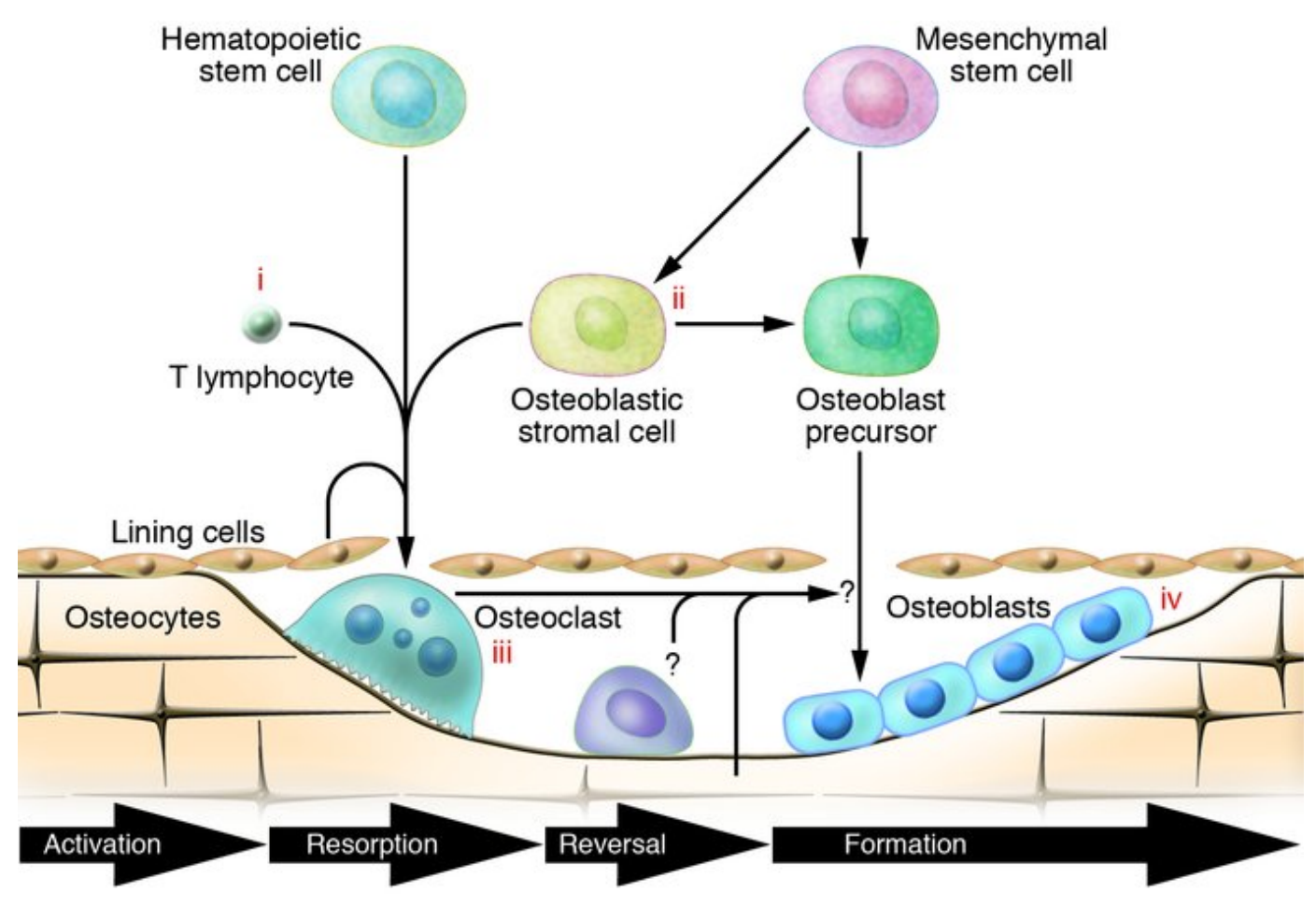

Figure 1-2: Graphical depiction of the ARF cycle. (http://www.jci.org/articles/view/27071/figure/1)

Within the three main stages of ARF there exists six smaller phases which describe the complete cycle.

(1) Activation: Osteoclast cells begin to be created from the differentiation of precursor cells via external stimuli. Although it is not fully understood what the recruitment method is that signals the creation of osteoclasts, a response to stress, strain energy, effective strain, effective stress, and damage have been noted by previous authors (Beaupré et al., 1990a,b, Hill et al., 1998, Garcia et al., 2001, Hazelwood et al., 2001).

(2) Resorption: Once the osteoclasts are fully formed, they begin resorbing the bone ( $40 \mu \mathrm{m} /$ day). Typically in cortical bone the removal process creates a cylindrical cavity internal to the bone, the axis of which is parallel to the long axis of the bone. 
In trabecular bone, the resorbed bone creates a pocket on the external surface of the trabeculae. Figure 1-2 shows each type of resorption for cortical and trabecular bone.
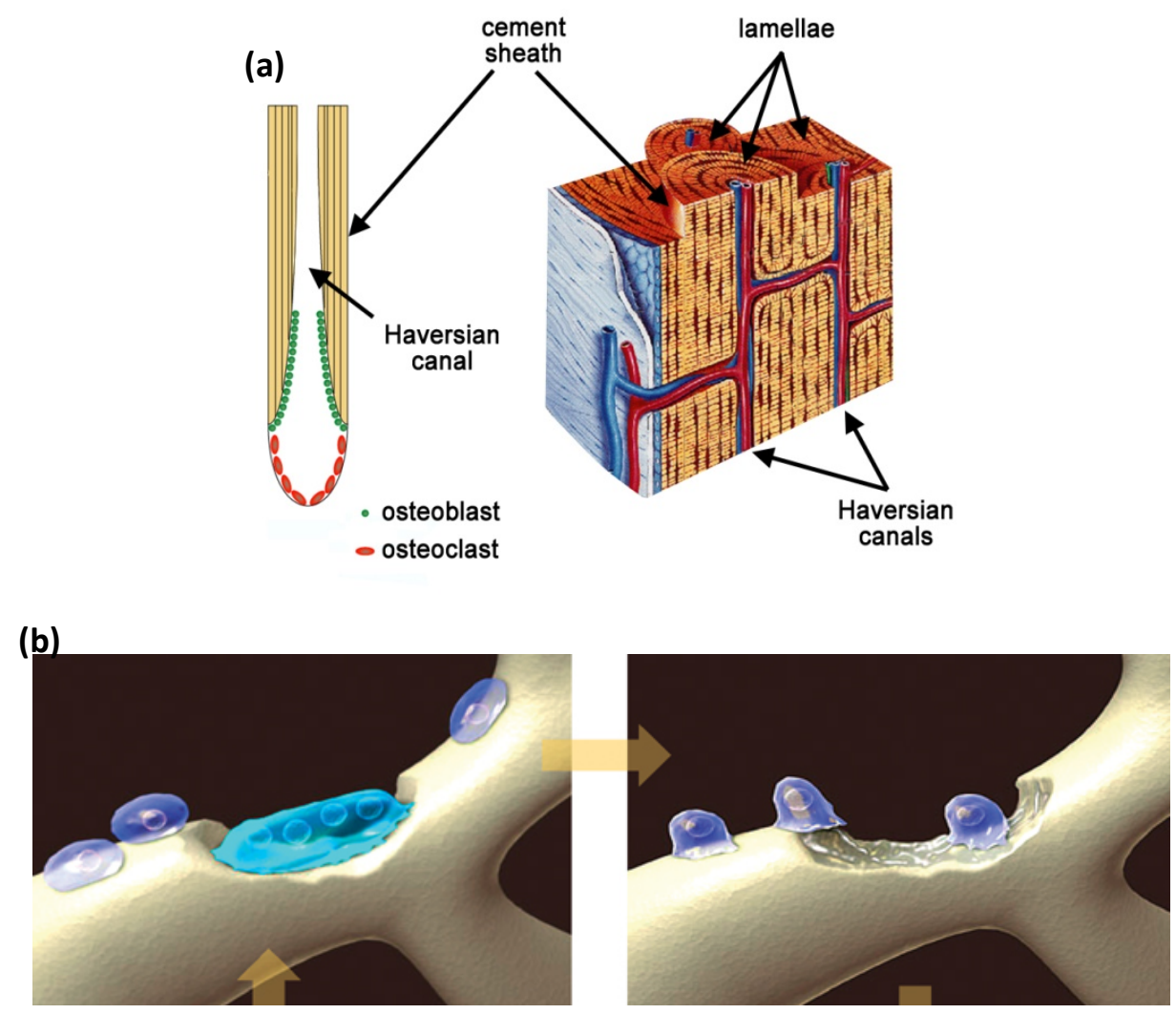

Figure 1-2: Resorption geometry and location for BMUs acting in (a) cortical bone (cylindrical) and (b) trabecular bone (pocket). (http://www.lbl.gov/publicinfo/newscenter/features/assets/img/MSD-bone-tough/Bone-osteon.jpg \&

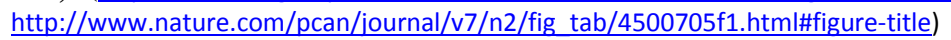

(3) Reversal: This is the transition region between osteoclastic and osteoblastic activity.

The bone has stopped being resorbed by the osteoclasts and cellular signals trigger the recruiting of osteoblast cells to begin forming the new bone.

(4) Formation: The osteoblasts begin to lay down osteoid (unmineralized organic material) which will serve as the scaffolding for the mineralization of the new bone created by the BMU. 
(5) Mineralization: Minerals are deposited in and around the collagen fibers of the osteoid.

(6) Quiescence: Once the resorbing and formation has been completed, the osteoclasts disappear and the osteoblasts either become bone lining cells or disappear as well. *Note: Information for the above steps taken from "Skeletal Tissue Mechanics" by Martin et al.

As expected, each of the phases described above has a certain amount of time associated with it. Although each phase is rate, location, and magnitude specific, the overall time frame for a complete ARF sequence is generally about three and a half to four months long.

It is believed that two of the main functions of bone remodeling are the replacing of micro cracks in order to reduce the chances of bone fatigue failure (Hazelwood et al., 2001) and the optimizing of the mechanical structure in order to adapt to loading conditions (Haapasalo, 1996). However, the knowledge of the intricacies of the bone remodeling phenomena and its biological implications are still being investigated in order to provide more accurate information. As research illuminates the functions and relationships of bone remodeling, the mathematical models presented below will also continue to grow, becoming a more useful diagnostic tool for the future of medicine.

\subsection{PREVIOUS WORK: FEMUR FE MODEL}

The research performed in the following thesis is a continuation of two previous studies: the development of an FE mesh of a human femur bone (Negrete, 2008) and the predicted forces applied to the femur during a single leg extension exercise (Scott, 2008). A brief review of each study is presented here in order to present the assumptions and analysis which directly shape the FE model presented in this study (see Chapter 4). 
The first body of work dealt with the creation of an FE mesh of a human femur bone. It was determined that ABAQUS (ABAQUS/CAE, 2007) would be the FE software utilized for the project and as such, the mesh created needed to output an acceptable format. The process flowchart by which the final femur mesh was created can be seen in Figure 1-3.

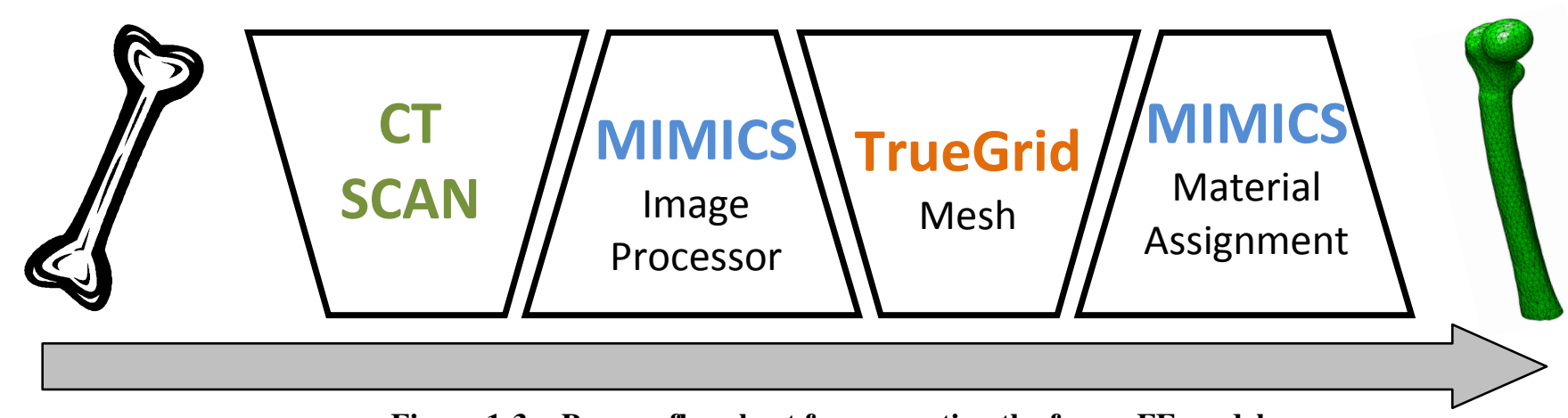

Figure 1-3: Process flowchart for generating the femur FE model.

A computed tomography (CT) scan was performed and then imported into MIMICS (Materialise, 2007) where the slices were reconstructed to create a wireframe shell in the form of the femur. The femur shell was then imported into TrueGrid (TrueGrid, 2006) where an FE mesh was applied to create the individual elements. Once the mesh was completed, the femur was imported into MIMICS once more so the material property of each element could be assigned.

The material property for the final FE femur was created using relationships between Hounsfield Units $(\mathrm{HU})$, apparent density $\left(\rho_{\text {app }}\right)$, and the elastic modulus $(\mathrm{E})$. The CT scan offers a density map of the femur but outputs the data in the form of HUs. The linear relationship between the HUs and the apparent density in equation 1 allows for the material property data for the femur to be converted into the final elastic modulus form through equation 2 (Negrete, 2008). 


$$
\begin{array}{r}
\rho_{a p p}=10^{-2} H U \\
\mathrm{E}=5.966 \times 10^{12} \rho_{a p p}^{1.49}
\end{array}
$$

The transformation of the material property from $\mathrm{HU}$ to $\mathrm{E}$ for the FE femur is necessary because the ABAQUS material property code required to capture the bone remodeling process does so by modifying the elastic modulus. This will be discussed further in Chapter 4.

The final FE femur model also had some key assumptions which are noteworthy in fully understanding the capabilities and limitations of the femur FE model. Based upon a decision matrix (Negrete, 2008) comparing the ease of mesh generation, computational accuracy, geometric resolution of the bone, and computational time, the femur FE model is comprised of hexahedral elements. Also, previous research of an isotropic material model for human bones was found to be nearly identical to an orthotropic model in predicting nodal displacements and stress (Peng et al., 2006). As such, an isotropic material model was incorporated into the femur FE model.

\subsection{PREVIOUS WORK: FEMUR FORCE PREDICTIONS}

The second study focused on determining the various forces applied to the femur during a single leg extension exercise. The forces were predicted using a SimMechanics (Matlab, 2008) computer code. A free body diagram of the femur with the output variables provided by the SimMechanics/MatLab model can be seen in Figure 1.2. 


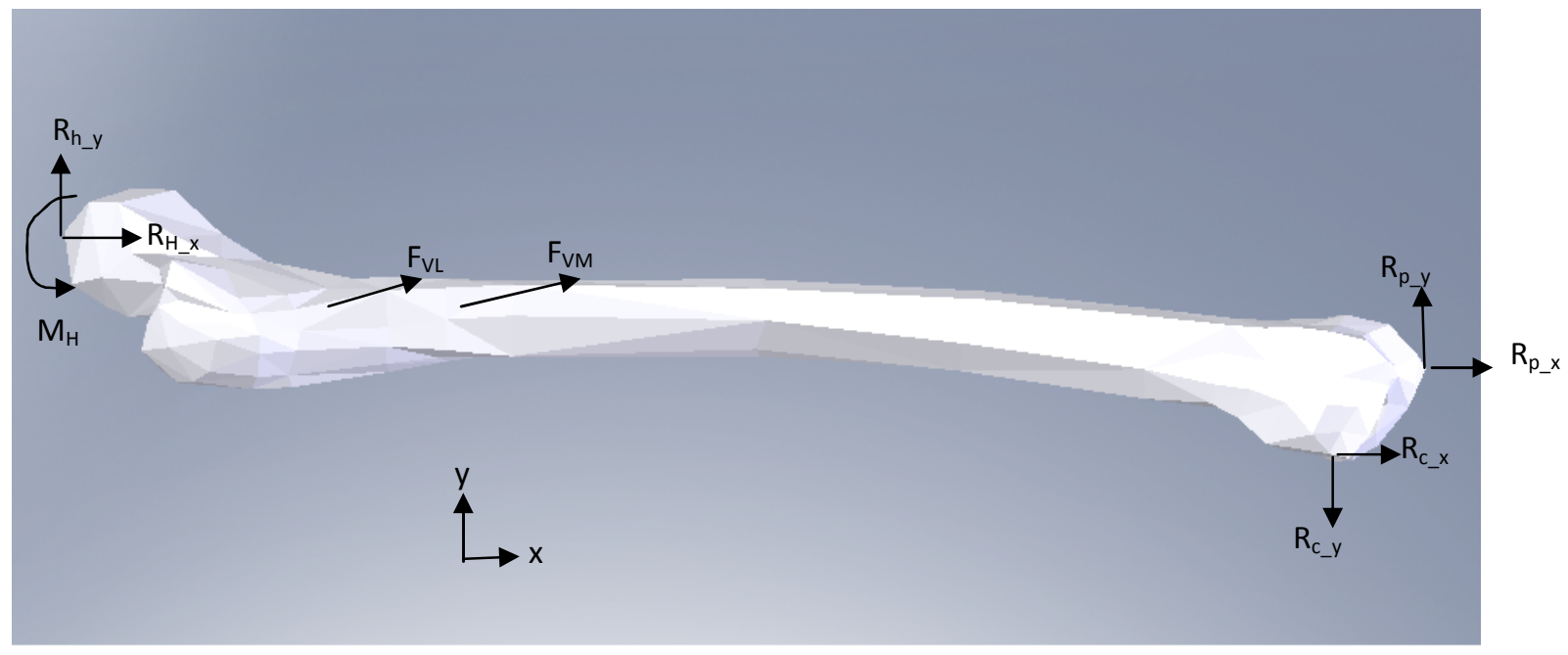

FIGURE 1.4: FBD of SimMechanics femur forces. (Scott, 2008)

The SimMechanics model is able to process electromyography (EMG) data for a single leg extension and output the force magnitudes of the corresponding muscles, tendons, and ligaments in the thigh. EMG data was collected for the three major muscles in the thigh active during a single leg extension - Rectus Femoris, Vastus Lateralis, and Vastus Medialis. It was assumed that these muscles are adequate in representing the muscular activity during a single leg extension (Scott, 2008). The processed EMG data was used to determine the individual muscle activation seen during the exercise. Once the muscle activations were known, the individual muscle forces were determined using a set of force-length curves adapted for each muscle. Normalized force-length curves were scaled to individual muscles using the physiologic cross sectional area (PSCA) of the muscles, which determines the maximum force output.

The forces determined from the force-length curves were then applied to the two geometric configurations seen during the exercise - a $105^{\circ}$ extension and a $162^{\circ}$ extension of the leg. 


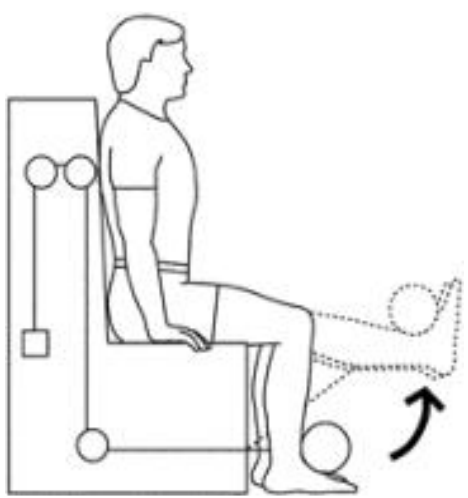

FIGURE 1.5: Single leg extension exercise configurations, $105^{\circ}$ (solid line) and $162^{\circ}$ (dotted line).

Each configuration was run with SimMechanics and output the resulting joint and contact forces during the exercise. The SimMechanics model was validated using external torque data which was recorded during the exercise and was compared directly to the computed outputs with good results (Scott, 2008).

\subsection{MODEL HYPOTHESIS}

The research presented in the following paper is a proof of concept. It is hypothesized that it is possible to predict an individual's BMD response to a single exercise with the use of patient specific information via CT scans, MRIs, EMG data, and a physiologically bounded FE model. If successful, the research would allow doctors, physical therapists, etc. to create highly specialized exercise regimens which would be able to counteract the effects of decreased BMD. 


\section{CHAPTER 2: EXISTING RESEARCH}

An extensive literature search was performed in order to determine the appropriate FE model parameters as well as to see what has been done in the past. There were two main focuses of the literature search. The first area of concern was the aspect of implementing a bone remodeling algorithm into the material property of a FE code. The second area dealt with the determining physiologic boundary conditions as well as loading conditions in the FE code.

\subsection{BONE REMODELING ALGORITHM}

Bone remodeling is a biologic phenomenon that allows the internal and external geometry of a bone to adapt to its loading environment. In special cases, studies have shown that tennis players have an increase in bone mineral content, bone mineral density, and cortical wall thickness in the dominant arm when compared to the non-dominant arm [Haapasalo et al., 1996]. A brief overview of the bone remodeling process will be described before the current state of bone remodeling algorithms used in mathematical modeling of biologic systems is presented.

\subsubsection{MATHEMATICAL MODELS}

Although numerous studies have been presented with numerical algorithms involving bone remodeling [Beaupré et al., 1990a, Beaupré et al., 1990b, Doblaré et al., 2001, Hazelwood et al., 2001, Zernicke et al., 2006, Chen et al., 2007, Li et al., 2007, Baiotto et al., 2009], the subject is still relatively new with the majority of papers being published in the past decade. The bulk of the models created have utilized FE methods and software for verification purposes due to the difficulty in comparing results with in-vivo data. A compressed timeline of the evolution of the 
bone remodeling algorithms that have been implemented over the past couple of decades can be seen in figure 2-1.

\section{Timeline for Bone Remodleing Algorithms since 1990}

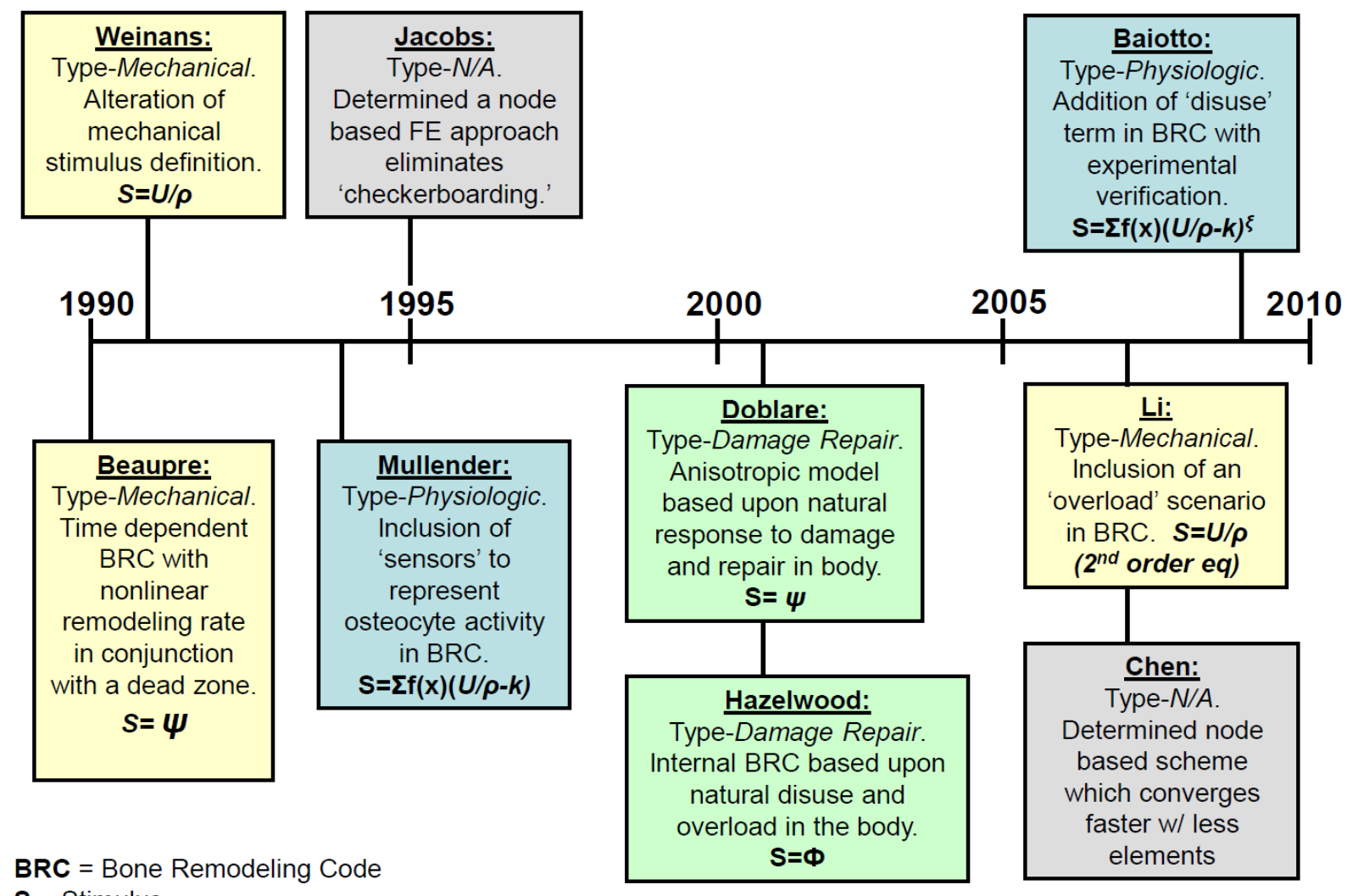

$\mathbf{S}=$ Stimulus

Figure 2-1: Timeline of major milestones in the creation of the modern bone remodeling codes used presently.

The initial research which laid the foundation for the current bone remodeling algorithms observed the strain-remodeling relationship experimentally and reverse engineered simple equations to describe the relationship (Hart et al., 1984). The work by Hart et al. utilized history functions and nth order differential equations to describe the relationship between strain and net bone remodeling. It also implemented the use of a crude computational method which allowed for varying the remodeling parameters in order to see how the model output was affected. 
Although preliminary mathematical remodeling models had been developed since the initial experimental evidence was observed, only the models created in the past 20 years will be discussed in detail. All of the models presented use some form of strain or strain energy density as the stimulus for the remodeling phenomena. Although the mathematical implementation of the strain stimulus is varied, the models can typically be broken one of three distinct types discussed below:

(1) Mechanical Model: It relies heavily on pre-existing bone remodeling data to generate the governing equations of the remodeling phenomena. There is no attempt at incorporating the cellular activity of the ARF cycle and is more of a macroscopic approach to the bone remodeling phenomena.

(2) Physiologic Model: It attempts to capture the microscopic effects of remodeling by incorporating the influence that osteocytes have in remodeling. The model also includes transient responses of remodeling inherent to the ARF cycle.

(3) Damage-Repair Model: It attempts to define the bone remodeling phenomena via the damage-repair relationship of bones. The model incorporates how the effects of damage influence the remodeling response as well as how remodeling can be used to actively remove microdamage in the bone due to loading.

\subsubsection{MECHANICAL MODEL}

The mechanical model acknowledges the presence of some stimulus in which bone is caused to add or remove material. It is heavily based upon previous experimental observations and is implemented mathematically into the remodeling code via a purely mechanical stimulus, 
which does not account for any physiologic phenomena in the bone. One of the first mechanical models proposed (Beaupré et al., 1990a, Beaupré et al., 1990b) had the mathematical form of the following differential equation with respect to time:

$$
\begin{array}{lr}
\dot{\rho}=A\left[\psi\left(\frac{\rho_{c}}{\rho}\right)-\psi_{b A S}\right] S_{v}, & 0 \leq \rho \leq \rho_{c} \\
\dot{\rho}=0, & \text { otherwise }
\end{array}
$$

where

$$
\begin{aligned}
& \psi=\left[\sum_{\text {day }} n_{i} \bar{\sigma}_{i}^{m}\right]^{1 / m} \\
& \bar{\sigma}_{l}=\sqrt{2 E U_{i}}
\end{aligned}
$$

In the above equations $\mathrm{A}$ is a rate constant or function based upon the equivalent strain level, $\rho$ is the apparent density, $\rho_{c}$ is the maximum density of cortical bone, $\psi$ is the continuum level effective stress stimulus, $\psi_{b A S}$ is the attractor state stimulus which represents the value at which there will be no net change in bone density, $S_{v}$ is the surface area per unit volume (and is a function of $\rho), n_{i}$ is the number of daily cycles per load type $i, \sigma_{i}$ is the continuum level effective stress, $m$ is an empirical constant, $E$ is the average Young's modulus, and $U_{i}$ is the continuum level strain energy density (SED). The input parameter of the model is the current apparent density per element, which is then used to determine the SED at each element. The apparent density is also used to calculate the current state of $E$ as well as to populate the matrix of elastic constants (used for the stress-strain relationship). The relationship between $\rho$ and the material properties described depends on the type of material model chosen. The model is advanced to the next instance via the density rate of change equation in conjunction with the forward Euler method (or other time advancing algorithm). The method described above is 
consistent with how FE codes are solved and makes the analysis well suited for FE solver programs such as ABAQUS, Nastran, etc.

The next iteration of this style of bone remodeling code has been implemented by multiple researchers [Huiskes et al., 1992, Weinans et al., 1992, Fyhrie \& Shaffler, 1995, Turner et al., 2005] and has a slightly different stimulus based upon the average elastic energy per unit mass, $S_{e}$. It also contains an additional variable, $s$, to directly create a dead zone in the determination of $\dot{\rho}$ from the equivalent strain value. The dead zone shown in figure 2-2 basically transforms the attractor state stimulus (i.e. where $\dot{\rho}=0$ ) from a singular value to a range of values which is considered to be more physiologically relevant.

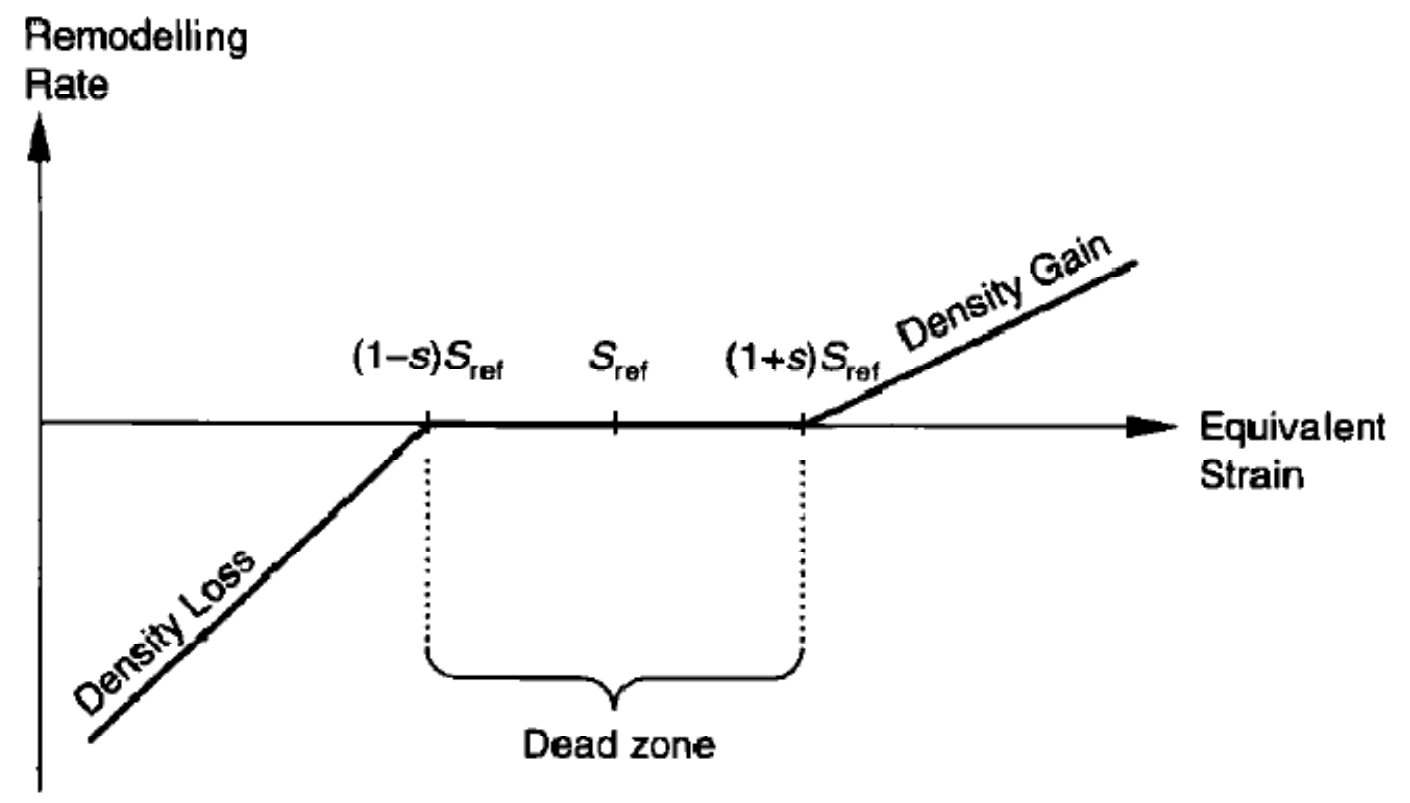

Figure 2-2: Representation of a dead zone in determination of the density rate of change with respect to equivalent strain. (Turner et al., 2005)

The governing equations for this style of remodeling code are piecewise in nature and are as follows: 


$$
\begin{aligned}
& \dot{\rho}=B_{1}\left[S_{e}-(1+s) S_{\text {ref }}\right] S_{v}, \quad S_{e}<(1+s) S_{\text {ref }} \\
& \dot{\rho}=0, \quad(1-s) S_{\text {ref }} \leq S_{e} \leq(1+s) S_{\text {ref }} \\
& \dot{\rho}=C_{1}\left[S_{e}-(1-s) S_{\text {ref }}\right] S_{v}, \quad S_{e}<(1-s) S_{\text {ref }}
\end{aligned}
$$

where

$$
S_{e}=\frac{1}{n} \sum \frac{U_{i}}{\rho}
$$

The variables described in equations 3-5 are still applicable to the variables in equations 6 and 7. The new constants, $B_{1}$ and $C_{1}$, are the slopes of the 'density gain' and 'density loss' lines in figure 2-2, respectively. The slopes can be equal but it has been shown that it may be more clinically relevant to have $B_{1}$ greater than $C_{1}$ (Turner et al., 2005) which means that the removal rate would be faster than the formation rate.

The most recent evolution of the mechanical model includes bone resorption due to an overload condition ( $\mathrm{Li}$ et al., 2007). The advancement was driven by previous research which used a modified piecewise linear function to describe $\dot{\rho}$ (Crupi et al., 2004). The function had a negative slope at some predetermined critical value due to an overload stress value. The addition of an overload value creates a quadratic function of $\dot{\rho}$ over the given stimulus range, shown in figure $2-3$, and therefore adds quadratic terms to equation 6 . The updated equation can be seen as follows:

$$
\begin{aligned}
& \left.\dot{\rho}=B_{1}\left[S_{e}-(1+s) S_{r e f}\right] S_{v}-B_{2}\left[S_{e}-(1+s) S_{r e f}\right]^{2} S_{v}, \quad S_{e}<(1+s) S_{r e f}\right) \\
& \left.\dot{\rho}=0, \quad(1-s) S_{\text {ref }} \leq S_{e} \leq(1+s) S_{\text {ref }}\right\} \\
& \left.\dot{\rho}=C_{1}\left[S_{e}-(1-s) S_{r e f}\right] S_{v}-C_{2}\left[S_{e}-(1-s) S_{r e f}\right]^{2} S_{v}, \quad S_{e}<(1-s) S_{r e f}\right)
\end{aligned}
$$


The variables described in equations 3-5 are still applicable to equation 8 . The constants $B_{1}, B_{2}, C_{1}$, and $C_{2}$ determine the shape and location of the graph seen in figure 2-3.

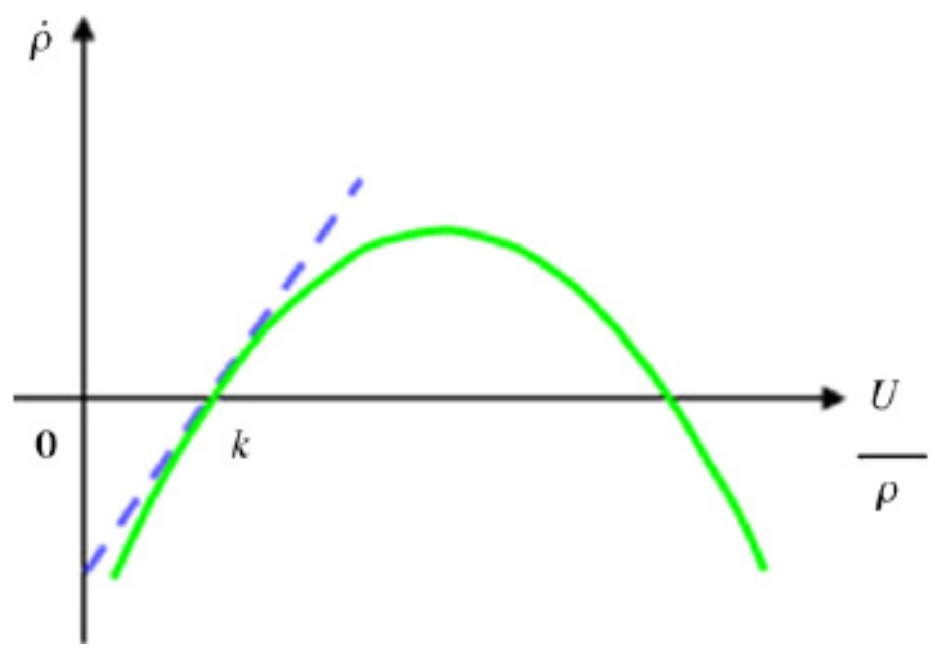

Figure 2-3: Representation of a quadratic density rate of change. The dead zone has been left out for simplicity but could be added if desired. (Li et al., 2006)

\subsubsection{PHYSIOLOGIC MODEL}

The physiologic model attempts to capture the effects that osteocytes have on the ARF cycle. Osteocytes are the most abundant cell found in cortical bone and are interconnected with each other via very small canals called canaliculi. When a microcrack is produced, it can potentially sever the connection between osteocytes and is believed to be a catalyst in initiating the ARF cycle (Lanyon, 1992). The physiologic model attempts to capture the effect that these "sensor" cells have on bone remodeling in order to create a more realistic bone remodeling algorithm.

One of the first models to capture the effects of osteocytic sensor cells (Mullender et al., 1994) was created in order to remove the mesh dependence of the density profile output that is common typical FE codes. The mesh dependence is due to the fact that in typical 
implementations each element is assumed to have only one sensor which is constrained by the elemental boundaries. Therefore, in order to increase the amount of sensors applied to a particular FE model, the mesh must be refined. Another potential issue with the mesh dependent models is the occurrence of checker boarding. Checker boarding happens when the values of a particular elemental mesh can only be defined as empty or fully saturated. This produces discontinuities at the boundary conditions of the elements and is depicted in figure 2-4. The main advantage of the physiologic model is that the number of sensors that are applied to a particular model are independent of the number of elements. Also, the sensors have an effective range, which decreases exponentially with distance, with which they can influence their environment. In effect, the physiologic model is a sophisticated "smoothing" algorithm which aims to remove the discontinuities that can occur within the FE code by averaging multiple sensor values outside the elemental boundaries. The form of the physiologic bone remodeling algorithm can be seen in the following equations:

$$
\dot{\rho}=\tau \phi
$$

where

$$
\begin{gathered}
\phi=\sum f(x)\left(S_{i}-k\right) \\
f(x)=e^{-\left[\frac{d_{i}(x)}{D}\right]} \\
S_{i}=\frac{U_{i}}{\rho_{i}}
\end{gathered}
$$

In the above equations, two new variables are introduced which are not found in any mechanical code; $\tau$ is a time constant or function and $f(x)$ is a decaying exponential function which regulates the influence that the sensors have on the model output. 
Although not as much development of the physiologic model has been performed as the mechanical models, some minor modifications to equations 9-12 have been made which include the effects that unloading, or disuse, have on bones (Baitto et al., 2009). Equations 13 and 14 show the modifications which include adding $\beta$ and $\gamma$ exponent parameters, chosen via experimental data, as well as $\lambda_{\mathrm{j}}$ which accounts for the number of osteocytes in adjacent cells.

$$
\dot{\rho}=\tau \sum \lambda_{j} \phi
$$

where

$$
\phi=\sum f(x)\left[\frac{s_{j}}{\rho_{j}^{\beta}}-k\right]^{\gamma}
$$

Equations 13 and 14 were implemented into an FE code and were placed under a significantly reduced load in order to simulate an environment of microgravity (similar to disuse). The results of the FE model were verified experimentally via rat tibias harvested from tail suspended rats and yielded good agreement with the physical experiment.

The physiologic model is similar to the mechanical model in the sense that a mechanical stimulus, $S_{e}$, is required. However, there are two key components of the physiologic model that allow it to be more biologically relevant than the mechanical model: $\tau$ and its independence of mesh size for a given solution. The time constant allows for the lagging effect created by the ARF process to be captured in the remodeling code. As for the mesh independence, the results by Mullender et al. show how the number of sensors, $\mathrm{N}$, and the effective range parameter, D, can be used to improve an FE model solution without the need for an alternative mesh (Mullender et al., 1994). However, it should be noted that if the number of sensors is chosen to match the number of elements, and the range parameter is sufficiently small, that the results 
would be consistent with a specific FE output where checker boarding is apparent as seen in figure 2-4.

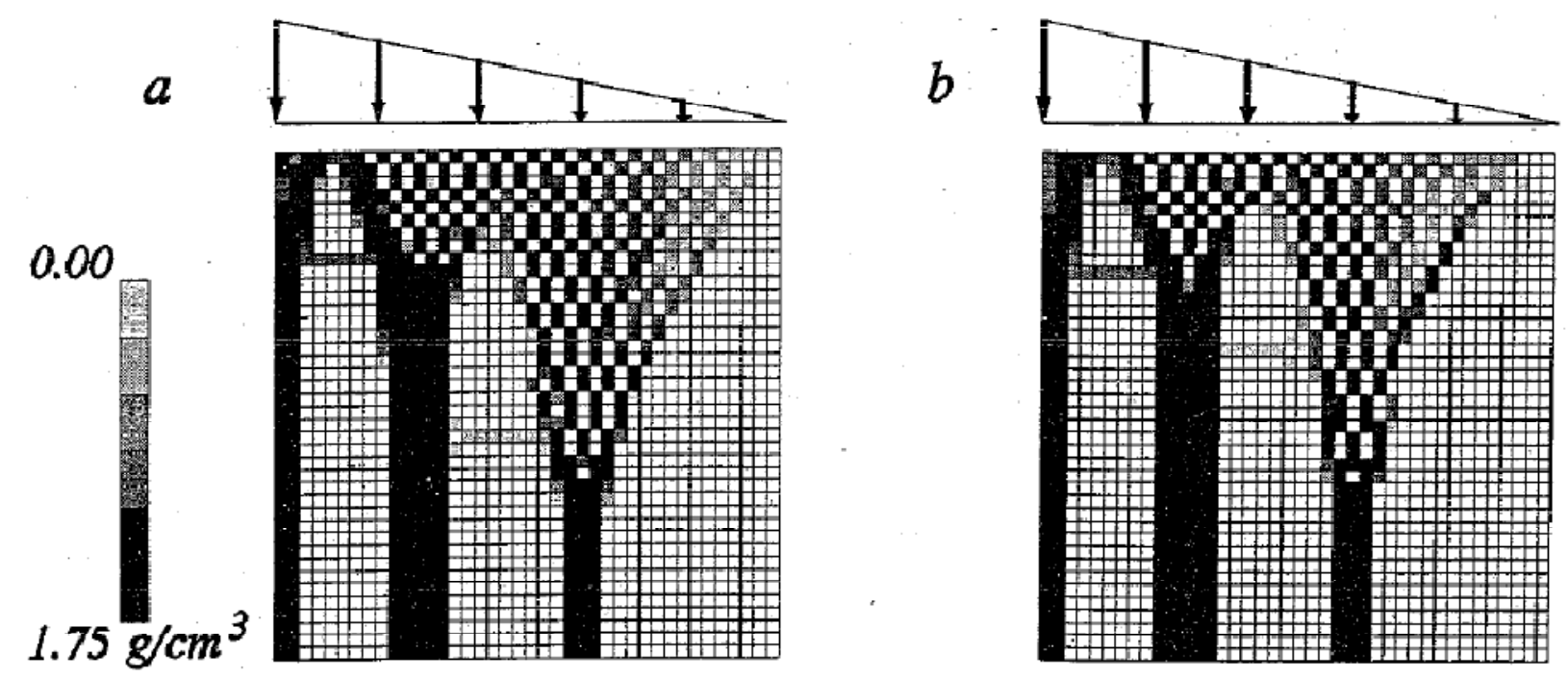

Figure 2-4: Comparison of density distributions for [a] the code developed by Weinanas el al (1992) and [b] the code developed by Mullender et al (1994) with the range parameter $D=0.001$. Both models have the same number of elements and one sensor per element. (Mullender et al., 1994)

The sensor number and range parameter act as a smoothing tool and allow for better model convergence without mesh refinement, something the mechanical model cannot offer.

Therefore, it is safe to say that the physiologic model would be a more powerful tool for clinically relevant studies.

\subsubsection{DAMAGE-REPAIR MODEL}

There are two main damage-repair models which will be discussed (Garcia et al., 2001, Hazelwood et al., 2001) but there is little similarity in how each model is created. The first damage-repair model which was considered was based upon the principals of continuum mechanics (Garcia et al., 2001) and actually generated damage and remodeling tensors for 
incorporating the damage-repair phenomena. The second model generated the parameters for the bone remodeling based upon histomorphometric data (Hazelwood et al., 2001) and is driven by damage formation and removal. Histomorphometric data can be obtained by taking bone biopsies, which have been treated with a contrast dye at known increments of time, and using the information to determine activation frequency, BMU size, and other bone remodeling parameters. Brief explanations of each model are described below.

The continuum mechanics model (Garcia et al., 2001) for the damage-repair bone remodeling algorithm is a very versatile model. Typical remodeling algorithms only account for internal bone remodeling and assume that the bone is an isotropic material. The continuum model presented actually incorporated external remodeling of the bone (referred to as modeling) as well as a scaling factor, $w$, which allows the model to be used assuming a pure isotropic material $(w=0)$ or an anisotropic material $(w=1)$. The algorithm is very complex and contains numerous equations and intermediate steps in order to fully quantify the bone remodeling phenomenon so only a portion of the work will be described below.

First, a damage parameter, $d$, is defined as $0<d<1$ which directly correlates to density of cracks and/or cavities in the bone. In the undamaged state $d=0$, whereas $d=1$ represents a local material failure of the bone. The effective stress $(\bar{\sigma})$ was then defined based on the damage parameter to obtain the isotropic formulation as seen in equation 15 . The anisotropic $\bar{\sigma}$ is shown in equation 16 and is based upon a damage tensor, $D$. Finally, equation 17, which defines the damage tensor in terms of a remodeling tensor, $H$, led to a constitutive bone remodeling tensor for $\bar{\sigma}$, equation 18 .

$$
\bar{\sigma}=\frac{\sigma}{1-d}, \text { Isotropic }
$$




$$
\begin{aligned}
& \bar{\sigma}=(1-D)^{-1 / 2} \sigma(1-D)^{-1 / 2}, \text { Anisotropic } \\
& D=1-H^{2} \\
& \bar{\sigma}=H^{-1} \sigma H^{-1}
\end{aligned}
$$

Next, the free energy function, $\psi$, was used as the stimulus for the model and was a function of both $H$ and the strain. Partial differentiation of $\psi$ with respect to $H$ created the mechanical stimulus tensor, $Y$, shown in equation 19 in terms of strain $(\varepsilon), H$, as well as the Lame parameters $\hat{G}$ and $\hat{\lambda}$.

$$
Y=2\left[2 \widehat{G} \operatorname{sym}[(H \varepsilon H)(H \varepsilon)]+\hat{\lambda} \operatorname{tr}\left(H^{2} \varepsilon\right) \operatorname{sym}[H \varepsilon]\right]
$$

The last two parts of the continuum model, include a tensor, $J$, which determines the influence of the octaedric and deviatoric portions of the remodeling stimulus via the $w$ scale factor, and the rate equations for $H$. The rate equations for $H$ - one for resorption and one for apposition - were based upon available bone surface area, a remodeling function that includes a dead zone, porosity, and the $J$ tensor. Figure 2-5 shows the diagram of how the anisotropic continuum model is used to update the stress for a given FE application. 


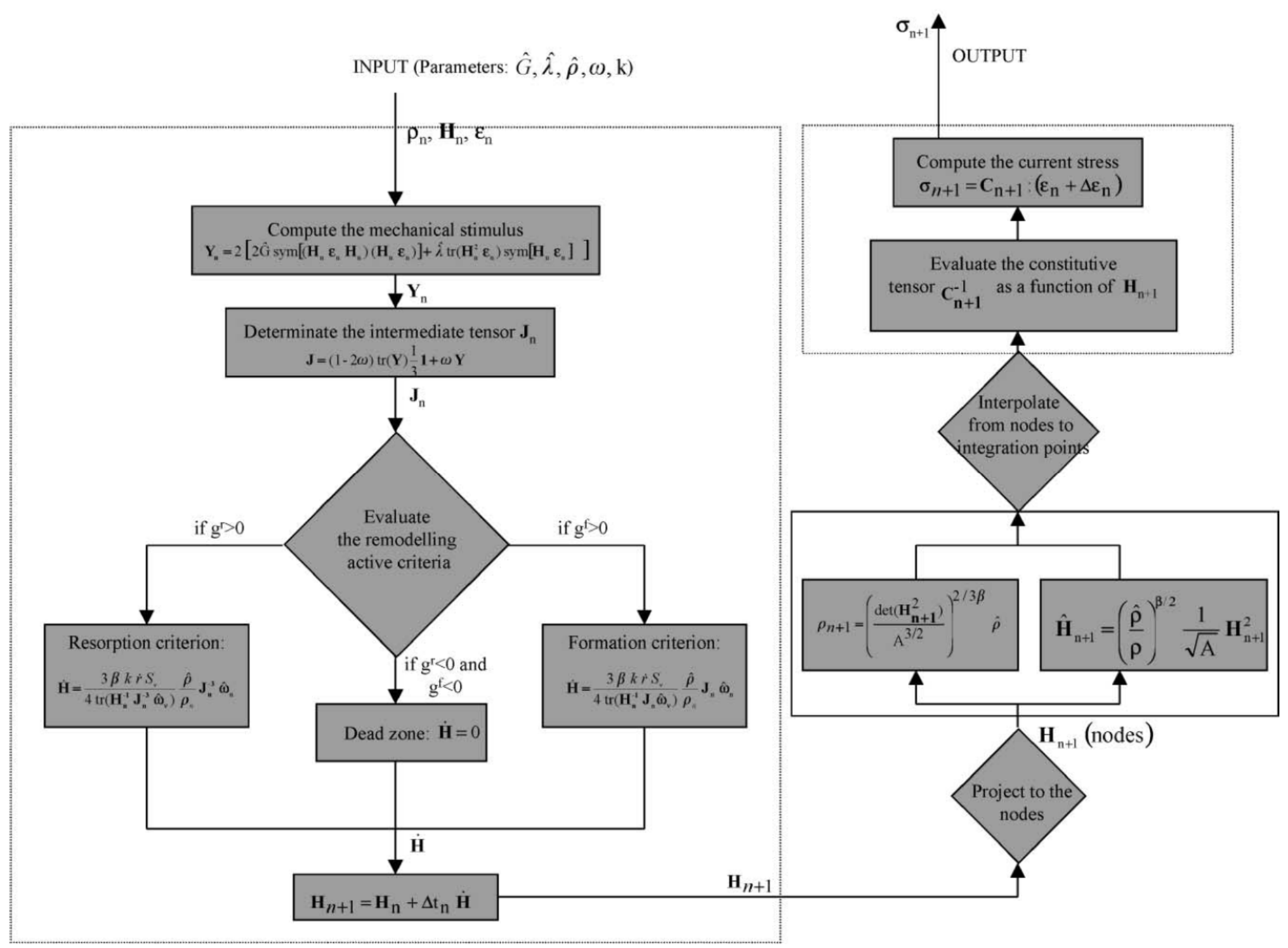

Figure 2-5: Diagram of the continuum damage-repair model of bone remodeling. (Doblare et al., 2001) 
The histomorphometric model (Hazelwood et al., 2001) incorporated the damage-repair phenomenon through various histomorphometric data - activation frequency, BMU crosssectional area, ARF cycle time, etc. The details of the model are discussed in detail in Chapter 3 but are succinctly described as follows. The model is driven by the activation frequency of BMUs in the bone and included the time lag effects of the ARF cycle. Activation frequency is based upon three things: the amount of damage, disuse, and available surface area. The damage is in part determined from the mechanical stimulus, which is based upon the strain distribution for a given load profile. The model outputs the change in porosity profile for the bone which is used to update the Elastic modulus of the bone and capture the effects of remodeling.

\subsubsection{SUMMARY - BONE REMODELING ALGORITHM}

All three bone remodeling algorithms require some amount of experimental data in order to be used in a validated model. The limitation of the mechanical and physiologic models is that they use previously collected data to try and validate the mathematical formulas used to describe the remodeling activity in bones. This means that the remodeling formulas that are eventually determined may only be valid for a particular loading magnitude and scenario (i.e. the gait cycle or stair climbing). The damage-repair models attempt to validate the remodeling process. In the continuum model this is performed by defining a remodeling tensor, and in the histomorphometric model it is done via histomorphometric parameters. By validating the process rather than a specific loading scenario, the damage-repair models above can potentially be used to predict changes in a bone's structure based upon a variety of loading scenarios. In particular, it can predict changes in a single leg extension exercise. Therefore, a damage-repair 
bone remodeling algorithm will be chosen for the development of a single leg extension FE model.

\subsection{PHYSIOLOGIC BOUNDARY CONDITIONS IN FE CODES}

As important as it is to determine an accurate material code in FE models, the proper application of BCs is paramount. This statement bears more weight when applied to bones, for two reasons. First, the material property dependence upon the stress-strain field output, which is required to update the density and elastic modulus at each time step, means that even small variations from the "proper" stress-strain field could create very different results over a given time period. Secondly, bone and its surrounding environment (i.e. tissue, muscles, ligaments) are not so simply modeled. For example, ligaments can be very pliable one second and almost rigid the next depending on the application. Therefore, the assumptions made in determining the proper application of boundary conditions should be well understood in order for the most physiologically relevant FE model to be created.

In an ideal scenario, when analyzing a static loading situation (i.e. holding a dumbbell at a $90^{\circ}$ ) the proper force magnitudes and directions for every muscle and ligament would be known. This would lead to not needing any BCs or constraints because every muscle, tendon, and ligament would be perfectly balanced to keep the bone in its static position. This type of BC is what is known as a 'free' $\mathrm{BC}$ and is very difficult to implement. The required data would be

extremely hard to obtain in vivo and would be equally as hard to implement in any sort of generic model (e.g. a lower limb that could be used for multiple individuals). Muscle and ligament size, shape, and attachment points vary ever so slightly, and in some cases not so slightly, from individual to individual so that a truly generic model could never really exist. Therefore, if the free $\mathrm{BC}$ is the ultimate goal for a static bone model, the following paragraphs consider the other 
BCs previously implemented in physiologic femur FE models as well as their success and/or failure.

\subsubsection{STANDARD BC}

Although the type of BCs chosen for a particular FE model depends on the area of interest for a specific model, there are two standard BC styles which are typically used for constraining the femur.

(1) Distal BC: This type of femoral BC is implemented with the nodes in the femoral condyles being fully constrained and is typically used when the muscle and joint loads are applied at the proximal femur. Standard applications include gait analysis where the loading at the hip joint is of utmost importance. This BC is also used in FE models of specific experiments where the condyles have been set in some sort of cement or clamp and the femoral head and neck are being loaded manually.

(2) Diaphyseal BC: Similar to the distal constraint, this type of femoral BC is used for FE models with loading applied primarily at the proximal femur but has the nodes in the mid-shaft of the femur fully constrained. The diaphyseal $\mathrm{BC}$ is also used to duplicate specific experiments where the distal portion of the bone has been removed the diaphysis is cemented or clamped in place.

The preference for using either the distal or diaphyseal BCs varies depending on what the FE model is simulating. However, the one major disadvantage of each style of $\mathrm{BC}$ is the potential for unrealistic deflections at the femoral head due to the lack of a constraint at that 
location. This is not as prevalent in 2-D models where the forces are in-plane but becomes more of an issue in implementing the forces in a 3-D model.

\subsubsection{JOINT BC}

A recent joint constrained BC for the femur has been developed (Speirs et al., 2006) and has had success in producing physiologically relevant results for FE models of the femur (Speirs et al., 2006, Behrens et al., 2009). The authors compared commonly implemented BCs from previous FE models against a proposed joint BC. They also varied the number of muscles active in the FE model to see how a reduced load condition affects the model strain output. For the BC comparison, all three $\mathrm{BC}$ types were implemented with a complete muscle set. The three types of BCs he considered can be seen in figure 2-6 and are described as follows:

(1) Diaphysis: The FE model is fully constrained at three separate nodes located in the mid-diaphysis of the femur.

(2) Condyles: The FE model is fully constrained at three separate nodes located in the epicondyles of the femur.

(3) Joint: The FE model is bounded by a node at the center of the knee being constrained in all three translational degrees of freedom (DOF), a node at the hip joint contact (i.e. where the joint contact force is applied) being constrained in two translational DOF (allowing it to only translate along the axis toward the knee center, and a node on the distal portion of the lateral condyle was constrained a single DOF constraining Anterior-Posterior motion (allowing no rotation of the FE model about the hip-knee axis). 

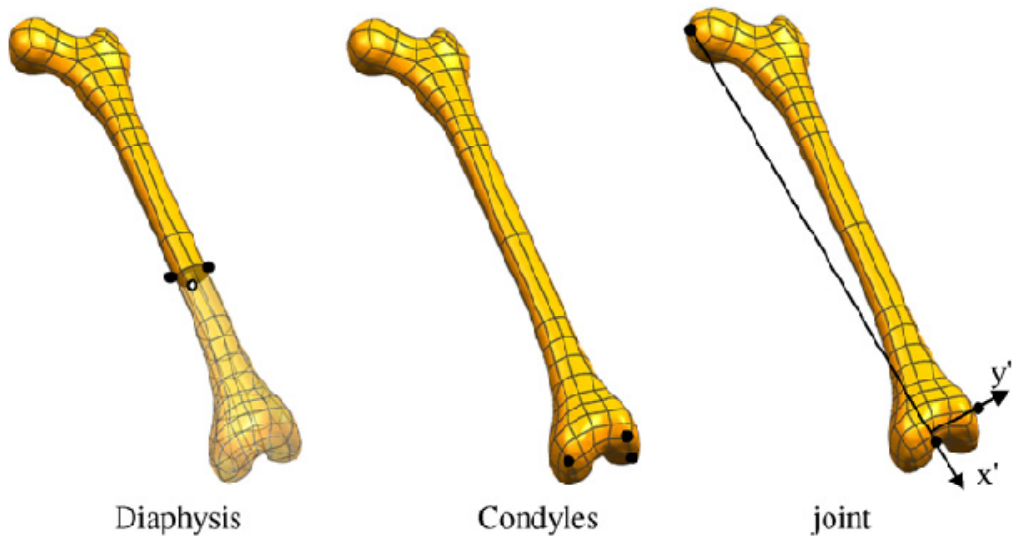

Figure 2-6: Graphic representation of the three kinds of FE model constraints compared by Speirs $e t$ al. (Spiers et al., 2006)

The result of the research concluded by Speirs et al. was that the diaphyseal and condylar constraints alone lead to many erroneous results of the FE model. The reaction loads in both cases were well above the normal range for gait, the deflections at the femoral head were not physiologically comparable to published experimental data, and consequently the surface strains of the bone were much higher than the joint constrained model. This is an important result to note going forward for the final single leg extension FE model because of the strain dependency of the bone remodeling code. If the recorded strain values are artificially increased, it would directly affect the results predicted by the final femur FE model. Not only did the joint constrained model outperform the other two, the femoral deflection and joint reaction forces also agreed well with published experimental data (Taylor et al., 1996). Based upon the close physiologic agreement of the deflection, it was assumed that the joint constrained model also produced a physiologic strain field for the femur. 


\subsubsection{ANATOMIC BC}

An advanced set of anatomic BCs was developed by A.T.M Phillips in his search for an FE model which behaved identical to the in vivo situation - constrained by bones, muscles, ligaments, and tendons only (Phillips et al., 2007, Phillips, 2009). The FE model created by Phillips includes rigid artificial structures representing the spinal vertebral region of L5-S1 (lower limb global origin), the pelvis' acetabular region (proximal femoral constraint) and the tibial plateau region (distal femoral constraint). These structures not only provide a more physiologic anatomic constraint system for the femur, but they also provide the attachment sites for the muscles and ligaments. Also, smooth surface interactions were assigned to the hip and knee joint surfaces to mimic the effects of cartilage. Once the alignment of the pelvis, femur, and tibia were determined, the application of 26 femoral muscles and seven ligaments were applied to the model via spring (connector) elements. Figure 2-7 depicts what the model looks like after the application of the spring elements. 


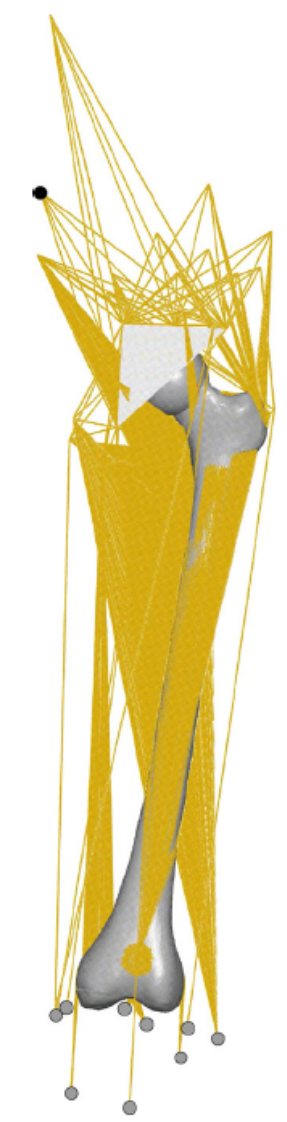

Figure 2-7: Depiction of the muscle and ligamentous anatomic BCs for the femur. (Spiers et al., 2009)

The spring elements are nonlinear representations of how the muscles and ligaments respond to tensile and compressive loads. The governing equations used for this type of FE model are too in depth to go into here but are fully described by Phillips his previous work (Phillips, 2005). The model was verified by applying a load representative of a single leg stance to the L5-S1 vertebral unit and comparing the femoral displacements and strains to experimental data. The FE model agreed well with the data and is currently being developed in order to create a full lower limb model. 


\subsubsection{SUMMARY - PHYSIOLOGIC BOUNDARY CONDITIONS}

The physiologic agreement that the joint and anatomic $\mathrm{BC}$ models posses allow them to become useful tools in developing the FE model for a single leg extension. The constraints of the Speirs model are easier to implement than the Phillips model - constraining three nodes as opposed to completely defining all of the muscles and ligaments of the femur. Also, the Speirs model has been successfully implemented by other authors (Behrens et al., 2009) whereas the Phillips model has only been used in a singular study to date. Although aspects of each model will be used to determine how to physiologically constrain the femur during a single leg extension, the lack of data required for the anatomic $\mathrm{BC}$ means that the joint $\mathrm{BC}$ is best suited for the current study.

\subsection{PHYSIOLOGIC LOADING CONDITIONS IN FE CODES}

Once the BCs have been established, the next step in developing a FE model is to define the loads which will act on the model. As with the boundary conditions, the forces applied to a FE model are crucial to generating a valid model. Depending on how the muscle forces are implemented, an invalid stress-strain field can be generated over the length of the simulation which would create erroneous results. In this section, the various methods of applying femoral muscle forces in FE models is discussed along with the effects they can have on the models' accuracy.

The geographic location of the applied forces for muscles in the leg is important for creating a realistic FE model. Before the geographic coordinates of muscles in the femur was compiled in a single comprehensive body of work (Brand et al., 1982, Brand et al., 1994), experimental data on the actual attachment sites for muscles in the lower extremity either did not exist or were 
not available for general use. Prior to the work of Brand et al, the majority of researchers had to use anatomic texts, such as Grey's Anatomy $y_{2}$ or their own cadavers in order to estimate the proper muscle placement. Brand's work was paramount because is served as the cornerstone for future FE models of the leg (Hoy et al., 1990, Cristofolini et al., 1995, Duda et al., 1997, Duda et al., 1998, Simões et al., 2000, Heller et al., 2001, Stolk et al., 2001, Bitsakos et al., 2005) by creating a scaling method which allowed one set of data to be adapted to multiple femurs. His research also led to studies of the sensitivity of the muscle attachment sites (Brand et al., 1982, Duda et al., 1996). The results of the sensitivity analyses concluded that even small changes in a muscle's location can create unrealistic moments at the joints. However, the problem is mitigated if the applied loads are balanced. Therefore, even though muscle location is important for a good FE model of the femur, obtaining the relevant amount of balanced muscle forces is what will ultimately determine a model's success.

In order to create a physiologically loaded FE model, it is imperative that the active muscles be properly identified along with their corresponding magnitudes. Not only will the active muscles aid in creating a balanced femur FE loading condition, but they will also simultaneously create a physiologically relevant stress-strain distribution. Multiple studies have shown that FE models with simplified muscular loading lead to unrealistic strain fields and stress modes (i.e. bone primarily in bending as opposed to compression) (Cristofolini et al., 1995, Duda et al., 1997, Duda et al., 1998, Simões et al., 2000, Polgar et al., 2003). However, simplified load cases have been implemented with success but are either site specific (i.e. proximal femur only) (Stolk et al., 2001) or involve an additional BC constraint which creates a more physiologic response (Simões et al., 2000). That being said, FE models yielding the best results have a complete muscle force profile which is balanced. 
Lastly, the muscle-bone interaction within the FE models needs to be understood in order to create a truly representative load case. The majority of FE models apply the muscle force as a point load directly to the bone in the approximate geometric location (Hoy et al., 1990, Duda et al., 1996, Duda et al., 1997, Duda et al., 1998, Simões et al., 2000, Heller et al., 2001, Stolk et al., 2001), with sufficient results. However, one particular study uniformly distributed the muscle force over the attachment area of the bone and found the results agreed well with in vivo data (Polgar et al., 2003). Intuitively, it seems appropriate to distribute the load over the attachment site especially in an FE code where point loads often produce unrealistic stress concentrations. However, different muscles respond in different ways depending on the loading; during gait, the gluteus medius muscle fiber activation fluctuates (i.e. anterior fibers may be active when the posterior ones are not and vice versa) (Soderberg et al., 1978).

\subsubsection{SUMMARY - PHYSIOLOGIC LOADING}

Without in-depth knowledge of each muscle's specific geometric location and contractual characteristics, it would be extremely difficult to accurately implement muscle loads in the femur. Muscle locations for the single leg extension FE model will be based upon a combination of previously collected data (Brand et al., 1982) as well similar FE models that have output valid strain results in the femur (Cristofolini et al., 1995, Deuel, 2007) As for how the muscle forces are applied to the FE femur, a distributed force over the muscle attachment site will be implemented in order to reduce the effects of stress concentrations within the model which would adversely affect the bone remodeling algorithm output. 


\subsection{SINGLE LEG EXTENSION FE MODEL SUMMARY}

Based upon the available femur muscle data for the single leg extension FE model, the following parameters will be used to develop the model: a damage-repair bone remodeling algorithm, a set of joint constrained BCs, and muscle forces distributed over the muscle attachment sites of the femur. The implementation of each of these parameters will be discussed in detail in the FE model development of chapter 4. 


\section{CHAPTER 3: BONE REMODELING ALGORITHM}

The final single leg extension FE model will utilize the damage-repair bone remodeling algorithm developed by Hazelwood et al. (Hazelwood et al., 2001). Hazelwood's code allows for the time dependent phenomena of the ARF cycle to be fully captured as well as future validation to be performed because the model parameters are based upon experimentally measureable data. He has graciously allowed his code to be modified for the development of the single leg extension FE model which removes the need for developing a separate model from scratch. Also, the code has previously been validated in an application of femoral fatigue damage analysis for marathon training regimens (Hazelwood et al., 2007). The advantage of implementing an already validated code is that the results obtained in the single leg extension FE model will bear more weight in proving/disproving the hypothesis, that an FE model of a particular exercise can predict the BMD change of the bone undergoing the exercise.

The remainder of this chapter will discuss in detail the development of Hazelwood's bone remodeling code, how it was verified, and how it will be modified in order to reproduce the load scenario for a single leg extension.

\subsection{REMODELING ALGORITHM - DEVELOPMENT}

The driving factors behind the creation of a damage-repair model (Hazelwood et al., 2001) are twofold: to create a model that can be experimentally validated and to expose effects and relationships within remodeling which simpler models do not have the capability of determining (i.e. effect of remodeling space porosity on mechanical function). Unlike the previously discussed mechanical and physiologic models, Hazelwood's code is based upon parameters which can be experimentally measured via histomorphometric data from a specific bone (see 
table 3-1 for a complete list of model variables/constants). Histomorphometric data can be obtained by taking bone biopsies, which have been treated with a contrast dye at known increments of time, and using the information to determine activation frequency, BMU size, and other bone remodeling parameters. Therefore, Hazelwood's code can be validated with physical testing and has the potential to create a much more accurate model. This is almost impossible in the mechanical and physiologic cases, which modify the mathematical constants of the governing equations to correlate to previously collected data averages. A schematic of the bone remodeling code created can be seen in figure 3-1 along with the various parameters which will be discussed more in depth in the following pages.

Table 3-1: The Hazelwood remodleing algorithm state variables and constants. (Hazelwood et al., 2001)

\begin{tabular}{|c|c|c|}
\hline & State variable & \\
\hline E & Elastic modulus (MPa) & \\
\hline$p$ & Porosity & \\
\hline$N_{\mathrm{R}}$ & Number of resorbing BMUs (BMUs/ $/ \mathrm{mm}^{2}$ ) & \\
\hline$N_{\mathrm{F}}$ & Number of refilling BMUs (BMUs $/ \mathrm{mm}^{2}$ ) & \\
\hline$f_{\mathrm{a}}$ & BMU activation frequency (BMUs $/ \mathrm{mm}^{2} /$ day) & \\
\hline$S_{\mathrm{A}}$ & Normalized specific surface area & \\
\hline$D$ & Damage $\left(\mathrm{mm} / \mathrm{mm}^{2}\right)$ & \\
\hline$s$ & Strain, $\mu \varepsilon$ & \\
\hline \multirow[t]{2}{*}{$\Phi$} & Mechanical stimulus (cpd) & \\
\hline & Constant & Nominal value \\
\hline$A$ & Cross-sectional area of each BMU $\left(\mathrm{mm}^{2}\right)$ & $2.84 \times 10^{-2}$ \\
\hline$T_{\mathrm{R}}$ & Resorption period (days) & 24 \\
\hline$T_{\mathrm{I}}$ & Reversal period (days) & 8 \\
\hline$T_{\mathrm{F}}$ & Refilling period (days) & 64 \\
\hline$k_{\mathrm{D}}$ & Damage rate coefficient $\left(\mathrm{mm} / \mathrm{mm}^{2}\right)$ & $1.85 \times 10^{5}$ \\
\hline$n$ & Loading conditions & 1 \\
\hline$q$ & Damage rate exponent & 4 \\
\hline$R_{\mathrm{L}}$ & Loading rate (cpd) & 3000 \\
\hline$F_{\mathrm{s}}$ & Damage removal specificity factor & 5 \\
\hline$D_{0}$ & Initial damage $\left(\mathrm{mm} / \mathrm{mm}^{2}\right)$ & 0.0366 \\
\hline$f_{\mathrm{a} 0}$ & Initial BMU activation frequency (BMUs $/ \mathrm{mm}^{2} /$ day) & 0.00670 \\
\hline$\Phi_{0}$ & Initial mechanical stimulus (cpd) & $1.875 \times 10^{-10}$ \\
\hline$f_{\mathrm{a}(\max )}$ & Maximum BMU activation frequency (BMUs/ $\mathrm{mm}^{2} /$ day) & 0.50 \\
\hline$k_{\mathrm{b}}$ & Activation frequency dose-response coefficient $\left(\mathrm{cpd}^{-1}\right)$ & $6.5 \times 10^{10}$ \\
\hline$k_{\mathrm{c}}$ & Activation frequency dose-response coefficient (cpd) & $9.4 \times 10^{-11}$ \\
\hline$k_{\mathrm{r}}$ & Activation frequency dose-response coefficient & -1.6 \\
\hline
\end{tabular}




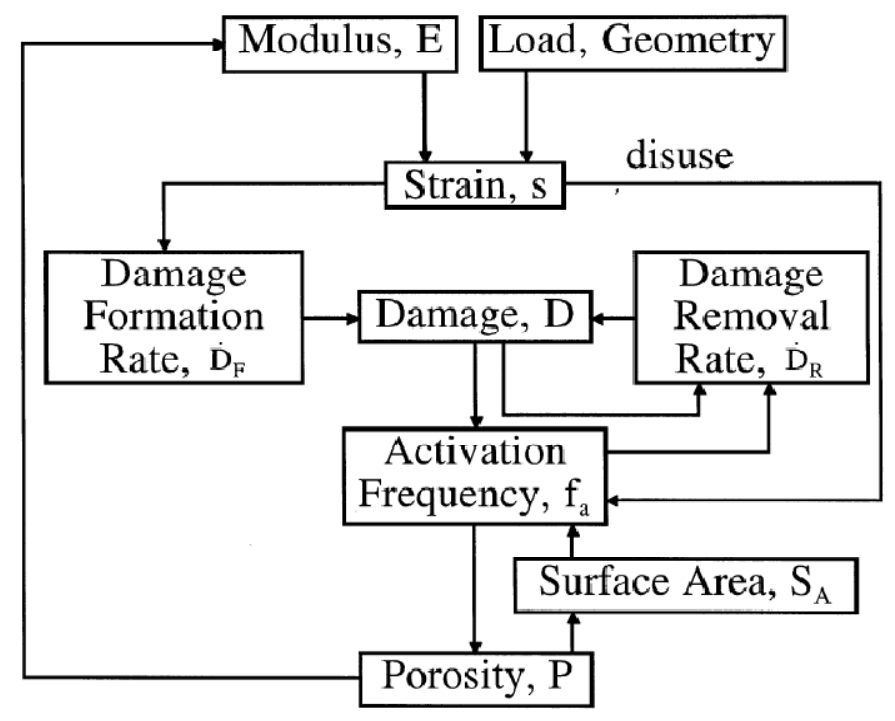

Figure 3-1: Schematic of Hazelwood's damage-repair bone remodeling algorithm. (Hazelwood et al., 2001)

\subsubsection{POROSITY}

Hazelwood's code incorporates the use of a mechanical stimulus and uses the relationship between porosity and elastic modulus to incorporate the material changes produced within the bone for a given loading scenario (i.e. walking, stair climbing). The porosity-modulus relationship is described using a sixth order polynomial (Hazelwood et al., 2001) and assumes a linear relationship between apparent density $(\rho)$ and porosity (p). The porosity rate of change $(\dot{\rho})$ is defined as the difference between the product of the mean bone resorbing rate $\left(Q_{R}\right)$ and the density of resorbing BMUs/area $\left(N_{R}\right)$ and the product of the mean bone formation rate $\left(Q_{F}\right)$ and the density of refilling BMUs/area $\left(N_{F}\right)$ as seen in equation 13 .

$$
\dot{\rho}=Q_{R} N_{R}-Q_{F} N_{F}
$$

where 


$$
\begin{gathered}
Q_{n}=\frac{A}{T_{n}} \\
N_{R}=\int_{t-T_{R}}^{t} f_{a}\left(t^{\prime}\right) d t^{\prime} \\
N_{F}=\int_{t-\left(T_{R}+T_{I}+T_{F}\right)}^{t-\left(T_{2}-T_{I}\right)} f_{a}\left(t^{\prime}\right) d t^{\prime}
\end{gathered}
$$

In the above equations, $A$ is the cross-sectional area of each BMU representing the amount of bone resorbed $\left(\mathrm{mm}^{2}\right), T_{R}$ is the amount of time for the resorption period (days), $T_{I}$ is the amount of time for the reversal period (days), $T_{F}$ is the amount of time for the formation period (days), and $f_{a}$ is the BMU activation frequency (BMUs/area/time). It should be noted that in cortical bone a BMU has an assumed shape of a cylinder with a radius of $.095 \mathrm{~mm}$ (Hazelwood, 2001), whereas in trabecular bone the BMU forms on the boney surface and resorbs a trench. The areas of resorption for either location are assumed to be same. The values of $N_{R}$ and $N_{F}$ are determined by integrating over the appropriate time period (i.e. resorption, reversal, formation) of the BMU activation frequency $\left(f_{a}\right)$ history. As for the values of $T_{R}, T_{I}$, and $T_{F}$, they were determined from multiple histomorphometric studies (Hazelwood et al., 2001).

\subsubsection{ACTIVATION FREQUENCY}

The BMU activation frequency, $f_{a}$, is dependent upon three things: damage, disuse, and internal surface area. The activation frequency represents the amount of BMUs that are recruited to begin the ARF cycle for a particular area for a specified time. Therefore, the activation frequency has units of BMU/area/time. 


\subsubsection{DAMAGE DEFINITION}

Damage $(D)$ can be defined in various ways, but for Hazelwood's model it is the crack length per section area of bone. The damage accretion rate $(\dot{D})$ is then given as the difference between the rate of fatigue damage formation $\left(\dot{D}_{F}\right)$ and the fatigue damage removal $\left(\dot{D}_{R}\right)$. The mechanical stimulus $(\Phi)$ for the model is implemented in defining $\dot{D_{F}}$ and is assumed to be proportional to both the strain range $(s)$ raised to a power and the loading rate $\left(R_{L}\right)$ with units of microstrain and cycles per unit time respectively. The code can accommodate multiple loading conditions simply by summing over the discrete loading conditions, $n$.

$$
\dot{D_{F}}=k_{D} \Phi
$$

where

$$
\Phi=\sum_{i=1}^{n} s^{q} R_{L i}
$$

The variable $k_{D}$ above is called the damage rate coefficient and will be fully defined later in the discussion. As for the $q$ value, it is set at a value of four based upon previous experimental results (Hazelwood et al., 2001). Also, the strain used to compute the mechanical stimulus is assumed to be the principal strain value with the maximum magnitude. It is also assumed that it returns to a zero strain state at the end of each load cycle, allowing the strain range and peak strain to coincide which simplifies the analysis.

The damage removal rate, $\dot{D}_{R}$, is simply the product of $D, f_{a}$, and $A$ if the BMUs and damage are randomly distributed across the bone. However, evidence by multiple authors (Burr et al., 1985, Bentolila et al., 1998) suggests that damage actually initiates BMU activation. This phenomenon is accounted for in the addition of a removal specificity factor $\left(F_{s}\right)$ which increases the efficiency with which damage is removed from the bone. 


$$
\dot{D_{R}}=D f_{A} A F_{S}
$$

With $\dot{D}_{F}$ and $\dot{D}_{R}$ now fully defined, the damage rate coefficient, $k_{D}$, can be determined. Recognizing that in a state of equilibrium $\dot{D}_{F}=\dot{D}_{R}$, the equation can be rewritten and used to determine the damage rate coefficient.

$$
k_{D}=D_{0} f_{a 0} A F_{S} / \Phi_{0}
$$

The subscript 0 in equation 20 denotes the initial, equilibrium values of the variables which are defined at the beginning of the bone remodeling simulation. The equilibrium values are set by the user and in Hazelwood's development were based upon previous averaged data from various authors involving crack length, crack density, and activation frequency in cortical bone to name a few.

\subsubsection{DISUSE DEFINITION}

The remodeling that occurs in response to disuse can be qualified in Hazelwood's model by mechanical stimulus values where $\Phi^{\oplus}<\Phi_{0}$. In disuse situations, less bone is refilled than is removed as bone attempts to optimize its structure via Wolf's law. To account for that fact, the area of bone formed in equation 13 is set to $A\left[\left(0.5+.05\left(\Phi / \Phi_{0}\right)\right]\right.$ (Hazelwood et al., 2001) to effectively reduced the amount of bone replaced by the BMU.

\subsubsection{INTERNAL SURFACE AREA DEFINITION}

Due to the fact that BMUs must begin on a bone surface, the activation frequency, $f_{a}$, then becomes a function of the available boney surfaces. The model does not allow for external bone remodeling (i.e. geometrical changes in bone shape) so the only area of interest is the internal 
surface area. Hazelwood's code uses an empirical formulation (Hazelwood et al., 2001) to normalize the values of internal surface area per unit volume $\left(\mathrm{S}_{\mathrm{A}}\right)$ between zero and one. The formulation is verified for almost any kind of bone (Martin, 1984). The normalized internal surface area is multiplied by the net activation frequency which then allows for a greater potential for remodeling to occur on large surface areas within the bone.

\subsubsection{ACTIVATION FREQUENCY DEFINITION}

Based upon the aforementioned definitions of damage, disuse, and internal surface area, the relationship for $f_{a}$ can be developed. First Hazelwood assumes that $f_{a}$ is a sigmoidal function of both damage and disuse which can be seen in figure 3-2. The coefficients of the equations were based upon curve fitting the proposed functions from previous experimental da
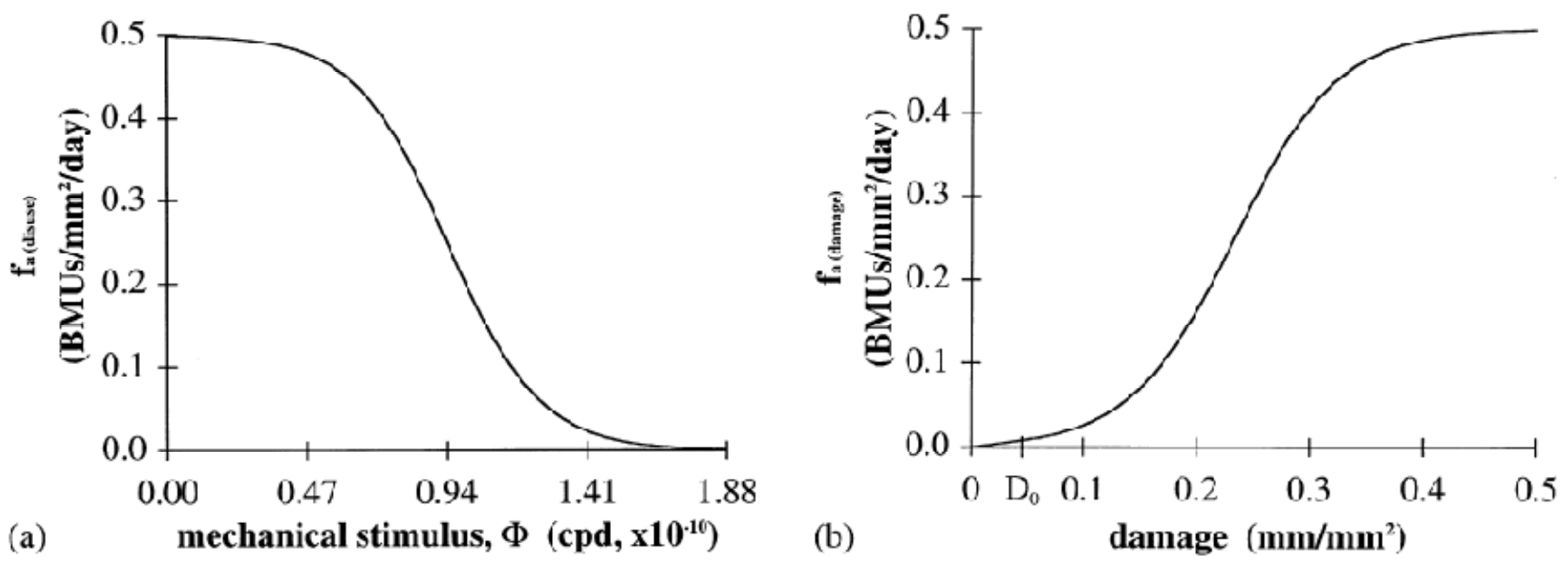

Figure 3-2: Graphical representations describing (a) $f_{a(\text { disuse })}$ as a function of $\Phi$ and (b) $f_{a(\text { damage })}$ as a function of $D$. (Hazelwood et al., 2001)

The equations for the graphs in figure 3-2 are:

$$
f_{a(\text { disuse })}=\frac{f_{a(\max )}}{1+e^{k_{b}\left(\Phi-k_{c}\right)}} \text { for } \Phi<\Phi_{0}
$$




$$
f_{a(\text { damage })}=\frac{\left(f_{a 0}\right)\left(f_{a(\max )}\right)}{f_{a 0}+\left(f_{a(\max )}-f_{a 0}\right) e^{\left[k_{r}\left(f_{a(\max )}\right)^{\left.\left(D-D_{0}\right) / D_{0}\right]}\right.}}
$$

The coefficients $k_{b}$ and $k_{c}$ give the proper shape to the graph in figure 3-2(a) and $f_{a(\max )}$ is defined as the maximum activation energy. In figure 3-2(b), $k_{r}$ defines the shape of the curve and $D_{0}$ represents the initial equilibrium damage value. Finally, with both components of activation frequency fully defined and recalling the internal surface area per unit volume, the overall activation frequency can be described.

$$
f_{a}=\left(f_{\text {a(disuse })}+f_{a(\text { disuse })}\right) S_{A}
$$

\subsection{REMODELING ALGORITHM - VALIDATION}

Ideally, every bone remodeling code would be able to be validated by comparing in-vivo data to the code output. However, quality in-vivo data is difficult to come by due to the invasive nature of obtaining samples as well as the complex loading conditions presented by the soft tissue. As an alternative, Hazelwood's code allows for direct comparison to histomorphometric data. This feature is very powerful and will allow for the possibility that any current study can eventually be confirmed in a future experiment, which is not possible with the other styles of bone remodeling codes discussed in chapter 2. Although Hazelwood's code has not been directly validated via experimental data, a sensitivity analysis of the various system parameters was performed (Hazelwood et al., 2001) and the successful implementation of the code was used to compare various marathon training regimens in femur fracture fatigue analysis (Hazelwood et al., 2007). 


\subsubsection{SENSITIVITY ANALYSIS}

The initial sensitivity analysis performed for Hazelwood's code was based upon the assumptions that daily walking can yield approximately 3,000 cycles per day (cpd) and that an equivalent cyclic strain of $500 \mu \varepsilon$ would create an equilibrium situation for cortical bone per previous results (Hazelwood et al., 2001). For analysis over a 2,000 day period, the cycles were held constant at 3,000 cpd, a compressive force of $891.6 \mathrm{~N}$ (which produced $500 \mu \varepsilon$ ) was applied, and the initial porosity was set to $4.43 \%$ (which allows equilibrium between the Haversion canals created and removed by the BMUs).

The following parameters were varied in order to determine their impact on the model as well as the nature of the various relationships: loading magnitude $(<891 \mathrm{~N}=$ disuse, $>891 \mathrm{~N}=$

overuse), $f_{a(\max )}$ value, the exponential value of $q$ (eq. 18), and time step (ranged from 0.05-8 days). Due to the rapid convergence of the results over the entire range of time steps, a time step of one day was chosen for its convenience in implementation. Figure 3-3 shows the steadystate porosity results over the entire time step range.

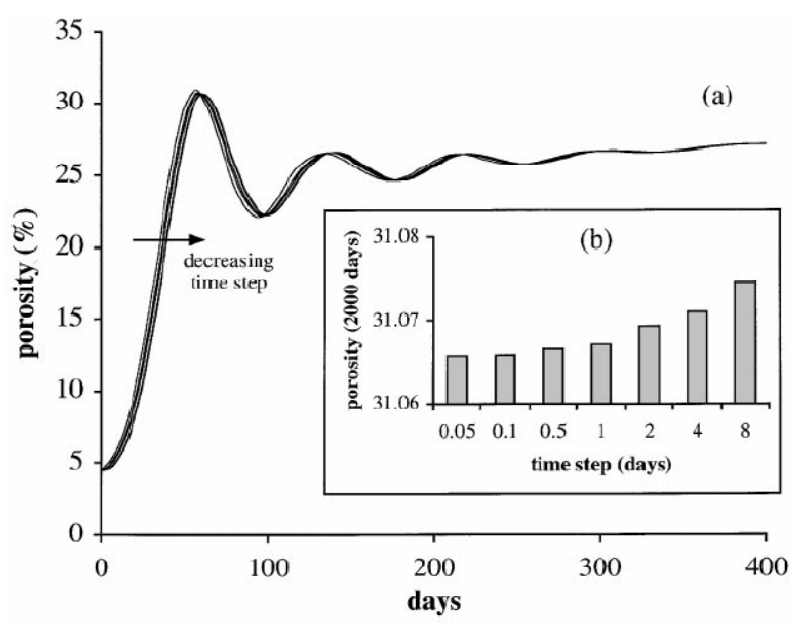

Figure 3-3: Steady state porosity values (a) over an initial 400 day period and (b) at the final 2000 day mark for an initial load of $445.8 \mathrm{~N}$ (time steps ranged from0.05 -8) .(Hazelwood et al., 2001) 
The results were very sensitive to the exponential value $q$ which directly affected the mechanical stimulus. However, the model assumed that the mechanical stimulus is used to determine the degree of disuse as well as the amount of local damage. This assumption simplified the determination of $q$ because multiple authors have created successful models using the same assumption and setting $q=4$ (Hazelwood et al., 2001). Finally the effects of $f_{a(\max )}$ on the porosity and activation frequency were observed and the parameter was set to a value of 1.5. A more extensive view of the sensitivity analysis performed for Hazelwood's code can be found in the actual research paper (Hazelwood et al., 2001).

\subsubsection{FATIGUE ANALYSIS - 2D MODEL}

With the parameters for the model being determined from the sensitivity analysis described above, a 2D application of the remodeling code was then actually run in Abaqus 6.3 in order to compare and contrast various marathon training regimens and how they relate to fatigue analysis. Some of the key features of the model were the assumptions of linear elastic and isotropic material properties along with a constant Poisson's ratio of 0.3 . Also, the initial porosity was set to $4.43 \%$ and the initial baseline mechanical stimulus was set at $\Phi_{0}=1.88 \times 10^{-10}$ cycles per day (cpd), which is needed to maintain bone mass (Hazelwood et al., 2001, Hazelwood et al., 2007).

Three marathon regimens (beginning, medium, advanced) spanning a 16 week period were implemented using Hazelwood's remodeling code to study the effects these had on the femur. Each regimen consisted of weekly mileages as well as rest days with no running. An identical baseline condition for each of the simulations was created using a homogenous set of material properties and allowing the remodeling code to run for 1200 days under normal loading 
conditions. The density results of the baseline model agreed with clinically observed data (Hazelwood et al., 2007) and can be seen in figure 3-4.

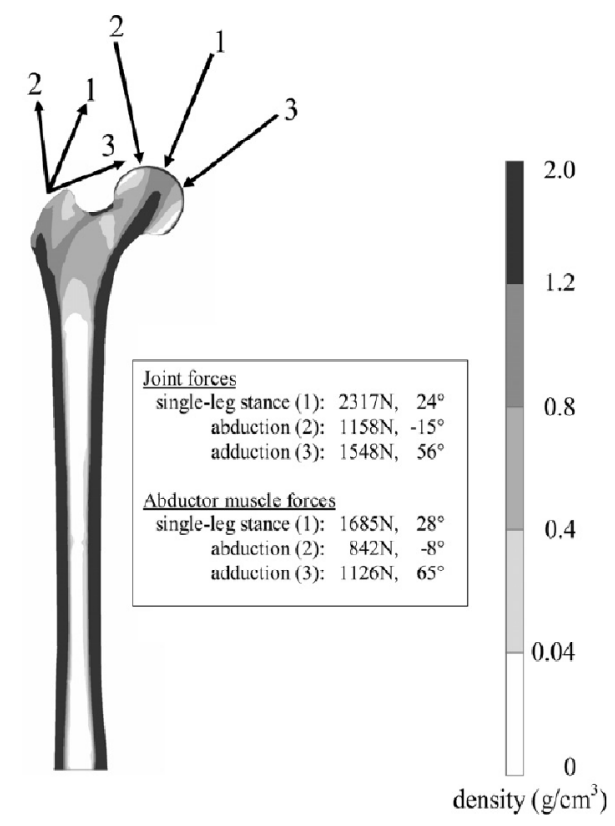

Figure 3-4: Baseline density profile after a 1200 day simulation using Hazelwood's remodeling algorithm to determine a baseline condition for the marathon regimen comparison. (Hazelwood et al., 2007)

Once the baseline was established, the loading conditions of the various training regimens were added to the daily normal activity loads and the simulation was run for the 16 week period. The model predicted an increase in damage over all three training regimens and even trended to having more damage accumulate in the regimens with more miles run as is seen in figure 3-5. 


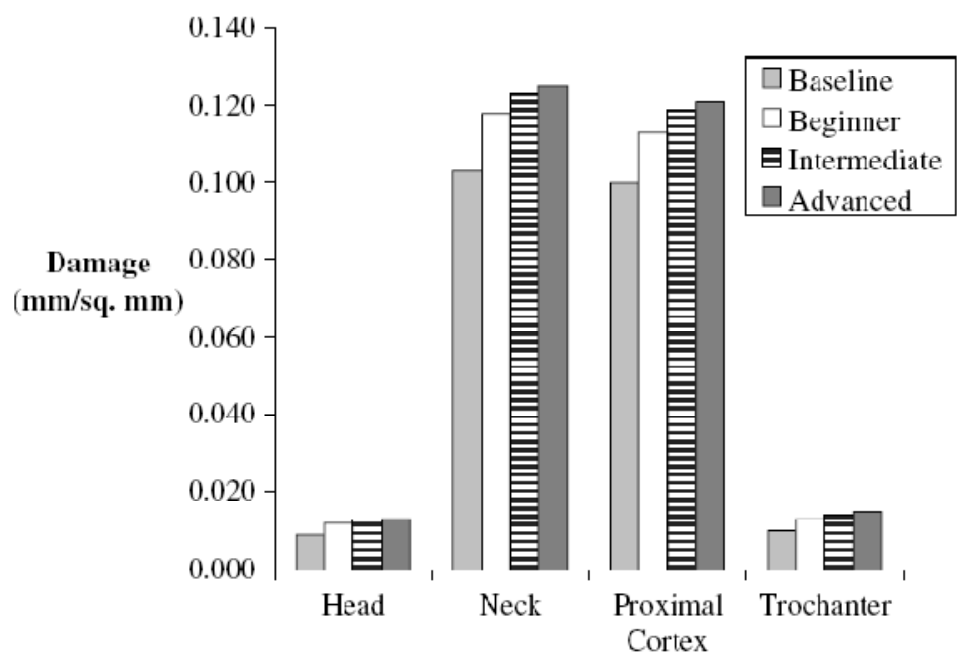

Figure 3-5: Microdamage levels in various regions of interest within the femur over the 16 week period of the various training regimens. (Hazelwood et al., 2007)

Another interesting conclusion from the research was the comparison between two advanced training regimens which were applied over a three year period. The first simulation consisted of the advanced 16 week training program, followed by 3 weeks of rest, and then repeated for three years. Actual marathons were run in each of the three years as well. The second simulation took the same amount of miles accrued as the first simulation but divided it up into daily amounts (recall the advanced regimen had off-days of no running) over an identical pattern - 16 weeks on, three weeks off, and a mix of marathons. The results showed that the damage accrued after three years was predicted to be higher in the daily running scheme compared to the advanced training scheme with rest days; a $14 \%$ increase in the proximal cortex and a $35 \%$ increase in the cortical regions of the femoral neck.

The sensitivity analysis led to a more polished bone remodeling code which was then implemented for damage analysis for marathon training programs (Hazelwood et al., 2007). The results from the damage analysis show how versatile the code is as well as the complex loading regimens it is able to handle. The fact that Hazelwood's code was run with identical loading 
conditions but in different frequencies (e.g. 20 miles a week with three days of rest versus 20 miles a week applied daily) and yielded different damage results is very powerful. Therefore, the Hazelwood code will be implemented into the femur FE model developed in the chapter 4 and will lend credibility to the results obtained over the simulation of the single leg extension.

\subsection{REMODELING ALGORITHM - LEG EXTENSION MODIFICATIONS}

There were only two main changes to the remodeling code proposed by Hazelwood. First, Hazelwood's previous code only incorporated three loading conditions to be applied to the model. For the single leg extension model, four conditions were necessary. This was a minimal change and was easily adapted for the current FE model. The second change was determining how to apply the porosity profile of the subject specific femur to Hazelwood's code. His original code would generate the porosity profile of a femur by applying hip joint and adductor/abductor forces and running the remodeling algorithm. After a certain amount of cycles, the remodeled porosity profile would resemble a typical human femur, with dense cortical bone on the outside edges and around the diaphysis. Since the subject specific femur FE model already had a defined porosity profile associated with it, there needed to be a way to read that data into Hazelwood's code. This was done using a basic Matlab m-file and some text data from subject specific femur. The method will be discussed further in the FE development of chapter 4. 


\section{CHAPTER 4: FE MODEL DEVELOPMENT}

In order to create the most accurate single leg extension FE model, the physical simulation being modeled must be fully described via the computer simulation. In the case of the single leg extension loading, this means including the daily loading due to normal walking activity. If the model did not incorporate the daily loading due to walking, the simulation would assume that the individual did nothing else between the intervals of single leg extension exercises. Therefore, the current study assumed that the individual performs daily walking load cycles in addition to performing the single leg extension exercises.

Based upon the statements above, the final FE model for a single leg extension needs to successfully incorporate walking loads as well as the two loading conditions for a single leg extension, the $105^{\circ}$ and $162^{\circ}$ positions. The creation of the final FE model has numerous steps which are described below:

1. Three Point Bending Validation - this step validates the original material properties of the FE femur with physical experimental data.

2. Loading and BC Application - this step determines the appropriate ways to constrain the femur for each loading configuration as well as how to apply the muscle forces to the FE model.

3. Bone Remodeling: Algorithm - this step modifies the bone remodeling code chosen (Hazelwood et al., 2001) for the single leg extension simulation.

4. Bone Remodeling: Validation - this step validates the modified material properties of the FE femur based upon previous remodeling validation methods (Hazelwood et al., 2001).

5. Final FE Model - this step combines the previous work into one cohesive model. 
The data output from the final FE model will provide information either disproving or proving the hypothesis for the current research - The change in a bone's BMD can be predicted using a modified FE model which incorporates a bone remodeling algorithm.

\subsection{THREE POINT BENDING VALIDATION}

The initial validation of the FE femur is necessary to ensure that assigned material properties are representative of the physical bone. Validation can be achieved in many ways and, typically for FE models, involves recreating a physical test with the model output being compared to the physical test output. Because the main output of concern for the final femur model is strain, it was important to choose a validation method which would easily and effectively allow for strain to be tested. This led to the decision to perform a three point bending test in order to validate the femur FE model.

\subsubsection{THREE POINT BENDING - EXPERIMENTAL SET UP}

A three point bending test was previously performed on the bone specimen during the earlier research in developing the FE femur in ABAQUS (Negrete, 2008). Therefore, a lot of the experimental preparation was already done (Negrete, 2008). The strain gauges used previously were permanently applied to the bone and were found to be applied approximately in the direction of the principal axes on the anterior/posterior surface of the bone (Negrete, 2008). This allowed for the three point bending test set up to utilize the previously placed gauges with the knowledge that they were oriented in a fashion which would allow the strain outputs to be easily compared to the ABAQUS FE model (e.g. ABAQUS has Visualization options which allow the user to output strain in the principle directions, EE1/EE2/EE33). During the time between the three point bending experiment performed by Negrete and the experiment for the current body of 
work, the bone was wrapped in gauze and stored in a freezer. When it was time to perform the experiment, the bone was removed from the freezer and was allowed to thaw for a full 24 hours, which was identical to the previous experimental preparation (Negrete, 2008). The orientation of the bone in the Instron seen in figure 4-1 was attempted to be the same as for Negrete's experimental orientation.

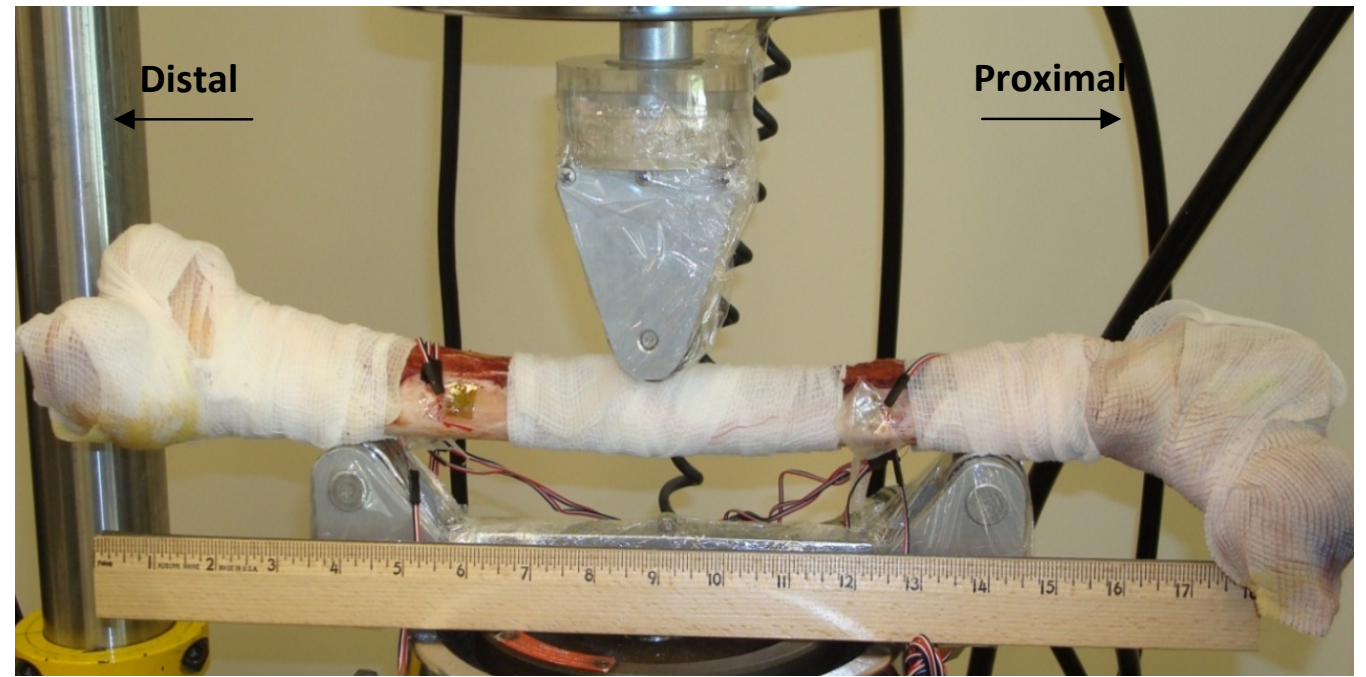

Figure 4-1: Experimental Three Point Bending set up in an Instron with attached strain gauges.

However, due to the fact that the diaphysis of the femur is more triangular than circular, the femur was not able to be placed in such a manner so as to have the cross-head of the Instron compress the bone in a posterior to anterior direction. The natural position of the bone can be seen in figure 4-1 where it is observed that the femoral head and condyles are rotated such that the most posterior surface of the bone is not in contact with the cross-head of the Instron. This is important to note because the proper orientation must be duplicated in the ABAQUS model in order to obtain valid data. It should also be noted that two strain gauges on the anterior surface of the femur were used for the validation process (labeled 'Rosette B' and 'Rosette D'). A 
graphical representation of the location of the strain gauges on the anterior surface can be seen in

Figure 4-2.

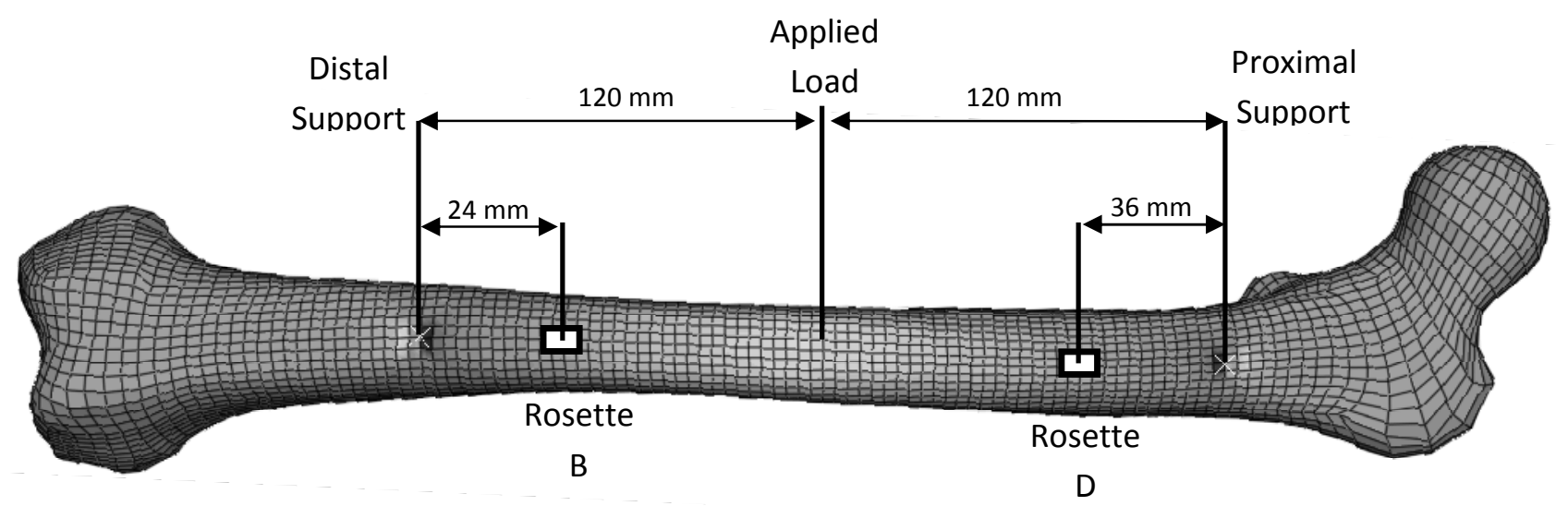

Figure 4-2: Anterior View of the femur with the approximate locations of strain gauge Rosette $B$ and strain gauge Rosette D. The distal/proximal supports and applied load are shown for reference.

\subsubsection{THREE POINT BENDING - DATA ACQUISITION}

A displacement controlled test profile for the Instron was chosen for the experimental test. The profile incrementally displaced the cross-head at a constant rate until the specified total displacement was reached. For the three point bending profile performed, the cross-head displaced at a rate of $.10 \mathrm{~mm} / \mathrm{s}(-0.004 \mathrm{in} / \mathrm{s})$ to a final displacement of $2.9 \mathrm{~mm}(0.11 \mathrm{in})$. The displacement and strain outputs for the test were continuously recorded via data acquisition software. The Instron output the displacement of the cross-head as well as the reaction force at the cross-head as a function of time. The strain gauges output the principal strain values in the longitudinal axes of the diaphysis of the bone over time. In this way, the displacement-strain and force-strain plots could be created in order to directly compare to the results from the FE femur three point bending test. 
The three point bending experiment was run a total 10 times. The first run was a trial test in order to make sure that all of the equipment was working properly. Experimental runs two through six involved the Instron displacing the bone the required $2.9 \mathrm{~mm}$. The remaining four runs increased the maximum displacement of the Instron cross-head in 1mm increments until the bone fractured.

\subsubsection{THREE POINT BENDING - FE MODEL}

Using the femur FE model previously developed (Negrete, 2008) and the information provided by figures 4-1 and 4-2, an ABAQUS model of the three point bending test was created. The proximal and distal supports were modeled as rigid bodies and were constrained to the bone via tie constraints. The cross-head displacement was applied to a single node which was manually displaced to a minimal value of $2.9 \mathrm{~mm}$. The ABAQUS model performed a quasistatic analysis over the course of 12 steps by displacing the single node $.23 \mathrm{~mm}$ per step. Although the model could have been run in one step due to it being in the linear-elastic range of the material properties of the bone, it was run in such a manner so as to be completely analogous to the experiment. The results compared to the physical experiment were the strain values at each of the two rosettes as well as the overall load required to produce the $2.9 \mathrm{~mm}$ of deflection.

\subsubsection{THREE POINT BENDING - VALIDATION RESULTS}

The results of the ABAQUS FE model for three point bending were directly compared to the data collected during the physical experiment. The force-deflection and strain-deflection plots are compared in figures 4-3 and 4-4 and show the close agreement between the FE model and the experiment (less than $1 \%$ difference). 


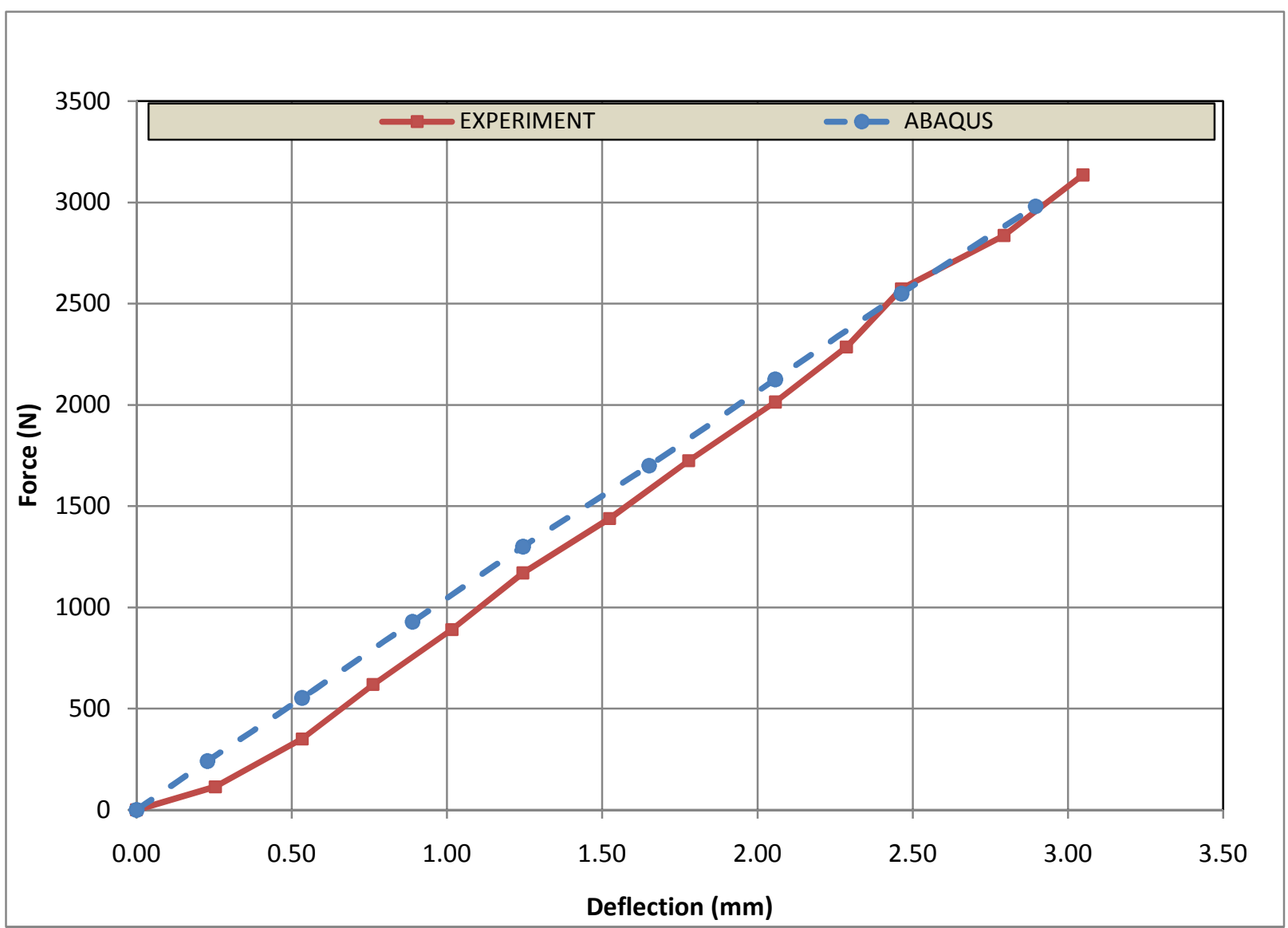

Figure 4-3: Force-deflection comparisons between the three point bending experiment and the FE model. 


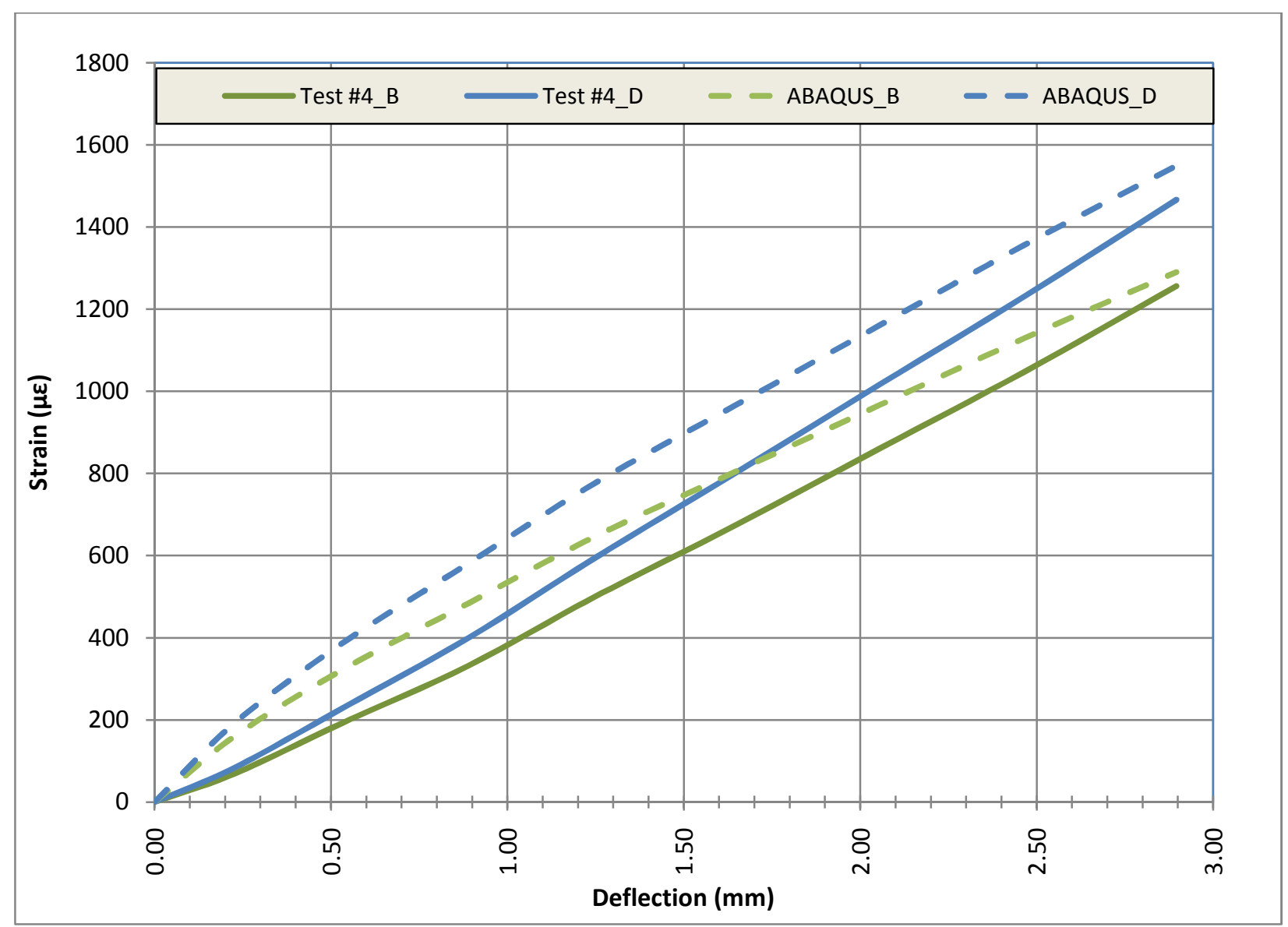

Figure 4-4: Strain-deflection comparisons between the three point bending experiment and the FE model for test run \#4.

The FE model strain results were obtained by averaging nodal strain values over a surface area determined to be equivalent to the surface area covered by the physical gauges, approximately $3 \mathrm{~mm} \times 7 \mathrm{~mm}$. In the FE model, this consisted of six nodal values for each of the rosettes on the bone. The locations of the six nodes representing the rosettes were determined using the known location of the rosettes on the actual bone as well as the orientation of the test per figure 4-1. Using the nodal principal strain values from the output database file generated by ABAQUS, the FE model predicted strain values were able to be averaged and determined at each rosette location. The physical experimental data for each strain rosette can be seen in figure $4-5$. The 
averaged FE model strain values of $1290 \mu \varepsilon$ (rosette B) and $1550 \mu \varepsilon$ (rosette D) agree well with the average experimental values for rosettes B and D, $1250 \mu \varepsilon$ and $1460 \mu \varepsilon$ respectively.

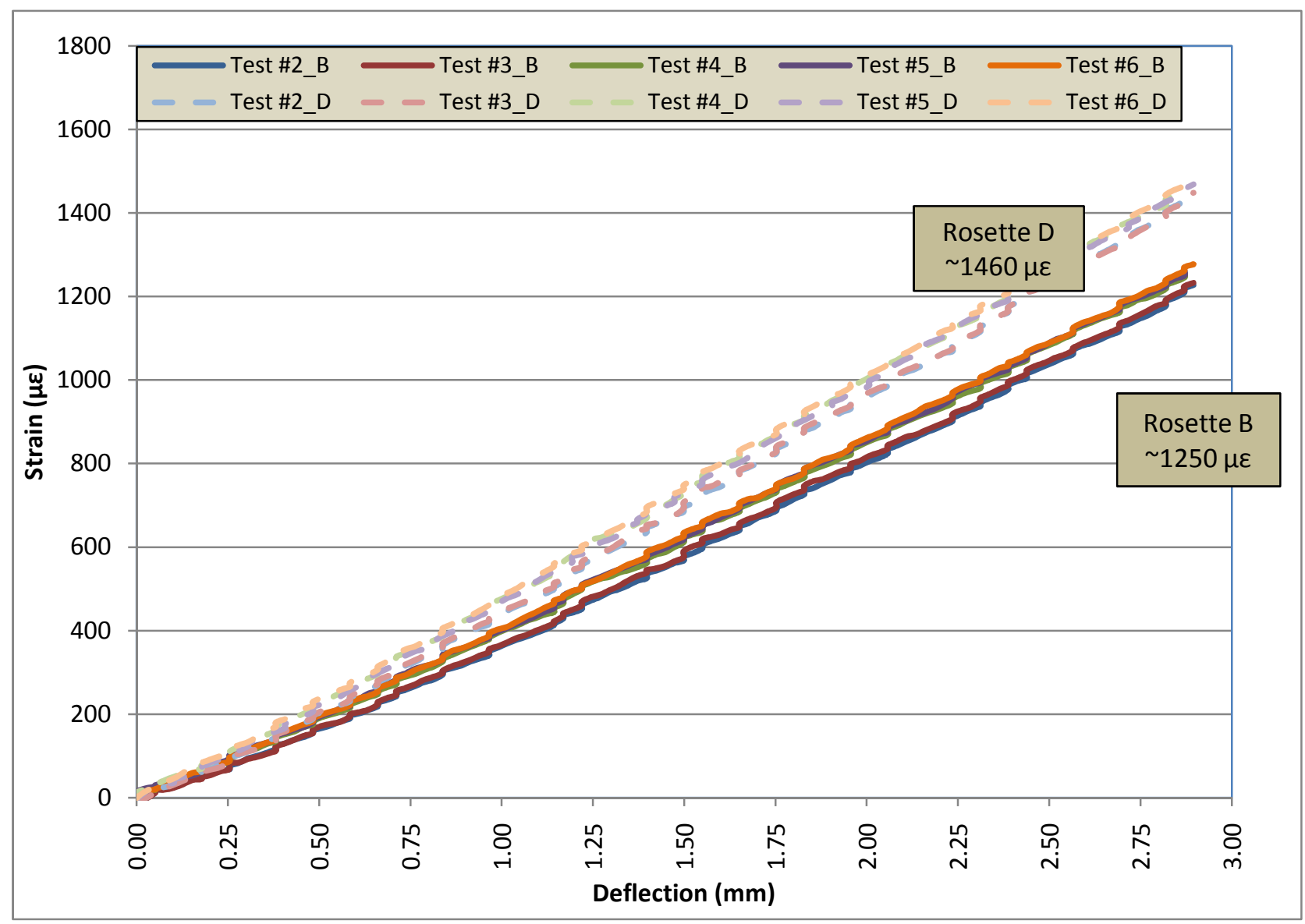

Figure 4-5: Three point bending strain rosette $B$ and $D$ values for experimental test runs \#2 through \#6.

The close agreement between the physical experiment and the FE model concludes the material property validation for the FE femur created in ABAQUS. The FE model applied load and strain values were all within $6 \%$ of the experimentally determined values. The material property validation of the FE model is crucial in creating a realistic FE model of a single leg extension. The next step in the development process is the application of the muscle loads and boundary conditions for a single leg extension. 


\subsection{LOADING \& BOUNDARY CONDITION APPLICATION}

The definition of the proper BCs and loading for a single leg extension can greatly affect the outcome of the model. Both over- and under-constraining an FE model can lead to nonphysiologic results. Based upon the previous research (section $2.2 \& 2.3$ ) it was determined that the joint constraint BCs along with muscle forces applied over multiple nodes would create the most physiologic results. However, within ABAQUS there are various ways to apply the distributed muscle forces over a particular area. Also, the surface area of the various muscles can affect how the force is distributed; for example, the Vastus Intermedius covers an area approximately $1 / 3$ of the total length of the femur that wraps from the anterior surface around to the posterior surface on the lateral side. A comparative study between various muscle attachment site sizes and methods of attachment was performed in order to see how the strain results of the FE model would be affected, if at all.

Once the results of the comparative FE study performed in ABAQUS have been discussed, the individual loading and $\mathrm{BCs}$ for each of the loading conditions for the final FE model will be specified. The loading conditions include daily walking, the $105^{\circ}$ single leg extension, and the $162^{\circ}$ single leg extension.

\subsubsection{LOADING \& BC - FE COMPARISON}

A set of three comparative FE models were created to determine the most appropriate way to apply the muscle forces. The forces applied to the models are representative of a $105^{\circ}$ single leg extension. Table 4-1 below contains the applied loads for the FE model that was used to compare the three muscle application cases. 
Table 4-1: Applied $105^{\circ}$ single leg extension loads of the FE model used to compare three methods of muscle attachment.

\begin{tabular}{|c|c|c|c|c|c|}
\hline \multicolumn{7}{|c|}{ FE APPLIED LOADS } \\
\hline & V. LAT. & V. INT. & V. MED. & Condyle & Patella \\
\hline x-component & -25.5 & -3.5 & -29.1 & 0 & 138.4 \\
\hline y-component & -119.8 & -20.2 & -33 & 126.5 & 874 \\
\hline z-component & 11.1 & 0 & 33.2 & 371 & -690 \\
\hline OVERALL MAGNITUDE & $\mathbf{1 2 3}$ & $\mathbf{2 1}$ & $\mathbf{5 5}$ & $\mathbf{3 9 2}$ & $\mathbf{1 1 2 2}$ \\
\hline \multicolumn{7}{|c|}{} \\
\hline
\end{tabular}

Each of the three model cases is described in detail below.

- Small Patch, Reference Point (SPRP) - A small patch is used to represent the approximate muscle attachment site. A surface vector is created using a reference point (RP) located at the center of the muscle patch. The coupling constraint in ABAQUS is used to connect the RP to the muscle patch. The force vector is applied directly to the RP.

- Small Patch, Nodal Traction (SPNT) - Identical muscle patch size and location as Case 1. The force vector is divided amongst the number of nodes in the muscle patch and applied directly to the individual nodes.

- Large Patch, Reference Point (LPRP) - A muscle patch that is representative of the complete attachment site is created using multiple small patches (this only affects the Vastus Intermedius). The force vector is divided by the number of small patches and applied to the various RPs which are constrained to the bone via the coupling constraint. 
All three FE models were bounded by way of the joint boundary condition (Speirs et al., 2006).

Recall from section 2.2, that the joint BC incorporates a hip constraint as well as a constraint on the lateral condyle. The degrees of freedom (DOF) that are applied to the FE model for the joint BC can be seen in table 4-2.

Table 4-2: DOF associated with the Joint BC in ABAQUS FE models

\begin{tabular}{|c|c|c|c|c|c|c|}
\hline & U1 (x) & U2 (y) & U3 (z) & UR1 (x) & UR2 (y) & UR3 (z) \\
\hline HIP JOINT & $\mathrm{x}$ & $\mathrm{x}$ & $\mathrm{x}$ & $\mathrm{x}$ & $\mathrm{x}$ & $\mathrm{x}$ \\
\hline LAT. CONDYLE & $\mathrm{x}$ & free & $\mathrm{x}$ & $\mathrm{x}$ & $\mathrm{x}$ & $\mathrm{x}$ \\
\hline
\end{tabular}

Each of the three cases was run in ABAQUS. The reaction forces as well as the strain magnitudes and distributions were used for comparison. Table 4-3 contains the data used to compare the three modeled cases and figure 4-6 highlights the various strain distributions on the anterior surface of the femur.

Table 4-3: SPRP, SPNT, \& LPRP hip, lateral condyle, and strain data.

\begin{tabular}{|c|c|c|c|}
\hline & HIP RXN (N) & LAT. COND. RXN (N) & MAX. STRAIN ( $\boldsymbol{\mu \varepsilon})$ \\
\hline SPRP & 394 & 60 & 8407 \\
\hline SPNT & 394 & 62 & 8499 \\
\hline LPRP & 393 & 65 & 7149 \\
\hline
\end{tabular}



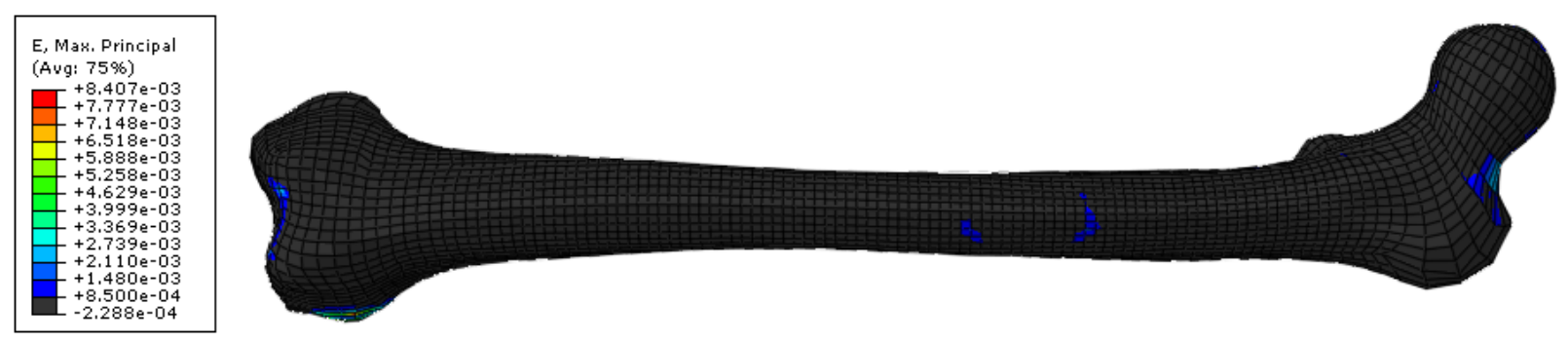

(a) SPRP strain distribution for a $105^{\circ}$ single leg extension exercise.
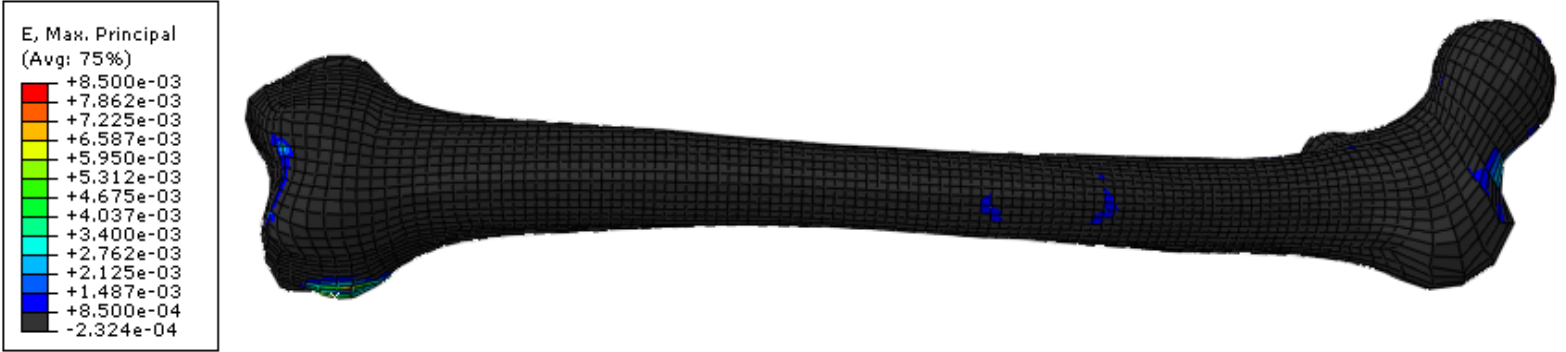

(b) SPNT strain distribution for a $105^{\circ}$ single leg extension exercise.
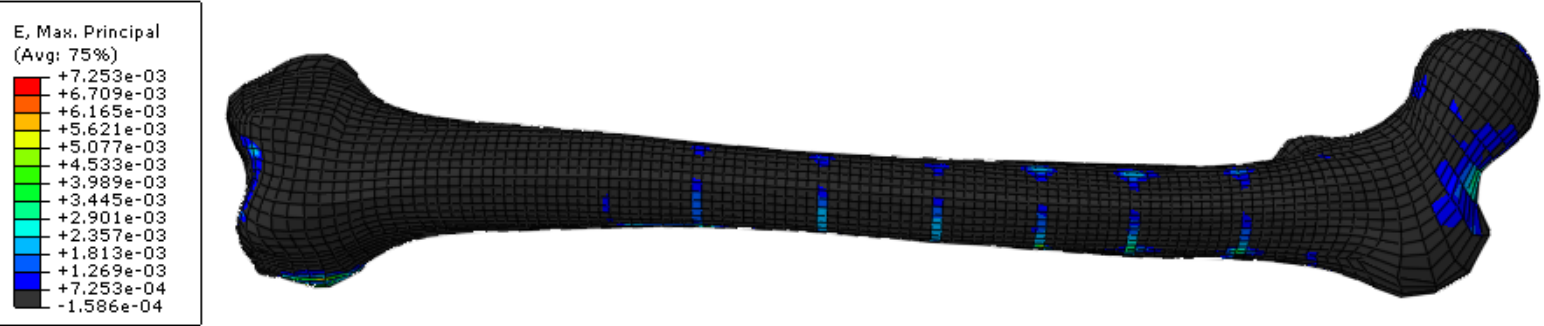

(c) SPRP strain distribution for a $105^{\circ}$ single leg extension exercise.

Figure 4-6: Anterior view of $\mathrm{FE}$ femur model with the maximum strain distributions for each of the three model case, (a) - (c). Strain range: $0.008500 \varepsilon-0.000500 \varepsilon$.

Based upon the fact that each of the three cases had equivalent load magnitudes and directions, it was reasonable that the hip and lateral condyle reaction forces were similar in all three cases. As for the maximum strain magnitude and distribution, both small patch models exhibited similar values and were essentially interchangeable methods. The results for the large patch model agreed with previous observations of a more distributed force yielding lower maximum strain values over the bone (Polgar et al., 2003). However, the implementation of a more distributed 
vastus intermedius muscle force in ABAQUS actually created non-physical peak strain "bands" which can be seen in figure 4-6 (c).

Although the artificial "bands" could be removed if the loads in the large patch model were applied to every individual node (identical to the SPNT), the LPRP model would still not have been considered a viable model for the final implementation of muscle forces for a single leg extension exercise. There were still too many unknowns in the muscle-bone interaction which would make the force distribution over a large attachment area difficult to model well (e.g. should the force magnitude be evenly distributed?, does the distributed force change directions depending on location?) Even though the same questions could be asked in the small patch models, previous research performed on walking simulations have had great success with small patches of distributed forces (Duda et al., 1998, Stolk et al., 2001, Speirs et al., 2006,). Therefore, based upon previous research and the comparative study performed above, the final FE model for a single leg extension applied the muscle forces in a small patch manner. Because each small patch model produced similar results, the final FE model used the SPRP method due to it being less time-consuming and easier to implement than the SPNT model.

\subsubsection{LOADING \& BC - WALKING}

Although numerous authors have documented the required muscles and BCs used to simulate walking (Cristofolini et al., 1995, Duda et al., 1998, Stolk et al., 2001, Speirs et al., 2006), the most applicable model involved two separate phases of walking - heel strike, heel strike - in order to capture the daily loading due to walking (Deuel, 2007). Deuel's model included the following muscles: gluteus medius, gluteus minimus, psoas, vastus lateralis, adductor brevis, adductor longus. The hip joint force was modeled as a distributed pressure over the appropriate area of the femoral head. A fully constrained set of nodes at the distal diaphysis of the femur 
was used to constrain the model. Because the walking loads and BCs for Deuel's FE femur model have successfully been implemented in a FE code (Deuel, 2007), the force magnitudes, directions, and BCs for the heel strike and toe off phases of walking of his model were directly applied to the single leg extension FE model. The applied walking loads and BCs for each model can be seen in figure 4-7.
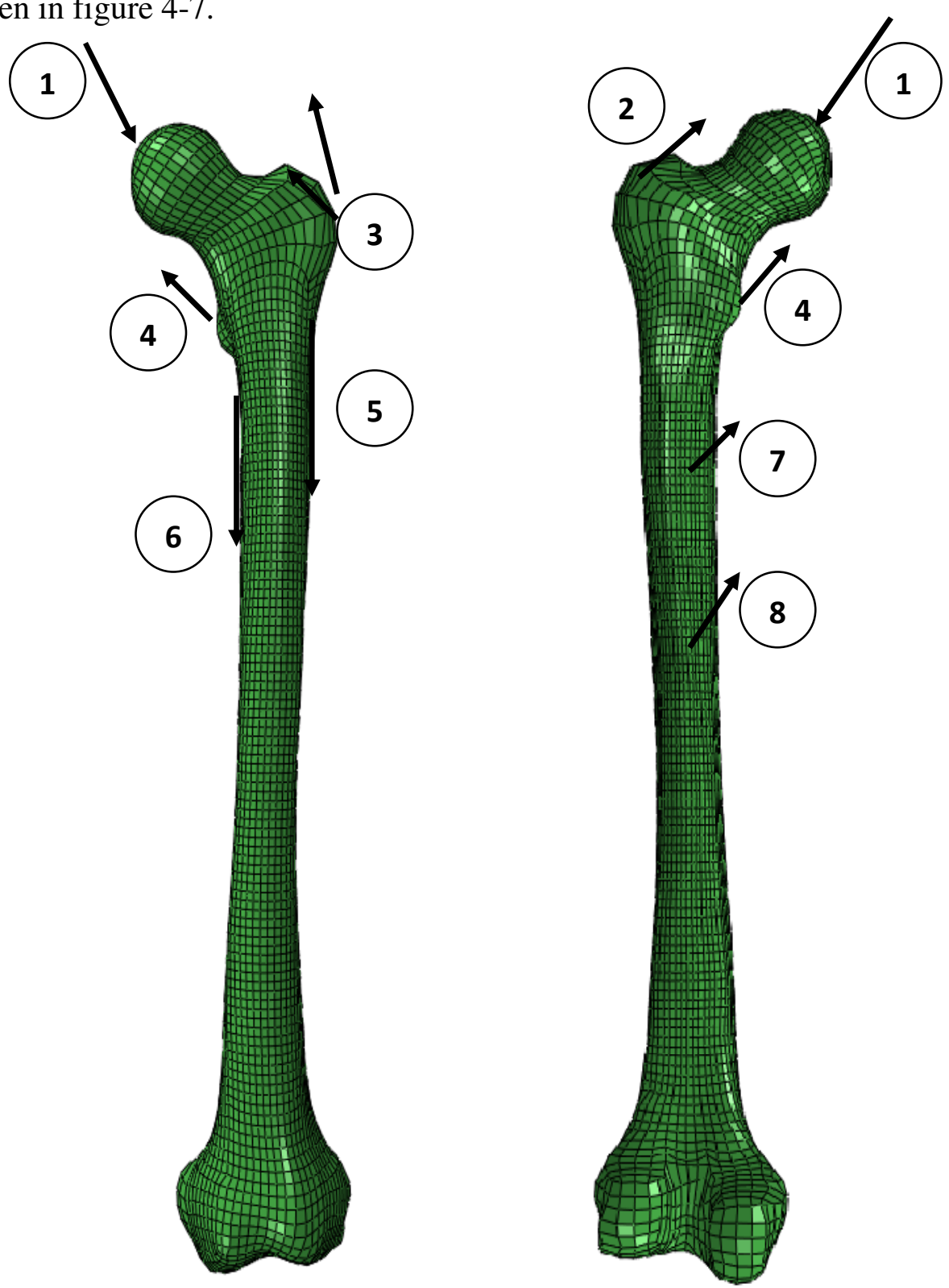

Figure 4-7: Applied walking muscles/BC for the single leg extension $\mathrm{FE}$ model in the (a) anterior and (b) posterior views: (1) Hip Joint, (2) Gluteus Medius, (3) Gluteus Minimus, (4) Psoas, (5) Vastus Medialis, (6) Vastus Lateralis, (7) Adductor Brevis, (8) Adductor Longus. 
All muscles were applied to the bone via a SPRP except for the adductor brevis and longus. Due to the fact that they are so thin and long, their muscle magnitudes were distributed evenly among individual nodes. Also, because the single leg extension femur FE model actually had the condyles modeled, whereas Deule's FE femur did not, three separate nodes were constrained at the condyles to fully confine the model, as seen in figure 4-7 (d). The walking forces which were applied to the single leg extension femur model for both toe on and heel off can be seen in table 4-4.

Table 4-4: Muscle force magnitudes for 3D loads involving the toe on and heel off phases of walking.

\begin{tabular}{|c|c|c|c|}
\hline & Walking Loads_Phase I \\
\hline & $\mathbf{X}$ & $\mathbf{y}$ & $\mathbf{z}$ \\
\hline Adductor Brevis & -1.15 & 1.28 & -0.07 \\
\hline Adductor Longus & -2.08 & 3.27 & -1.70 \\
\hline Gluteus Minimus & -148.53 & 327.43 & 125.71 \\
\hline Gluteus Medius & -297.16 & 664.30 & 251.70 \\
\hline Vastus Lateralis & -132.91 & -677.46 & 103.64 \\
\hline Hip & \multicolumn{3}{|c|}{ 2,052 distributed load } \\
\hline \multicolumn{4}{|c|}{ Walking Loads Phase II } \\
\hline Adductor Brevis & -1.15 & $\mathbf{y}$ & $\mathbf{z}$ \\
\hline Adductor Longus & -2.08 & 3.27 & -0.07 \\
\hline Gluteus Minimus & -72.00 & 344.64 & -1.70 \\
\hline Gluteus Medius & -156.28 & 728.98 & -120.97 \\
\hline Psoas & -54.70 & 147.8 & 70.71 \\
\hline Hip & 2,052 distributed load $^{*}$ \\
\hline
\end{tabular}

\subsubsection{LOADING \& BC - SINGLE LEG EXTENSION, $105^{\circ}$}

The BCs for the single leg extension were the joint BCs proposed by Speirs et al. (Speirs et al., 2006) and the muscle forces were applied via the SPRP method. The magnitude, direction, and location of the applied muscles will be discussed in further detail for the final single leg extension FE model. 
The joint and muscle forces for the $105^{\circ}$ extension were calculated by a specialized biomechanical simulation. Muscle EMG data, bone geometry, and statics were used to determine the various forces (Scott, 2008). For simplicity, a two-dimensional (2D) analysis was performed using a reduced muscle set that represents approximately $90 \%$ of the active muscles during a leg extension - vastus medialis, vastus lateralis, vastus intermedius, and rectus femoris. Due to the data collection method of EMG data, only surface muscles were able to be recorded. Therefore, the biomechanical simulation did not directly record data for the vastus intermedius, although it was accounted for by scaling the other muscles in the leg to account for the lack of it (Scott, 2008). In order to ensure that the simulated loads were properly applied to the single leg extension FE model three steps were required: (1) determination of the proper three-dimensional (3D) muscle locations (2) redistribution of the appropriate muscle forces (3) verification that the forces balance in ABAQUS.

\subsubsection{1. $105^{\circ}$ Extension: 3D Femur Muscle Locations}

Before muscle location data for various bones was readily available, researchers used Gray's Anatomy to approximate the location. However, even with published muscle attachment data for the femur (Brand et al., 1982) it was noted that a general data set would be very difficult to create because muscle locations can vary so much between individuals. Therefore, determining the final muscle locations for the single leg FE femur was an iterative process that involved implementing data from various authors until the final model looked reasonable. The final muscle configuration was based upon a muscle set used by Cristofolini et al. in a paper for gait analysis (Cristofolini, 1995) and is shown in figure 4-8. 


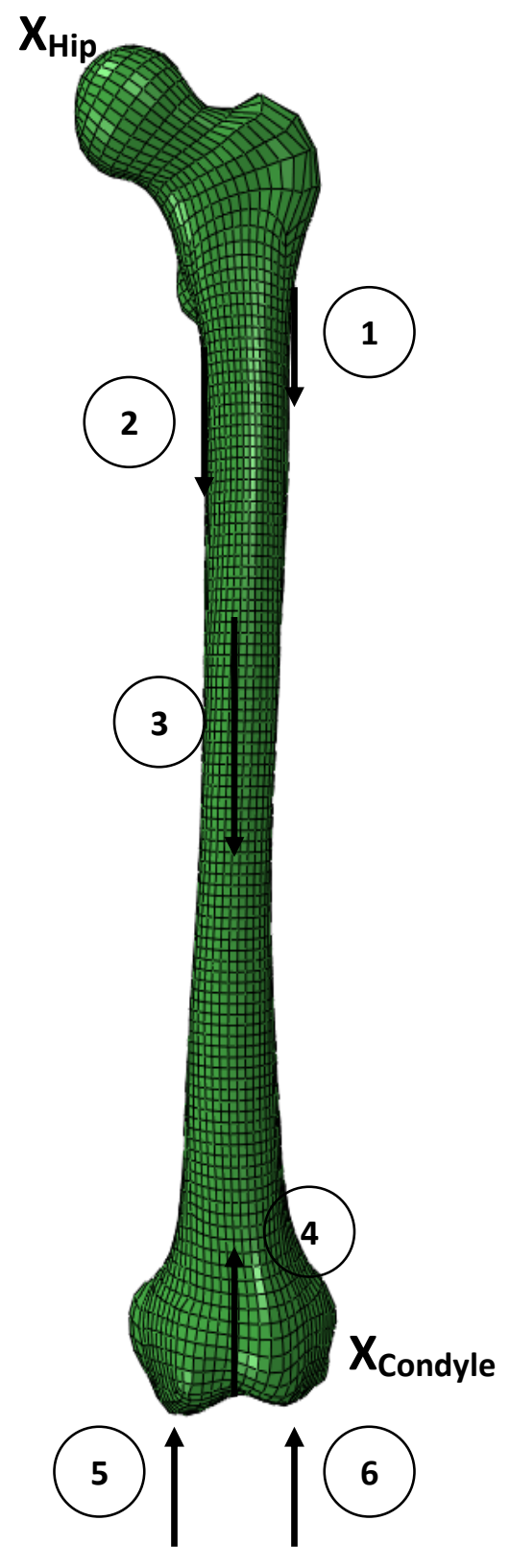

Figure 4-8: Force and BC locations for single leg extension FE model: (1) Vastus Lateralis, (2) Vastus Medialis, (3) Vastus Intermedius, (4) Patella Contact, (5) Medial Condyle Contact, (6) Lateral Condyle Contact, Hip Constraint $\left(\mathbf{X}_{\text {hip }}\right)$, and Lateral Condyle Constraint $\left(\mathbf{X}_{\text {condyle }}\right)$.

\subsubsection{2. $105^{\circ}$ Extension: Muscle Force Distribution}

The 2D forces determined by the biomechanical simulation (Scott, 2008) needed to be redistributed in order to effectively model a 3D single leg extension. Recall that the EMG data 
was only able to collect data for surface muscles and that the vastus intermedius is a subsurface muscle, located posteriorly to the rectus femoris. The simulation which determined the muscle forces accounted for this lack of a muscle force by scaling up the other two active muscles, namely the vastus lateralis and vastus intermedius. The vastus intermedius is critical in creating a balanced, physiologic model of the single leg extension and therefore its force needed to be included for the final FE femur analysis.

The redistribution of forces only affected the predicted muscle magnitudes of the biomechanical simulation; therefore, the previously predicted hip, patella, and condyle forces were unaffected. The redistribution of forces was based upon the physiologic cross-sectional area (PSCA) for each of the muscles as well as the measured force output collected from the EMG data. The full details of the force redistribution can be seen in Appendix A. Table 4-5 summarizes the modified muscle force magnitudes as well as the joint reaction forces for the $105^{\circ}$ leg extension.

Table 4-5: Muscle force magnitudes for a $3 D 105^{\circ}$ single leg extension.

\begin{tabular}{|c|c|c|c|c|}
\hline Muscle & x-component (N) & y-component (N) & z-component (N) & Magnitude (N) \\
\hline Vastus Medialis & -29.1 & -333 & 33.2 & 336 \\
\hline Vastus Lateralis & -25.5 & -119.8 & 11.1 & 123 \\
\hline Vastus Intermedius & -44.7 & -282.2 & 0 & 286 \\
\hline Joint Location & \multicolumn{5}{|l|}{} \\
\hline Hip & -11.8 & -392 & -42.6 & 395 \\
\hline Patella & 138.4 & 874 & -690 & 1122 \\
\hline Condyles* & 0 & 126.5 & 371 & 392 \\
\hline
\end{tabular}

*The condyle load was applied per condyle in the 3D model.

\subsubsection{3. $105^{\circ}$ Extension: Validate Muscle Force Equilibrium}

The last step for simulating the $105^{\circ}$ leg extension loads was to validate that the $3 \mathrm{D}$ reactions were still similar to the original predicted $2 \mathrm{D}$ reactions. The muscle, condyle, and patella forces were applied directly to a FE femur with joint BCs. A static analysis in ABAQUS was used to 
predict the resultant hip force. The ABAQUS predicted hip force was then compared to the hip force predicted by the biomechanical simulation. The FE model was considered validated if the hip force magnitude output was within $\pm 5 \%$ of the predicted hip force magnitude.

For the FE femur model, the joint $\mathrm{BC}$ was made up of one node set at the femoral head and another at the lateral condyle. Both node sets were implemented via rigid body connections in ABAQUS. The node patch at the femoral head was fully constrained while the node patch at the lateral condyle was only allowed to translate in the proximal-distal directions (i.e. no translation was permitted in the medial-lateral or anterior-posterior directions and no rotation was allowed about any coordinate axis).

Due to the fact that the loading for the 2D simulation was being applied to a 3D FE femur model, additional reactions were required to balance the system. The additional forces required to keep the system in a state of static equilibrium were added in the form of a lateral condyle constraint. In-vivo these additional forces would be resisted by the soft tissue ligaments in the knee. A study of knee ligament strength (Kennedy et al., 1976) determined that the posterior cruciate ligament, tibial collateral ligament, and the anterior cruciate ligament could individually support a maximum load of $460 \mathrm{~N}$. However, seeing as how the additional forces are a result of an incomplete set of forces (i.e. full muscle, tendon, and ligament data) the lateral condyle reaction force should be kept to a fraction of the applied loads in order to be deemed acceptable. Therefore, the reaction force at the lateral condyle should not exceed $20 \%$ of the maximum load applied and should be no larger than the maximum load for a single knee ligament. For the $105^{\circ}$ leg extension exercise, the maximum applied load was $1122 \mathrm{~N}$. This meant that the lateral condyle reaction magnitude must be below $224 \mathrm{~N}$ in order to satisfy the criteria above. The results can be seen in Table 4-6. 
Table 4-6: $105^{\circ}$ extension validation results: $\mathrm{P}=$ Predicted Force, $\mathrm{A}=\mathrm{ABAQUS}$ Force

\begin{tabular}{|c|c|c|c|c|}
\hline Constraint Type & x-comp. (N) & y-comp. $(\mathbf{N})$ & z-comp. $(\mathbf{N})$ & Magnitude (N) \\
\hline Hip Joint (P) & n/a & -392 & -127 & 412 \\
\hline \multicolumn{5}{|l|}{} \\
\hline Hip Joint (A) & -11.8 & -392 & -42.6 & 395 \\
\hline Lateral Condyle (A) & -27.3 & 0 & -53.7 & 60 \\
\hline
\end{tabular}

The results showed that the lateral condyle force and the hip reaction force both met the determined criteria. Although the ABAQUS hip reaction force magnitude was within 5\% of the predicted hip joint magnitude, there was a large discrepancy between the two z-components. That observation led to the discovery that the original 2-D biomechanical simulation results were not in complete static equilibrium. Since a quasi static analysis method will be used for the final single leg extension FE model, the applied forces must be in static equilibrium. The forces predicted from the $105^{\circ}$ ABAQUS model were in static equilibrium and yielded valid hip and condyle force magnitudes. Therefore, it was deemed acceptable to use the ABAQUS loads presented in table $4-6$ for the $105^{\circ}$ single leg extension.

\subsubsection{LOADING \& BC - SINGLE LEG EXTENSION, $162^{\circ}$}

The $162^{\circ}$ extension load case was determined in the same fashion as the $105^{\circ}$ load case. The joint BCs (Speirs et al., 2006) were applied to the femur and the SPRP method was used for the muscle loading. The $2 \mathrm{D}$ forces for the $162^{\circ}$ single leg extension also needed to be redistributed and required the following steps: (1) redistribution of the appropriate muscle forces (2) verification that the forces balance in ABAQUS. The determination of the $3 \mathrm{D}$ muscle locations was not necessary because it was previously performed for the $105^{\circ}$ single leg extension model. 


\subsubsection{1. $162^{\circ}$ Extension: Muscle Force Distribution}

The muscle distribution for the $162^{\circ}$ leg extension FE model was identical to the method used for the $105^{\circ}$ extension with one exception. The EMG sensor used to predict the muscle force for the vastus lateralis malfunctioned and the data had to be discarded. In order to determine the loading distribution, the assumption that the activation energy for each of the three muscles is constant throughout the single leg extension exercise was made. The details of the muscle force distribution can be seen in Appendix A and the results are shown in Table 4-5.

Table 4-7: Muscle force magnitudes for a 3D $162^{\circ}$ single leg extension.

\begin{tabular}{|c|c|c|c|c|}
\hline Muscle & x-component (N) & y-component (N) & z-component (N) & Magnitude (N) \\
\hline Vastus Medialis & -5.8 & -66.1 & 6.6 & 67 \\
\hline Vastus Lateralis & -5.1 & -23.8 & 2.2 & 24 \\
\hline Vastus Intermedius & -8.9 & -56.1 & 0 & 57 \\
\hline Joint Location & -4.6 & -123 & -16.3 & 124 \\
\hline Hip & 0.48 & 3 & -25 & 25 \\
\hline Patella & 0 & 133 & 10.5 & 133 \\
\hline Condyles* &
\end{tabular}

*The condyle load was applied per condyle in the 3D model.

\subsubsection{2. $162^{\circ}$ Extension: Verify Muscle Forces in Equilibrium}

The same model process and validation criteria were used for the $162^{\circ}$ leg extension FE model as the $105^{\circ}$ model. See section 4.2.3.3 for further details. Due to the changes in load magnitudes for the $162^{\circ}$ case, the lateral condyle constraint load limit was based upon its largest applied load of $197 \mathrm{~N}$. Therefore, the magnitude of the lateral condyle constraint magnitude needed to be less than $39 \mathrm{~N}$ in order to meet the criteria set in section 4.2.3.3.

Table 4-8: $162^{\circ}$ extension validation results: $P=$ Predicted Force, $A=A B A Q U S$ Force.

\begin{tabular}{|c|c|c|c|c|}
\hline Constraint Type & x-comp. (N) & y-comp. (N) & z-comp. (N) & Magnitude (N) \\
\hline Hip Joint (P) & n/a & -117 & 158 & 197 \\
\hline \multicolumn{5}{|c|}{} \\
\hline Hip Joint (A) & -4.6 & -123 & -16.3 & 124 \\
\hline Lateral Condyle (A) & 24.0 & 0 & 11.5 & 27 \\
\hline
\end{tabular}


The results for the $162^{\circ}$ case had the lateral condyle magnitude within the acceptance range but the hip force magnitude was more than 5\% off from the biomechanically predicted magnitude. The error was traced back to the biomechanical simulation which again predicted forces that were not in static equilibrium. Even though there was a large disparity between the ABAQUS FE hip forces and the predicted hip forces, the distribution of forces from the ABAQUS FE model was chosen for the final FE model simulation. Despite the fact that the biomechanical simulation yielded forces not in static equilibrium, the muscle forces were determined directly from the EMG data which is a valid process. Therefore, the incorrectly predicted forces were assumed to occur at each joint (i.e. condyle, patella, and hip forces). Since each joint area was determined not to be an area of interest for the final FE model, due to high stress concentrations and complex geometry, it was reasonable to use the ABAQUS joint loads, which were in static equilibrium. Also, the force directions for the $162^{\circ} \mathrm{ABAQUS}$ load case were similar to the $105^{\circ}$ which intuitively made sense. Therefore, for multiple reasons, the ABAQUS FE model loading will be applied to the final FE model of the single leg extension exercise. Use of this modeling process in the future will require a fully-validated 3-D biomechanical model.

\subsubsection{LOADING AND BC - SUMMARY}

The final FE model for a single leg extension simulation will incorporate four distinct load cases (or steps, in ABAQUS) - phase I walking (heel on), phase II walking (toe off), $105^{\circ}$ leg extension, $162^{\circ}$ leg extension. Each of the walking phase loading and BCs will be duplicated from previously validated research (Deuel, 2007). The $105^{\circ}$ and $162^{\circ}$ leg extension cases will 
utilize the joint BC (Speirs et al., 2006) as well as the forces determined from validated ABAQUS FE models.

\subsection{BONE REMODELING: ALGORITHM}

The bone remodeling algorithm (Hazelwood et al., 2001) chosen for the final FE model simulation had already been converted into a user defined material (UMAT) code for ABAQUS (Hazelwood et al., 2007). However, the bone UMAT still required an additional modification before it could be used in conjunction with the final FE femur model. The change involved preconditioning the UMAT by allowing it to accept the initial porosity distribution input from the FE femur material data (Negrete, 2008).

\subsubsection{PRE-CONDITION DATA APPLICATION}

A bone UMAT which would accept user defined input variables had already been created (Hryce, 2010). The UMAT was able to accept a text file containing the initial porosity data which would then be applied to the FE model in ABAQUS. Therefore, the previously determined apparent density profile for the FE femur (Negrete, 2008) needed to be converted into a compatible text file.

A Matlab code was created which converted the apparent density distribution for the FE femur into a porosity distribution. First, the code would read the element, section, and material definition data from the ABAQUS input file created from the original FE femur. Next, the apparent density for each element set was converted into a porosity value. The conversion was based upon the maximum value of apparent density $\left(1.40 \mathrm{~g} / \mathrm{cm}^{3}\right)$ from the original FE model, and assuming a simple linear relationship between apparent density and porosity (a porosity of 0

corresponds to density $=1.4 \mathrm{~g} / \mathrm{cm}^{3}$, a porosity of 1.0 corresponds to a density=0.0 g/ $\mathrm{cm}^{3}$ ). Finally, 
the code would format the elemental porosity distribution into a text file which would be read directly into the UMAT. The fully annotated code can be seen in Appendix B.

\subsubsection{VALIDATION}

The final step in the FE leg extension simulation was validating that the bone UMAT was properly calibrated for the simulation. In order to validate the UMAT, an FE model with only the applied walking loads was run until steady state had been achieved. This was previously how the original UMAT code was validated (Hazelwood et al., 2007) in a study of marathon training regimens. The loading frequency for the validated UMAT was 3,000 cycles per day (cpd) which was maintained for the current study. The steady state value for the walking loads created a known initial condition for the simulation and simultaneously ensured that the model was in equilibrium for daily walking loads. This was important because it meant that any changes in the output of the model would be directly associated with the additional applied loads (e.g. the single leg extension exercise loads). Recall that the model included the daily walking loads in order to capture the reality that a subject undergoing the single leg extension exercise regimen would also continue normal amounts of walking. Since porosity was the driving variable in the bone UMAT, it was the one chosen to validate the model. Therefore, the bone UMAT for the final FE femur model would be validated if the steady state values due to walking loads only were within $5 \%$ of the previously determined initial porosity data.

A walking only FE model with four areas of interest (see figure 4-9) in the femoral neck of the femur was created - anterior, posterior, lateral, and medial. 

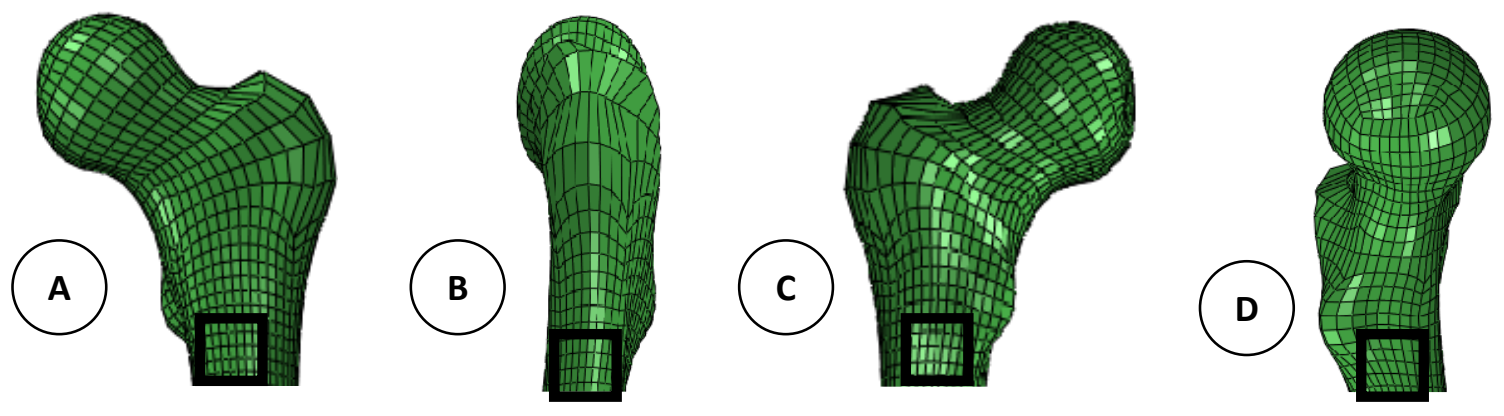

Figure 4-9: The femoral neck (A) anterior, (B) medial, (C) posterior, and (D) lateral areas of interest for the FE walking model used for validation.

The model assumed that one load cycle included the heel strike phase of walking for $3,000 \mathrm{cpd}$ and the toe off phase of walking for $3,000 \mathrm{cpd}$. It was then run for 140 load sequences (or 140 days; 1 load sequence $=1$ simulated day) in order to determine the steady state values due to walking loads only. To try and see how the walking magnitude affects the porosity values at the various areas of the femoral neck two additional models were run; one was run at $75 \%$ of the initial walking loads while the other was run at $110 \%$ of the initial walking loads. The comparative data for the three FE simulations in the four femoral neck locations can be seen below in figures 4-10 through 4-13. 


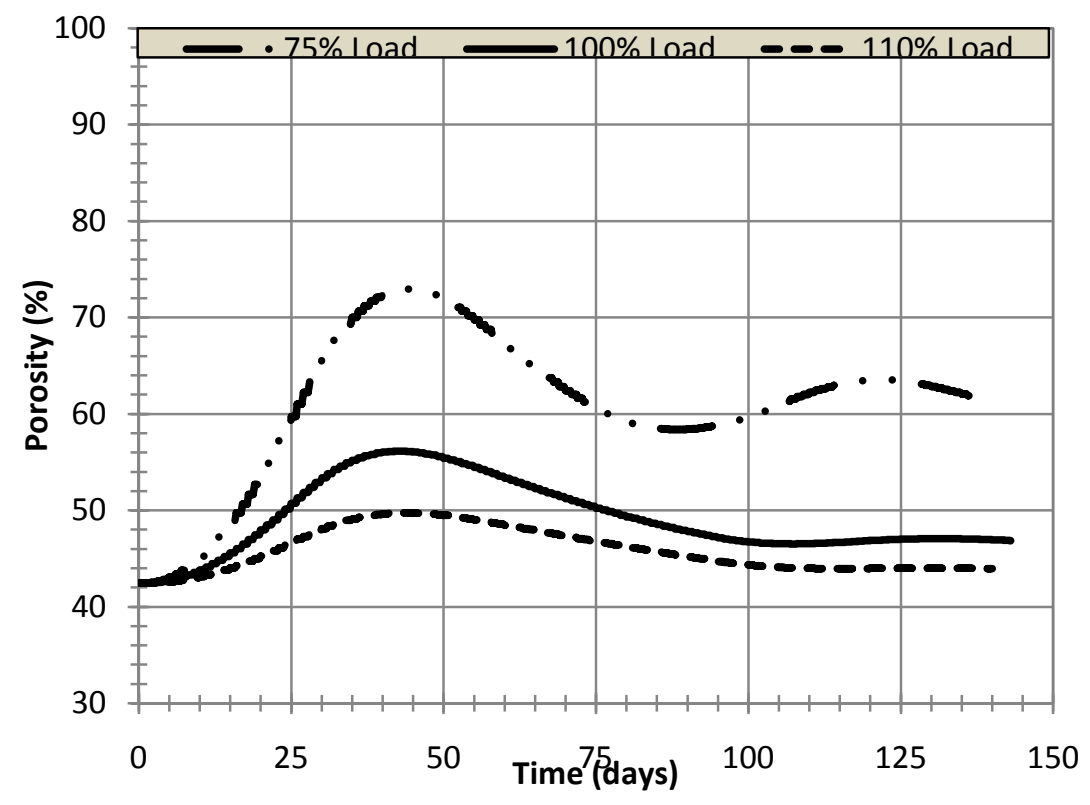

Figure 4-10: Comparison of steady state porosity values in the anterior femoral neck for a FE simulation with walking only loads.

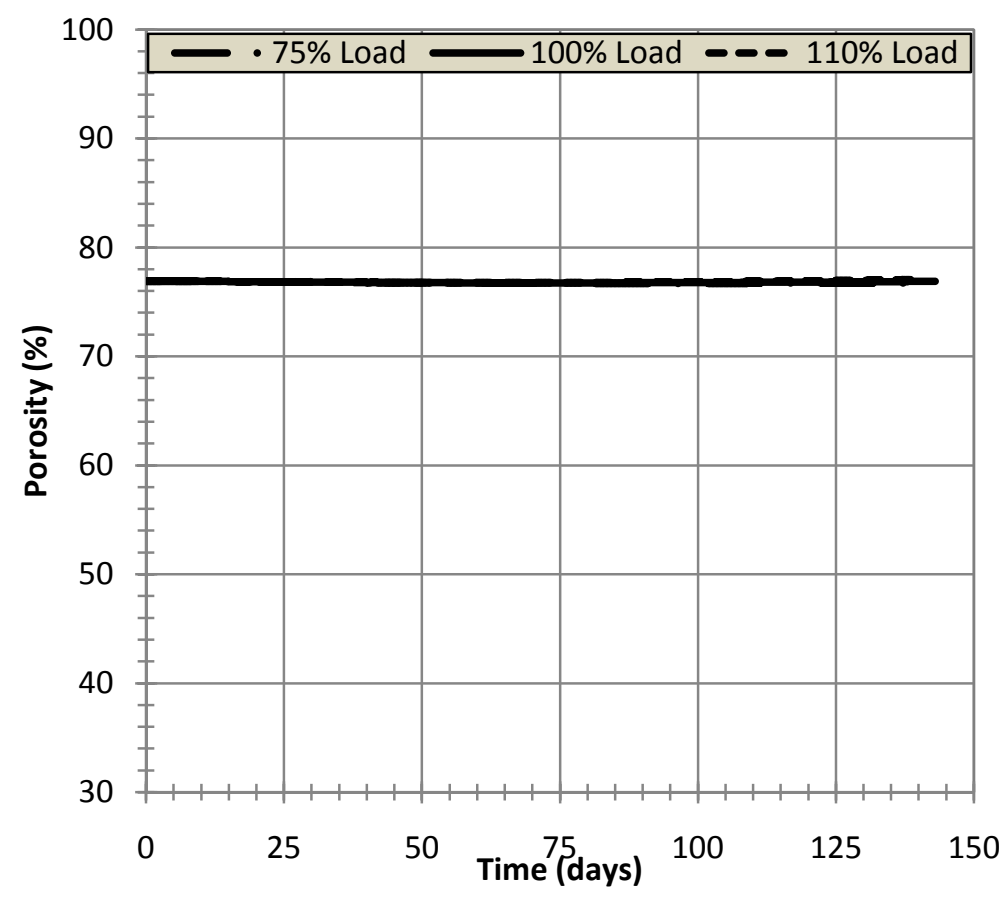

Figure 4-11: Comparison of steady state porosity values in the posterior femoral neck for a FE simulation with walking only loads. 


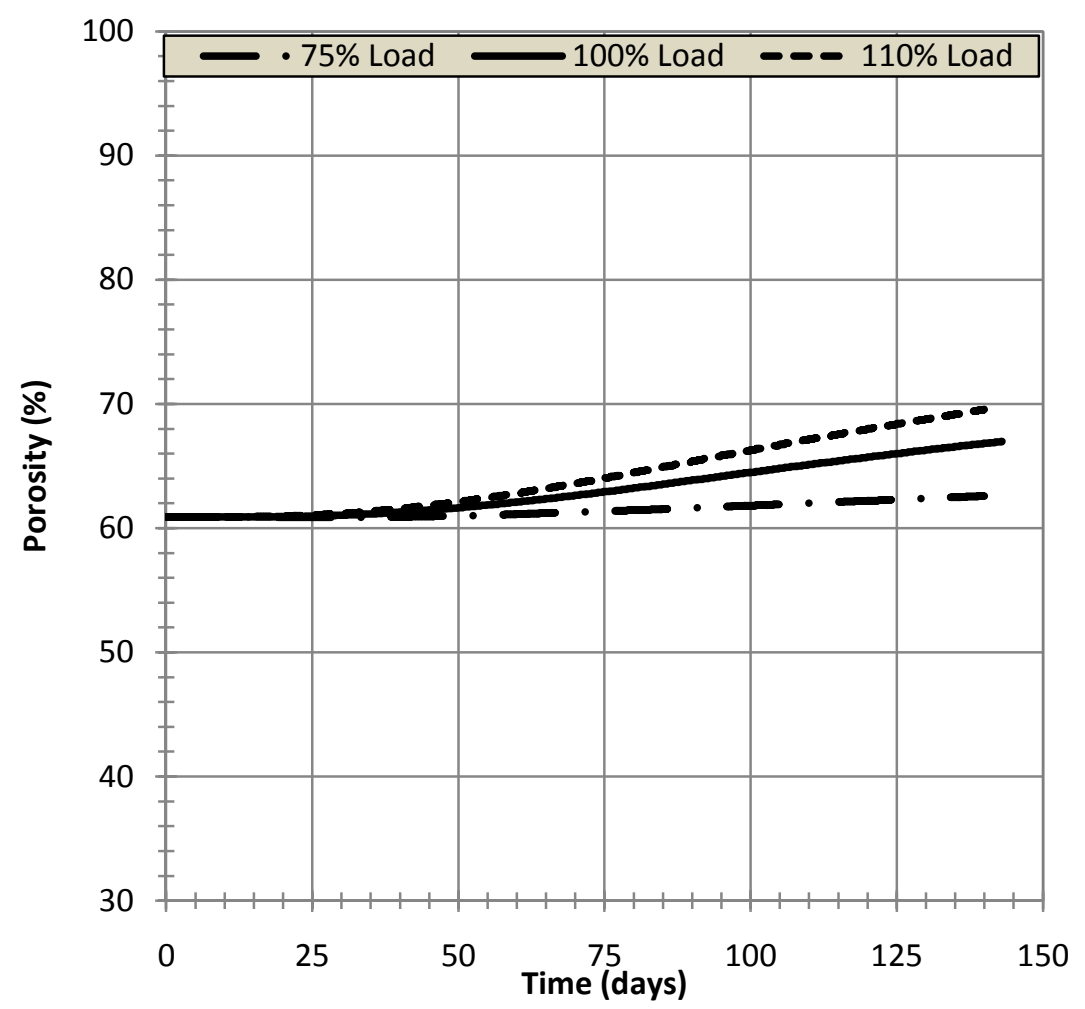

Figure 4-12: Comparison of steady state porosity values in the lateral femoral neck for a FE simulation with walking only loads.

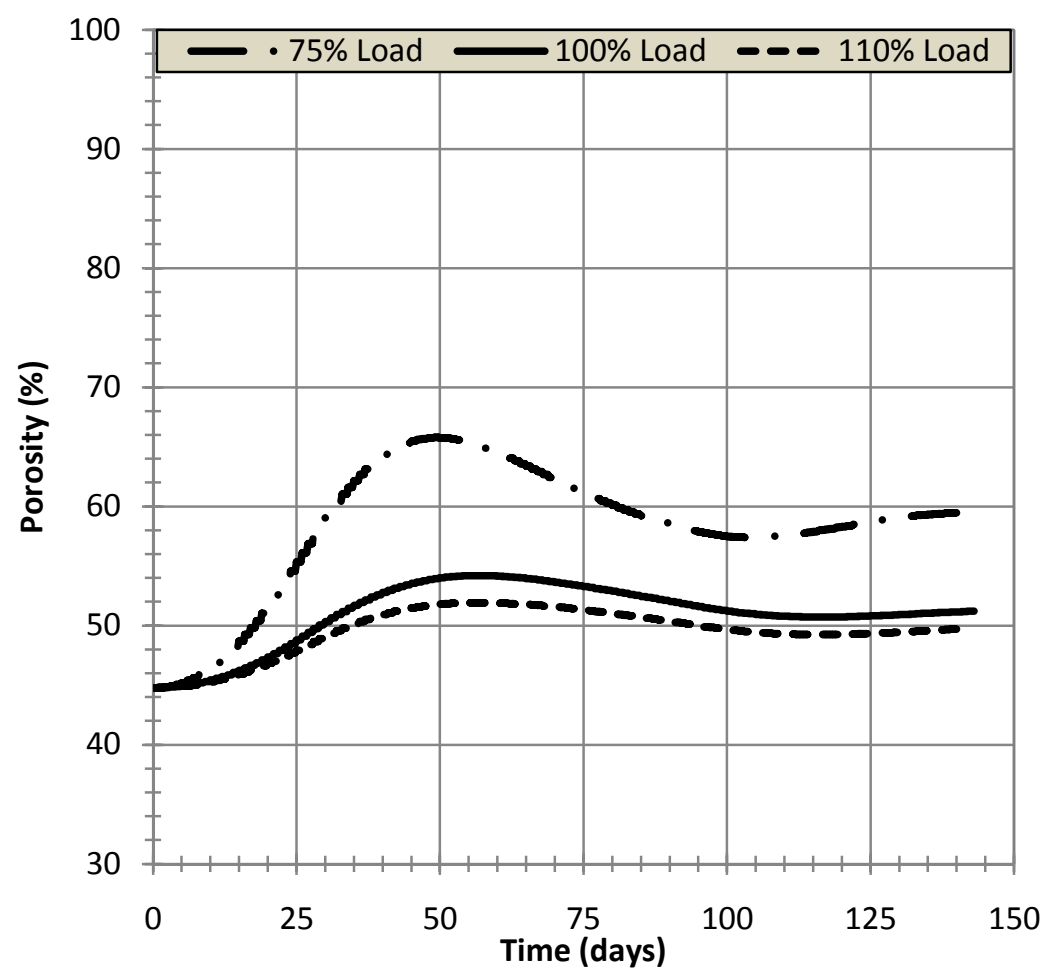

Figure 4-13: Comparison of steady state porosity values in the medial femoral neck for a FE simulation with walking only loads. 
The anterior and medial regions of the femoral neck trended in the same fashion - the increased load reduced the predicted porosity as well as the oscillatory effects seen in the lower loaded simulations. The posterior region demonstrated negligible change over the three load scenarios. As for the lateral region, it trended the opposite of the anterior and medial regions the increased load increased the predicted porosity as well as the oscillatory nature of the porosity profile from the lower loaded simulations. One explanation for the discrepancy in the medial region could be that in that region the bone may have been at equilibrium at the $75 \%$ load case. As the load was increased, the strains became too large and an increased response in remodeling was triggered in order to account for the increase in damage at that particular region. Another explanation could be that the different levels of porosity actually respond in different ways to the applied loads. The anterior and medial regions both have a porosity of approximately $45 \%$ and exhibit similar oscillatory behavior in response to the walking loads. The lateral region has a more porous average value, around $60 \%$, and exhibits not nearly as much oscillatory behavior as the previous regions and even trends in the opposite manner. Lastly, the posterior region has a very porous initial value of about $78 \%$ and exhibits no true response to the increase in load. These observations may be commented on later in the discussion section of chapter 6 if the final model also shows similar porosity trends.

Based upon the data collected, it was decided that the $110 \%$ loading scheme would be most representative for the final model walking loads. An additional FE simulation was run with the steady state time increased to 200 load sequences and the $110 \%$ walking loads applied. The initial and final porosity values were compared in each of the four regions and can be seen in Table 4-7. 
Table 4-9: Steady state porosity at $110 \%$ walking loads (simulated time $=200$ days).

\begin{tabular}{|c|c|c|c|}
\cline { 2 - 4 } \multicolumn{1}{c|}{} & $\mathbf{p}_{\text {INITIAL }}(\mathbf{\% )}$ & $\mathbf{p}_{\text {FINAL }}(\%)$ & \% Change \\
\hline Anterior & 42 & 43 & $\mathbf{1}$ \\
\hline Posterior & 77 & 78 & $\mathbf{1}$ \\
\hline Medial & 45 & 51 & $\mathbf{6}$ \\
\hline Lateral & 61 & 73 & $\mathbf{1 2}$ \\
\hline
\end{tabular}

Only the anterior and posterior areas of the femoral neck fall within the criteria for validation and therefore they will be the two areas used for the final FE simulation results.

\subsubsection{BONE REMODELING ALGORITH - SUMMARY}

The bone remodeling UMAT was successfully validated for the posterior and anterior parts of the femoral neck using the $110 \%$ of Hazelwood's previously validated walking loads (Hazelwood et al., 2007). The FE model porosity values reached their steady state values within 200 load sequences.

\subsection{FINAL FE MODEL}

The ABAQUS simulation for the final single leg extension FE model included four load cases: walking (phase 1), walking (phase 2), $105^{\circ}$ leg extension, $162^{\circ}$ leg extension. The portion of the ABAQUS input file containing the muscle loads for the model can be seen in Appendix C. The model was run for a total of 600 loading sequences (days). The first 200 days were run identical to the validated walking FE model above, with the heel strike and toe off walking loads applied at 3,000 cpd, in order to allow the areas of the femoral neck to equilibrate. After that, the $105^{\circ}$ and $162^{\circ}$ single leg extension exercise loads were added to the sequence at a rate of $30 \mathrm{cpd}$ for an additional 400 days ( 1yr). 


\section{CHAPTER 5: RESULTS}

\subsection{SINGLE LEG EXTENSION SIMULATION}

The results from the final single leg extension FE model are presented below. Three different loading frequencies of $30 \mathrm{cpd}, 3,000 \mathrm{cpd}$, and 300,000 cpd were input for the single leg extension simulation to see the impact it had on the final results. Recall equation 18 which defined the stimulus $(\varphi)$ for remodeling as a function of both strain (s) and load frequency $\left(R_{L}\right)$. The exponent q was set to a value of four for the final FE simulation.

$$
\Phi=\sum_{i=1}^{n} s^{q} R_{L i}
$$

Even with the single leg extension FE model being validated, verifying the model's response to changes in the load frequency was deemed a good sanity check for the final model. Strain could have also been used as a check but due to it being directly related to the applied loading, as well as the amount of loads which would have needed to be altered; it was easiest to vary the load frequency. The loading frequency needed to be increased two orders of magnitude in the two additional simulations because of the fourth order effect that the strain had on the remodeling stimulus. Both frequencies are very non-physical - the 3,000 cpd frequency would mean that the subject would do as many single leg extensions as walking in a single day. However, in order to elicit a bone remodeling response from the FE model, very high magnitudes of loading cycles needed to be chosen.

Although the medial and lateral sections of the femoral neck were not validated to the criteria discussed in chapter 4 , the results were still reported to show how the variation in cpd affects the model in the different areas of the femur. 


\subsection{POROSITY DATA}
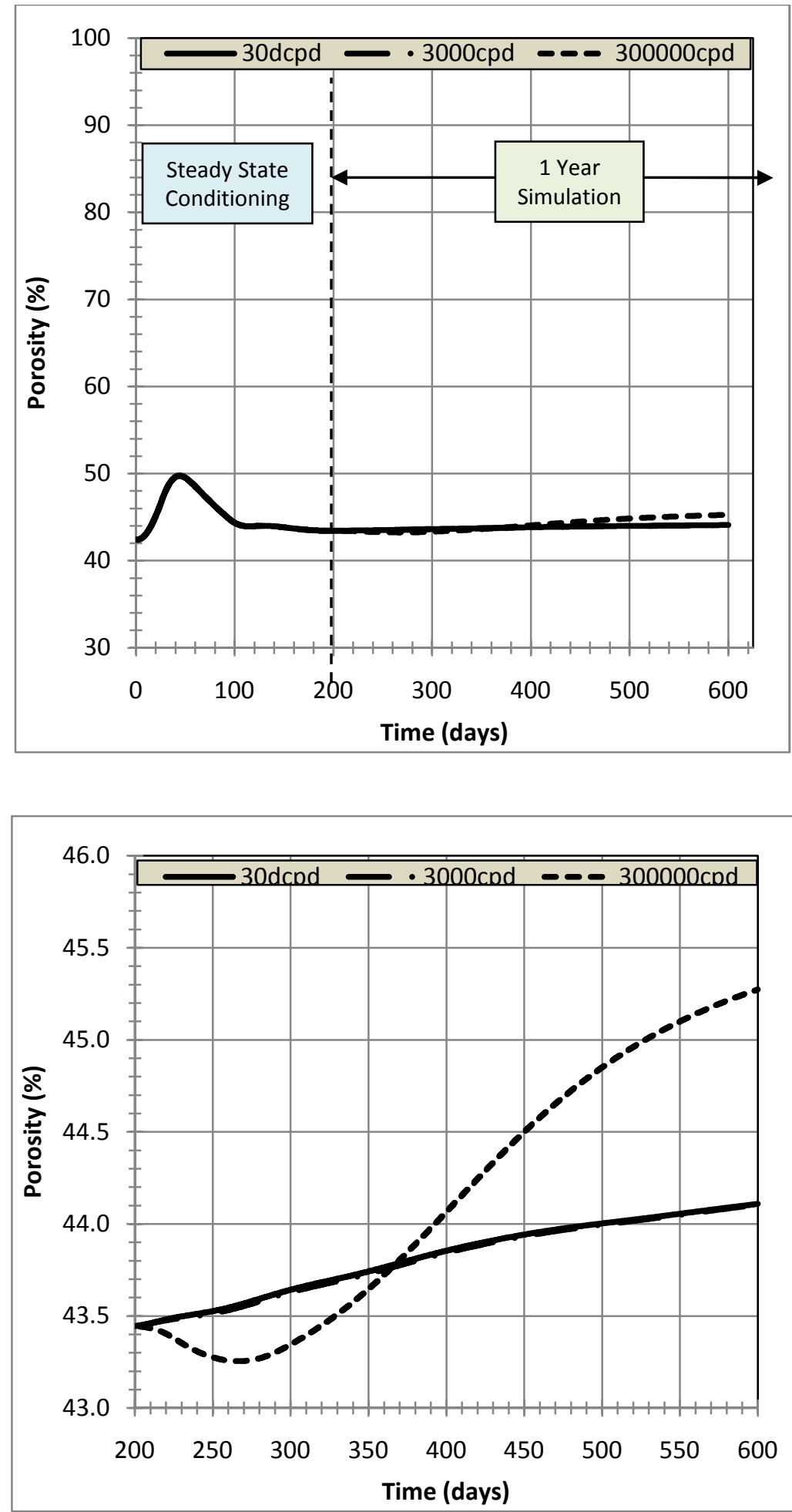

Figure 5-1: Anterior Femoral Neck Region: Porosity profile over (A) the 600 day simulation and (B) a year of daily single leg extension exercises at 30cpd, 3,000cpd, and 300,000cpd (scale zoomed in to show the changes in porosity). 

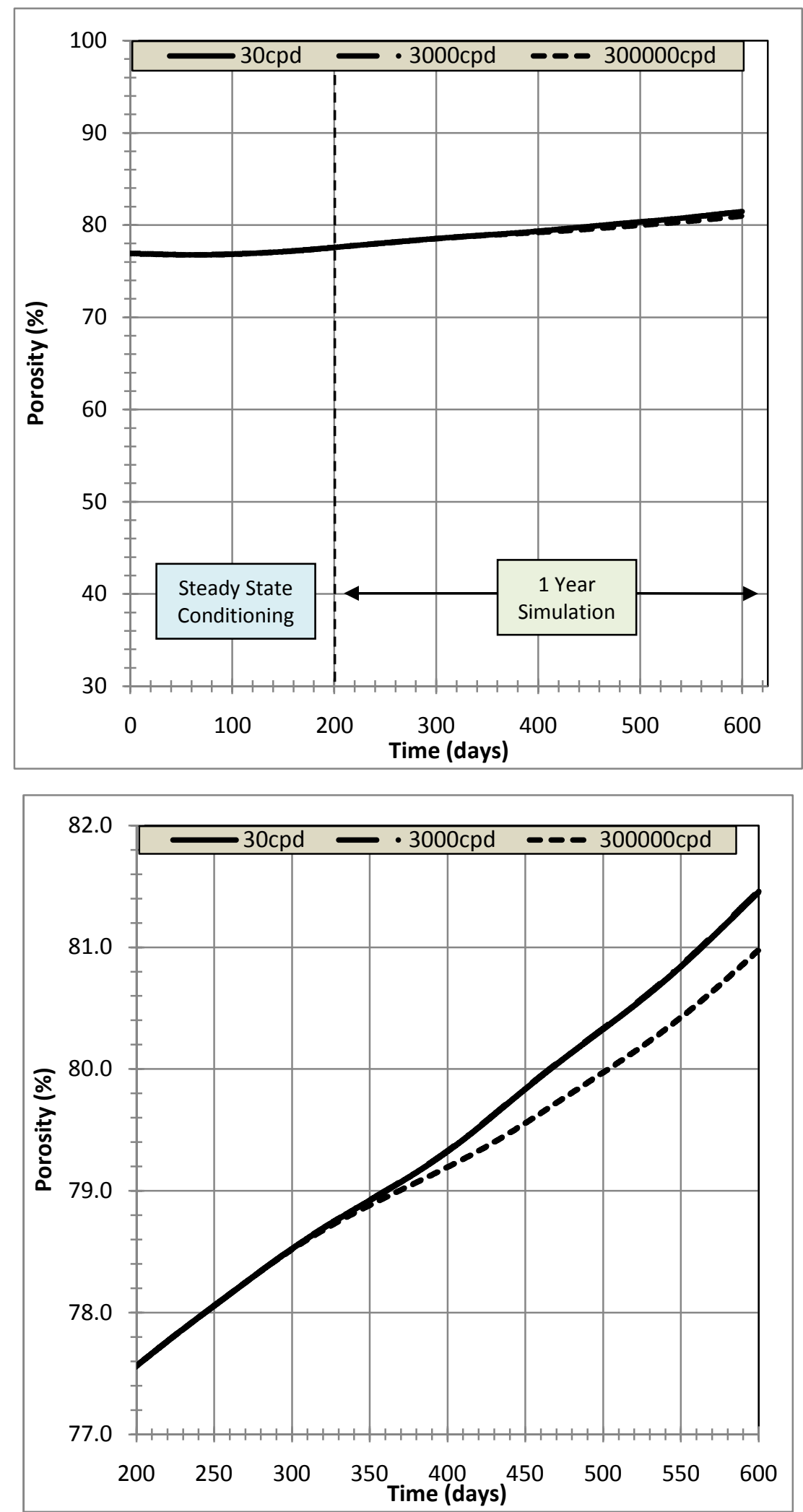

Figure 5-2: Posterior Femoral Neck Region: Porosity profile over (A) the 600 day simulation and (B) a year of daily single leg extension exercises at $30 \mathrm{cpd}, 3,000 \mathrm{cpd}$, and $300,000 \mathrm{cpd}$ (scale zoomed in to show the changes in porosity). 

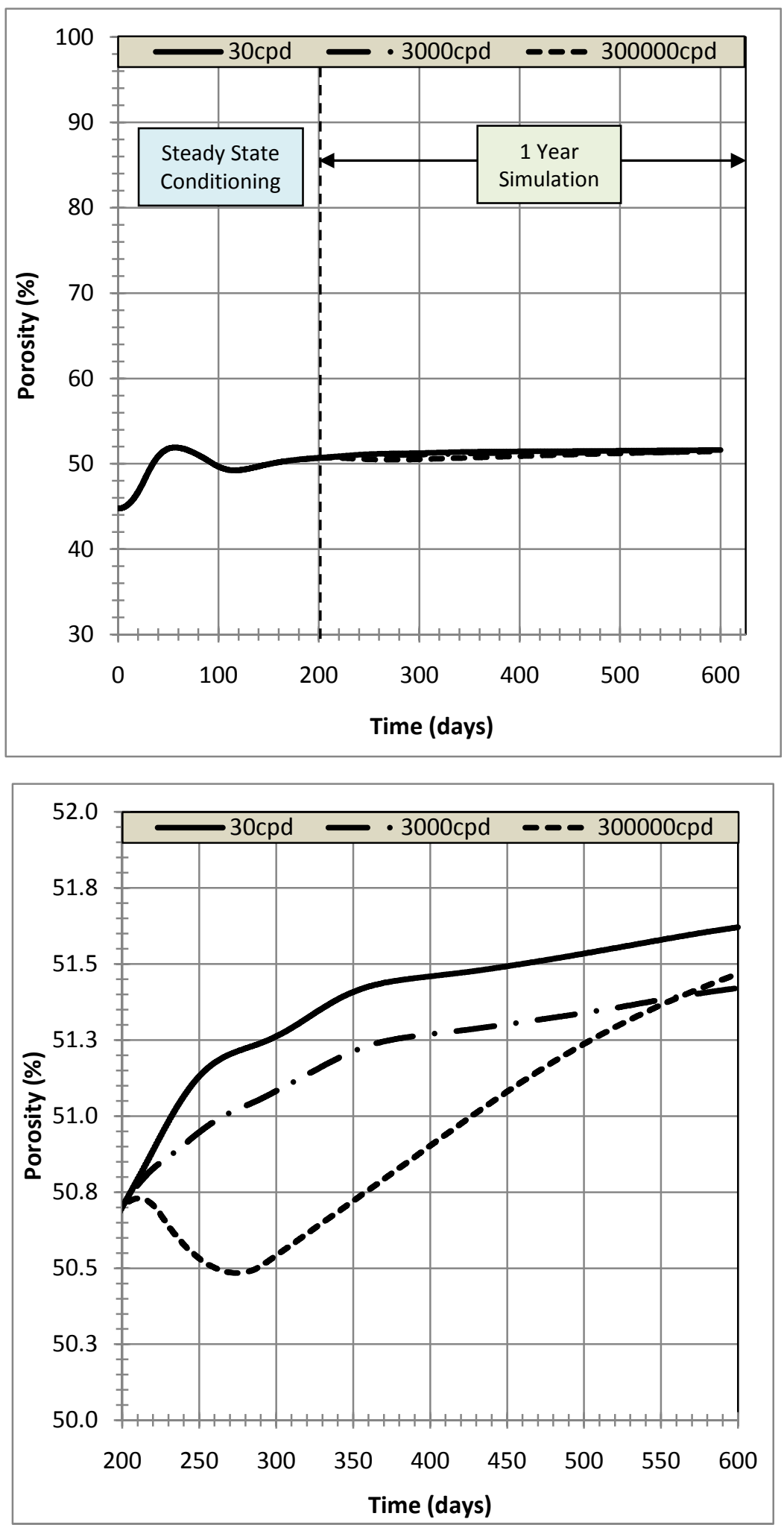

Figure 5-3: Medial Femoral Neck Region: Porosity profile over (A) the 600 day simulation and (B) a year of daily single leg extension exercises at 30cpd, 3,000cpd, and 300,000cpd (scale zoomed in to show the changes in porosity). 

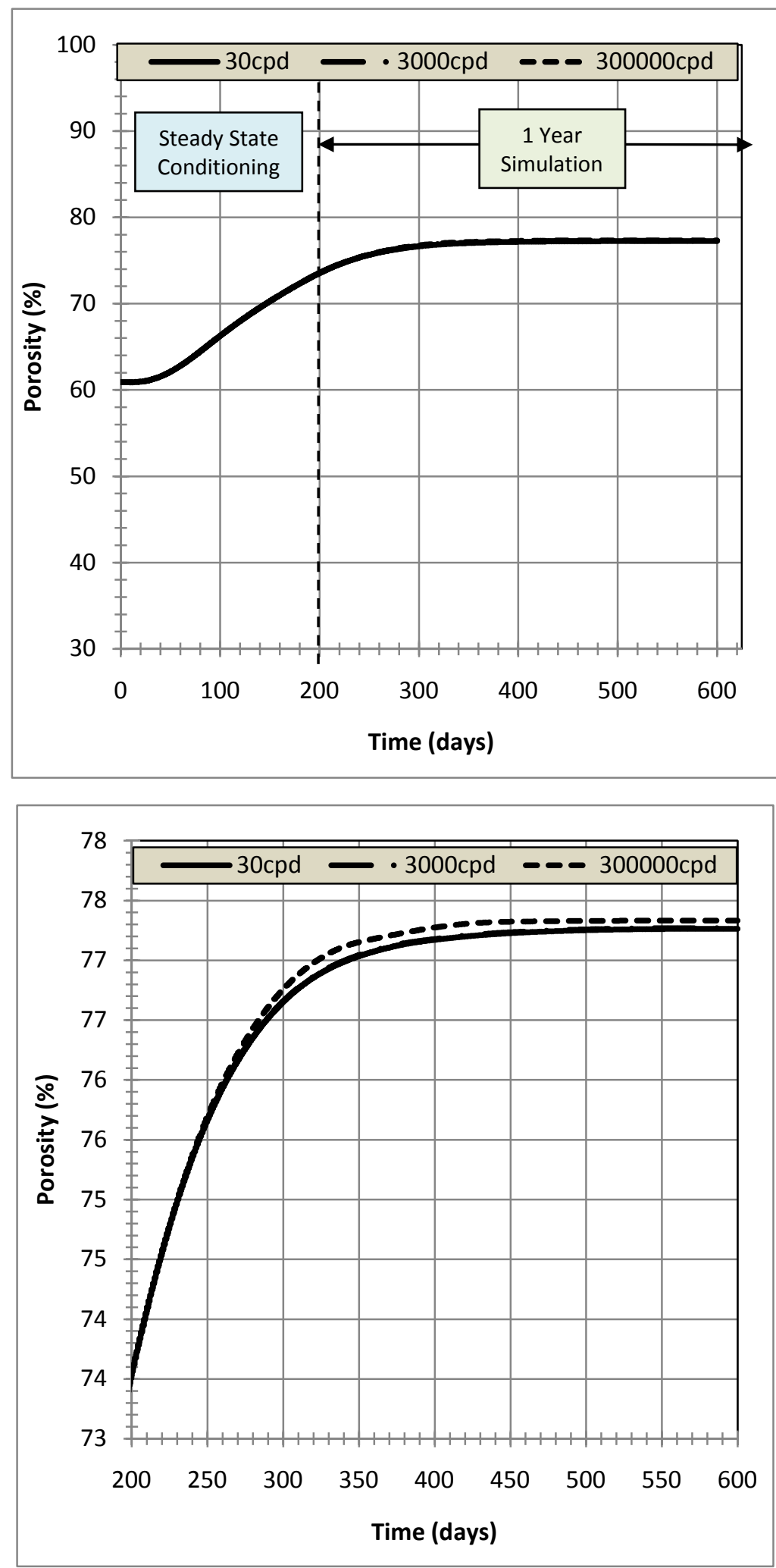

Figure 5-4: Lateral Femoral Neck Region: Porosity profile over (A) the 600 day simulation and (B) a year of daily single leg extension exercises at 30cpd, 3,000cpd, and 300,000cpd (scale zoomed in to show the changes in porosity). 


\subsection{NET CHANGE IN BONE POROSITY}

The net change in bone porosity over the single year simulation with a single leg extension exercise applied at $30 \mathrm{cpd}$ is summarized in table 5-1 for all four regions of the femoral neck. Recall that only the anterior and posterior areas were validated to have reached a set steady state value. The change in bone density for each region was only due to the addition of the single leg extension loads applied in addition to the daily walking performed in the one year FE simulation.

Table 5-1: Net change in bone porosity over a year of daily loading via single leg extension exercises applied at 30 cpd.

\begin{tabular}{|c|c|c|c|}
\hline Femoral Neck Region & $\mathbf{p}_{\text {INITIAL }}(\%)$ & $\mathbf{p}_{\text {FINAL }}(\%)$ & $\Delta$ \\
\hline Anterior & 43.45 & 44.11 & 0.66 \\
\hline Posterior & 77.57 & 81.46 & 3.89 \\
\hline Medial* $^{*}$ & 50.70 & 51.62 & 0.92 \\
\hline Lateral $^{*}$ & 62.84 & 66.84 & 4.00 \\
\hline
\end{tabular}

*Did not fully reach equilibrium after 200 days.

In all four regions, the addition of the single leg extension loading caused an increase in the porosity of the bone. This meant that loading the bone via exercise actually caused the BMD to decrease. The largest increase in porosity was seen in the lateral region with the smallest increase in the anterior region.

\subsection{VARIATION OF LOADING FREQUENCY}

The results due to the incremental increase of the loading frequency on the FE model simulation can be seen in figure 5-5. The figure shows the porosity values predicted after one year of single leg extension at the various load frequencies. 

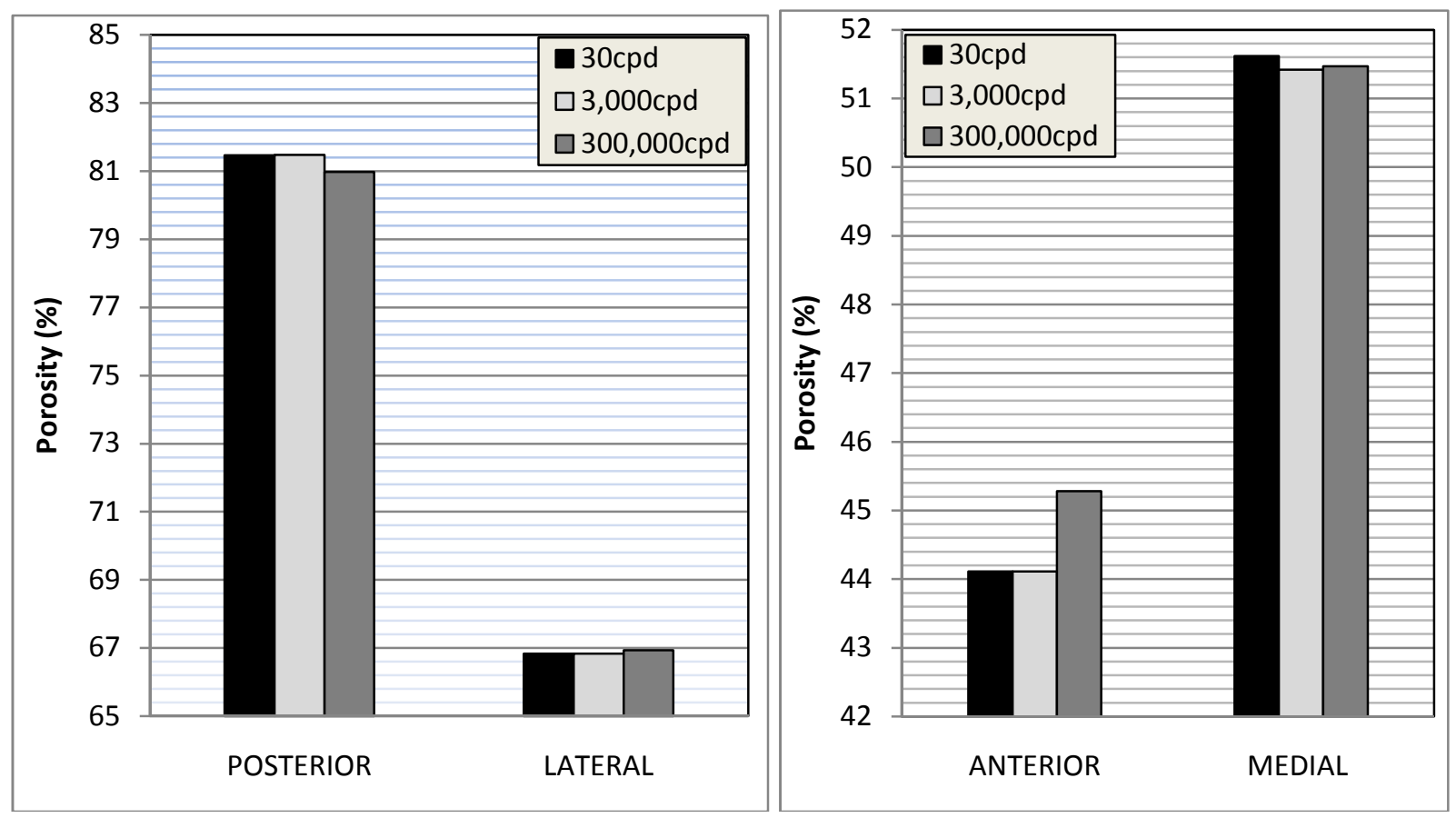

Figure 5-5: Comparison of femoral neck porosity after a one year simulation with varying frequencies: $30 \mathrm{cpd}, 3,000 \mathrm{cpd}$, and 300,000cpd.

There did not seem to be a consistent trend in the data at the femoral neck of the femur. The increase in load frequency caused an incremental net increase in porosity in the anterior and lateral regions and an incremental net decrease in the posterior and medial regions. There was not much observable change in the lateral region which had all three simulations within $1 \%$ of their final value after the first 150 loading cycles (days). The largest change was seen in the anterior region where the $300,000 \mathrm{cpd}$ porosity had an increase in porosity of approximately $1.5 \%$.

\subsection{RESULTS SUMMARY}

The single leg extension exercise which was run at the normal $30 \mathrm{cpd}$ loading frequency caused an increase in the bone porosity in all four regions of the femoral neck. The simulated results did not seem to follow Wolff's law with an increased load leading to a decrease in BMD. 
Increasing the loading frequency did affect the model results but not in a consistent fashion. The results presented above will be discussed further in the following section. 


\section{CHAPTER 6: DISCUSSION}

The results of the model presented have shown that it is possible to create a patient specific FE femur model capable of predicting mechanical changes in the bone based upon the following inputs: CT data, MRI data, EMG data, and a bone remodeling UMAT. The three final FE model results with varied single leg extension frequencies (30cpd, 3,000cpd, and 300,000cpd) are discussed in detail below and provide insight into the meaning of the results as well as the limitations of the model as a predictive tool.

\subsection{MECHANICAL STIMULUS}

The relationship between the mechanical stimulus and the stress / cycles per day of a given exercise is recalled from equation 18 , shown below.

$$
\Phi=\sum_{i=1}^{n} s^{q} R_{L i}
$$

For the single leg extension FE model, the only true variable in that equation was the loading frequency $\left(R_{L i}\right)$. That was because $\Phi$ was based upon previous research (Hazelwood et al., 2001) and the strain was directly determined from the loading conditions for the single leg extension which were also based upon previous research (Scott, 2008). For the current FE simulation, the exponent value was $\mathrm{q}=4$ which meant that the strain was much more influential in determining the mechanical stimulus the loading frequency. In other words, it would take a very large frequency to influence $\Phi$ which would consequently affect the end porosity profile of the bone. This relationship was reflected in the results of varying the frequency from $30 \mathrm{cpd}$ to $300,000 \mathrm{cpd}$ in all four regions of the femoral neck. From the results seen in figures 5-1 through 
$5-4$, the increase in cycle frequency did not always correlate to an end porosity which was less porous (i.e. more dense) after one year. Those results don't seem intuitively correct per Wolf's Law which simply states that bone adaptation is stimulated by mechanical loading of the bone. A closer look at the porosity results will be discussed before addressing the reasons for the apparent lack of agreement between the model and applied theory.

\subsection{POROSITY PROFILE}

The two areas of the femoral neck from which valid conclusions could be gleaned from were the anterior and posterior sections of the femoral neck. However, all four areas were compared and contrasted in order to see how the model responded to the applied loads.

All three FE models predicted changes in the bone's porosity in the femoral neck, yet there was no consistent trend among the four regions due to the increased loading frequency of the single leg extension. There were two orders of magnitude increments between the single leg extension load frequencies for the three models. Therefore, in comparison to the other higher frequency models, the $30 \mathrm{cpd}$ simulation was assumed to be representative of a condition where no single leg extensions were performed (i.e. load frequency $=0 \mathrm{cpd}$ ). That model served as the baseline condition with which the results of the 3,000 cpd and 300,000 cpd models were compared. In the anterior and lateral regions of the femoral neck, the 3,000 cpd model predicted a final porosity almost identical to the baseline whereas the $300,000 \mathrm{cpd}$ model predicted a net increase in the final bone porosity. As for the posterior and medial regions, the 3,000 cpd model predicted a final porosity almost identical to the baseline whereas the $300,000 \mathrm{cpd}$ model predicted a net decrease in the final bone porosity.

It would seem reasonable that per Wolff's Law, any loading in addition to the baseline walking loads of the FE femur would generate only two realistic possibilities: (1) no net effect or 
(2) a decrease in porosity. The reason an increase in porosity (i.e. density decrease) did not seem likely was due to equation 18 and its defining the mechanical stimulus ( $\left.\Phi^{\prime}\right)$ as the superposition of the product of the strain field and loading frequency due to the various loading conditions. Even if the single leg extension loads did not produce enough strain to stimulate bone growth, the walking loads applied would have continued to propagate the steady state values determined after the 200 daypre-conditioning of the femur. From that fact, one of two conclusions were made: (1) either the strain field produced by the single leg extension exercise created an overload situation which caused the bone to remodel the excessive amount of damage or (2) the 200 day steady state period was not sufficiently long enough to allow the FE femur to fully equilibrate to the applied walking loads. Due to the fact that the magnitude of forces applied to the femur during a single leg extension were lower as compared to daily walking, it was reasonable to assume the overload scenario described above was not valid and that what the model was predicting was actually a low frequency signal due to the walking loads not being at a pure steady state condition.

Although it was impossible to know whether the predicted values were physically relevant without actual patient data, the notion that the FE femur model was not allowed sufficient time to equilibrate was in line with previous results of a parametric study performed on the Hazelwood's bone remodeling algorithm (Hazelwood et al., 2001). In his study, Hazelwood compared steady state values of a femur which was allowed 400 loading cycles to equilibrate as well as a femur which was allowed 2000 days to equilibrate. The applied load in each case was constant at 445.8N. Figure 6-1 shows the results obtained from the comparison (recall 1 load sequence $=1$ simulated day). 


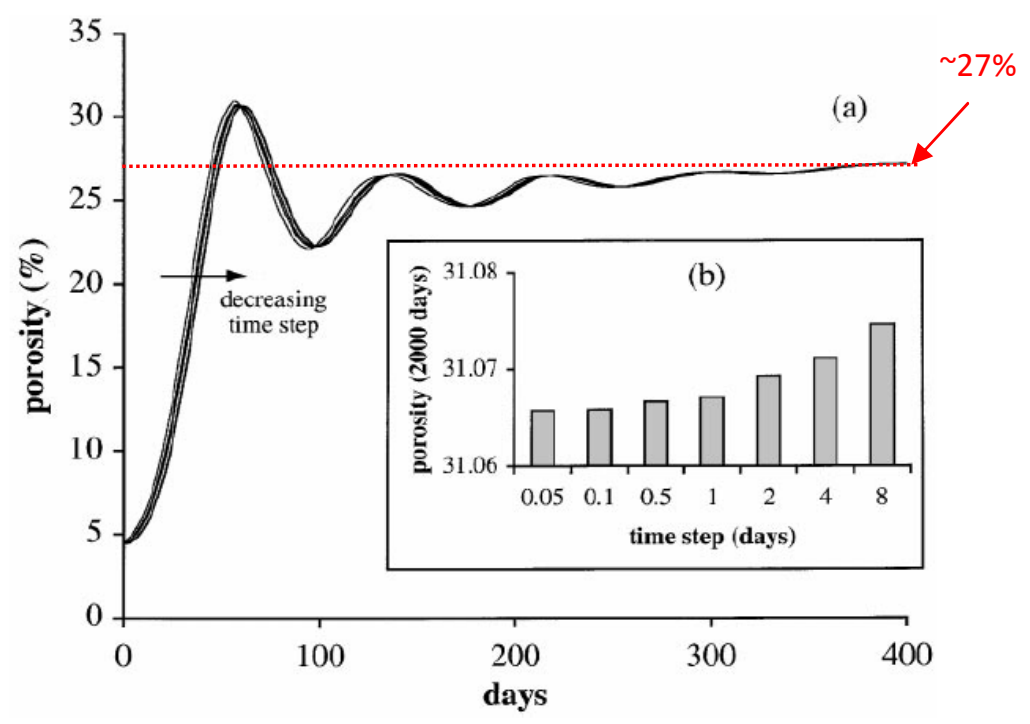

Figure 6-1: Porosity response (a) over the initial 400 days and (b) at 2000 days for a load of $445.8 \mathrm{~N}$ with varying time increments. (Hazelwood et al., 2001)

The figure showed that even though the steady state value seemed to only slightly vary from day 300 to day 400 that it was still not fully equilibrated at $27 \%$ and it would eventually settle at a value approximately $4 \%$ higher at $31 \%$ after 2,000 days. Although the FE femur in Hazelwood's simulation was created in the same manner as the FE femur for the current study, the results are still valid and provide valuable insight into the complexity of using bone remodeling algorithms in FE simulations of bone. Based upon the results seen in figure 6-1, it seems reasonable that the porosity profiles in the anterior, posterior, and medial femoral neck were more likely to have been caused by low frequency signals from the FE femur system trying to equilibrate than a physical increase/decrease in the femur's porosity. However, the lateral porosity profile which seems to have sufficiently equilibrated in the $300,000 \mathrm{cpd}$ model to a value higher than the baseline does not seem to follow that trend. Without actually running a longer simulation for both the $30 \mathrm{cpd}$ and $300,000 \mathrm{cpd}$ models, it would be very difficult to tell 
whether each model was in fact exhibiting low frequency responses or if equilibrium had truly been achieved.

\subsection{FE MODEL IMPLICATIONS}

Although the model was capable of capturing changes in the mechanical properties of the bone due to the applied loads, the inconsistent results above show that more work is needed to truly understand the limitations of its predictive capabilities. The lack of a consistent porosity trend could be a product of not allowing enough time for the single leg extension loading to fully equilibrate, as was seen in the steady state data discussed above. The model for each of the four regions in the femoral neck should be simulated to 2,000 days in order to gain a much more comprehensive picture of what is happening. The 2,000 day simulation could then be used to confirm or deny that a longer pre-conditioning period is needed in order to fully capture the steady state of the femur under normal walking loads. If simulation confirms that a longer preconditioning is necessary, then the model can be adjusted accordingly and re-run for a single year simulation with the additional single leg extension loads applied. The results would hopefully be more consistent and representative of the physical simulation but would still require an eventual physical experiment to be completely validated. However, if the 2,000 day data indicated that the current pre-conditioning of 200 load cycles was sufficient to establish a steady state condition, then the anomalies seen in the results would have to be explained from another source - maybe the model parameters need to be adjusted for a single leg extension exercise in either the remodeling code or the FE loading and BCs. In either situation, the single leg extension model presented would take another step forward. The knowledge gained in determining the proper steady state condition would lead to the next step in the evolution of the model. 
Another worthwhile improvement in the development of the FE model would be to simulate the effects of varying the applied strain in the model. Similar to the loading frequency variation, this could have non-physical applications (i.e. the applied loading is greater than the subject can output in reality). However, per equation 18, the mechanical stimulus is highly dependent upon the applied strain values for the single leg extension exercise given the $4^{\text {th }}$ order exponential relationship between the two. It would be another positive system check to try and verify how small changes in the applied strain affect the porosity results of the FE model.

Given the above discussion and observations, it does not seem realistic that the model would yield any relevant results in the current state. There are too many unknowns and the model has yet to be truly validated via experimentation. However, there are some viable options moving forward for the current FE model which would allow for some of the unknowns to be eliminated. Determining the true steady state condition of the FE femur under normal walking conditions as well as verifying the effect that stain has on the remodeling stimulus would greatly improve the validity of the model over its current state. 


\section{CHAPTER 7: FUTURE WORK}

The following section discusses various portions of the current study that could be improved upon in future implementations of the FE single leg extension simulation. The main focus will be on improvements to the leg extension predicted loads, the final FE leg extension model, and the bone remodeling simulation/UMAT.

\subsection{SINGLE LEG EXTENSION PREDICTED LOADS}

As discussed in section 4.2.3.3 and 4.2.4.2, the biomechanical loads that were predicted for the single leg extension were not in static equilibrium. In order to create a more accurate, as well as a more relevant model, the predicted forces need to be in static equilibrium prior to being imported into the ABAQUS FE model. Also, the predicted forces from the biomechanical simulation were for a 2D model which created some non-physiological loading in the final model. A fully validated 3D model of the muscles and loads involved in the single leg extension would be a great improvement in the future versions of the FE model.

The muscle forces provided from the biomechanical simulation represented approximately $90 \%$ of the active muscles for a single leg extension. Although a fully defined set of muscle forces would benefit the accuracy of the current FE model, the lack of integration of the ligaments and tendons would limit the overall potential of future simulations. Therefore, it would seem more important to see how the implementation of ligaments into the model would affect the model's output. Defining a set of ligament forces at each joint would help to reduce the constraint reaction forces as well as reduce the corresponding stress concentrations. This would help to create an FE model that is able to more fully describe the stress/strain field of the 
femur as opposed to the current model in which certain areas of the FE femur need to be disregarded due to non-physiologic loading.

Lastly, it would be ideal to actually determine the force generated at each individual muscle. Current technology has made it difficult to obtain minimally invasive EMG data for sub-surface

muscles, such as the vastus intermedius. Being able to determine individual muscle forces would go a long way in producing a more balanced loading of the femur and therefore a more accurate model for predicting the change in a bone's mechanical properties.

Additional future work improvements for the biomechanical simulation can also be seen in the thesis written my Matt Scott (Scott, 2008).

\subsection{SINGLE LEG EXTESION FE SIMULATION}

The single leg extension FE simulation future improvements can be broken down into three categories: the BCs, the muscle loads, and the final FE model. As for the actual FE femur model, the future work improvements can be seen in Estevan Negrete's thesis where the FE model of the femur was created (Negrete, 2008).

\subsubsection{BOUNDARY CONDITIONS}

The ideal condition for future versions of the FE model would be the anatomic BC described in section 2.2.3. This would require much more data in order to implement as well as a full set of fully balanced forces. The free BC would create more realistic results but it is possible that the results would not be significantly different from the current model; only a direct comparison between the two models can determine that. 


\subsubsection{MUSCLE LOADS}

The muscle loading is a very interesting and intricate problem to improve upon. It would be ideal to create a model which was able to fully model the attachment between the bone and muscle but further research would be needed in distributing the force over the attachment site. This would be a huge undertaking seeing as how some research has already shown that various muscles have "regions" of activation depending on the loading configuration (Soderberg et al., 1978). Although, with more and more data becoming available and technology allowing more and more intricate models to be simulated, this seems like a likely next step in the field of FE force application of muscles.

As for improving upon the muscle locations of the current FE model, including patient specific muscle attachment data would be extremely beneficial. Seeing as how the current FE model was developed for a specific bone, it seems like logical to try and obtain the physical locations of the muscle-to-bone attachments for the particular bone as well. It may be possible to collect this data via MRI or it may require an additional technologic method. Either way, future FE models of the single leg extension (and other potential lower extremity exercises) should try to incorporate this because using general muscle locations for a subject specific bone will eventually need to be eliminated. Also, the subject specific data could be used to develop the 3D force prediction model along with the EMG force data so it would have multiple uses for a more advanced FE simulation.

\subsubsection{FINAL MODEL}

There are two major areas of improvement that would not only greatly increase the validity of the FE model but also the potential implementation for research purposes. The first is a 
completely dynamic model that could fully model the entire range of motion for the single leg extension. The second involves trying to tie in the simulation of bisphosphonates and other pharmaceutical drugs, which are aimed and slowing the effects of osteoporosis, into the final simulation.

Integrating a dynamic single leg extension FE model would require a lot more development and research. It would require a very different approach to collecting and applying the muscle force data and BCs as well as the way the FE code is compiled. A truly dynamic model would also require much more initial data, time, and potentially money. A good intermediate step would be the addition of load steps to the isometric load cases. For example, adding load steps for every $15^{\circ}$ increment between $105^{\circ}$ and $162^{\circ}$. See figure $7-1$ for a comparison of the current model set up to the potential future model. This would be a more representative loading scenario for the femur and would translate into a more representative strain profile over the duration of the exercise.

As for the bisphosphonate implementation, the bone remodeling system parameters could be adjusted to include a dependence upon a new system variable that represents the particular drug. This would be similar to what Hazelwood has already attempted in a study which observed the effects of bisphosphonates in total hip replacement patients (Nyman et al., 2006). Another option would be to use documented data on how the drugs affect the bone and build those relations directly into the previous system parameters without an additional variable. This would offer the simulation another whole dimension in predictive capabilities but would also increase the complications in the eventual validation of the final FE model.

Another avenue that can be explored with the bone remodeling code in the future is the effects of modeling and how the external shape of the bones adapt and change. This phenomena 
was not captured in the presented body of work but could be used to model a completely different function of human bones.

Lastly, future models of the single leg extension exercise simulation could take a more in depth look at the damage trends in the femur. A full analysis of the damage trends caused by the single leg extension loads could lead to a hard conclusion about the increased predicted porosity - not enough time to equilibrate, too much load (i.e. bone is overloaded which leads to increased remodeling), or something not yet mentioned. Either way, the model validity would benefit from further investigation into the damage characteristics during a single leg extension exercise.

\subsection{BONE REMODELING SIMULATION/UMAT}

The best way to improve the bone remodeling code for future FE models is to validate the code for the single leg extension exercise. This would provide a physical set of data which would provide direct insight into the predicted histomorphometric parameters of the model. By directly comparing the model's parameters to the physical data, the individual parameters of the remodeling code could be validated. In particular, the current value of the exponent " $\mathrm{q}$ " in the mechanical stimulus equation (eq. 18) has been shown to yield good results for disuse when it's value is four, but has been cited by other authors as being as high as 15 . Being able to experimentally determine this value would drastically improve the predictive capability of the model. It would also eliminate the number of unknowns in the system, which could highlight the areas in which additional research and data would be required for further system validation.

Until a physical validation is able to be properly run for the single leg extension exercise, there are intermediate methods which could aid in improving the model. As more data for the various system parameters and their interactions with one another becomes available, the remodeling algorithm can be properly adjusted. Recall that the current model is based upon 
previous research as well as the system parameter study previously performed (Hazelwood et al., 2001). One example of this is the use of bisphophonates in the remodeling code which was discussed above. The current remodeling code by Hazelwood has the capability to incorporate this phenomenon in addition to the remodeling due to the loading simulation. In this way, the model allows for the incorporation of additional parameters (i.e. bisphophonates) as well as the fine tuning of the existing parameters (i.e. exponent q) in order to create a more appropriate FE model for predicting changes in the material composition of the femur due to a single leg extension exercise. 


\section{CHAPTER 8: CONCLUSION}

The results of the single leg extension FE simulation proved the concept and hypothesis that a subject specific FE model based upon CT, MRI, and EMG patient data can be used to predict changes in a BMD via porosity. This means that more in depth models are able to be created in the future. As discussed previously, proper definition of the loading and boundary conditions are paramount in obtaining any valid data from the FE simulation, especially with a remodeling algorithm that is based upon the stress/strain output of the model. Using the model creation method described above along with a good understanding of the physical experiment, a FE simulation can be created to predict the mechanical changes in the bone for a specific patient. The research presented in this paper is a good first step towards generating future models with predictive capabilities that will be able to aid in determining efficient ways of rehabilitating injured people, delaying the onset of osteoporosis in the elderly, and maintaining bone mass for astronauts to name a few.

However, understanding the limitations of the model and the interactions between loading and the mechanical changes in bone are essential if the simulation is to yield any valid data. From the results discussed previously, the two areas that will most affect a model's usefulness as a predictive tool are the steady state conditioning of the bone and the applied loading of the specific exercise. Allowing ample time for the FE model system to equilibrate to the daily walking loads will greatly reduce the low frequency signal input that can affect the final simulation output; in other words, it greatly increases the likelihood that any change in porosity is directly related to the single leg extension loading. The applied loading also can greatly affect the model output based upon the forth order relationship between the strain and the mechanical 
stimulus used for bone remodeling. Ensuring the proper load magnitudes and muscle locations on the femur will greatly increase the validity of the simulation results.

Even with the knowledge gained from the FE simulation and results, the only real way to know for sure how valid the results are is through physical validation of the simulated exercise and loading regiment. Due to the bone remodeling algorithm chosen which utilized model inputs composed of histomorphometric parameters, a physical validation of the exercise can actually be directly compared via the parameters of the model. This makes the single leg extension FE simulation able to adapt and be adjusted per physical results which could make it a very useful and powerful predictive tool. The validation would not only aid in determining the proper parameters for the single leg extension model but for the bone remodeling algorithm as well. There are still a lot of unknowns within the final FE model, but with the current advances in technology as well as researchers uncovering the mysteries of bone remodeling, it is only a matter of time before completely validated FE models will be able to be used in practical applications for science, medicine, and much more. 


\section{REFERENCES}

ABAQUS/CAE version 6.7, April 2007. ABAQUS Analysis User's Manual. Dassault Systems. 2007.

Baiotto, S., Labat, B., Vico, L., Zidi, M., "Bone remodeling regulation under unloading conditions: Numerical investigations." Computers in Biology and Medicine 39: 46-52, 2009.

Beaupré, G.S., Orr, T.E., Carter, D.R., “An approach for time-dependent bone modeling and remodeling - Theoretical development." Journal of Orthopaedic Research 8: 651-661, 1990a.

Beaupré, G.S., Orr, T.E., Carter, D.R., “An approach for time-dependent bone modeling and remodeling - Application: A preliminary remodeling simulation." Journal of Orthopaedic Research 8: 662-670, 1990b.

Behrens, B., Nolte, I., Wefstaedt, P., Stukenborg-Colsman, C., Bouguecha, A., "Numerical investigations on the strain-adaptive bone remodeling in the periprosthetic femur: Influence of the boundary conditions." BioMedical Engineering OnLine 8, 2009.

Bentolila, V., Boyce, T.M., Fyhrie, D.P., Drumb, R., Skerry, T.M., Schaffler, M.B., "Intracortical remodeling in adult rat long bones after fatigue loading." Bone 23: 275-281, 1998.

Bessho, M., Ohnishi, I., Matsuyama, J., Matsumoto, T., Imai, K., Nakamura, K., "Prediction of strength and strain of the proximal femur by a CT-based finite element method." Journal of Biomechanics 40: 1745-1753, 2007.

Bitsakos, C., Kerner, J., Fisher, I., Amis, A., "The effect of muscle loading on the simulation of bone remodeling in the proximal femur.” Journal of Biomechanics 38:133-139, 2005.

Brand, R.A., Crowninshield, R.D., Wittstock, C.E., Pedersen, D.R., Clark, C.R., van Krieken, F.M., "A model of lower extremity muscular anatomy." Journal of Biomechanics 140:304$310,1982$.

Brand, R.A., Pedersen, D.R., Davy, D.T., Kotzar, G.M., Heipie, K.G., Goldberg, V.M., "Comparison of hip force calculations and measurements in the same patient." The Journal of Arthroplasty 9:45-51, 1994.

Burr, D.B., Martin, R.B., Schaffler, M.B., Radin, E.L., "Bone remodeling in response to in vivo fatigue microdamage.” Journal of Biomechanics 18:189-200, 1985. 
Chen, G., Pettet, G., Pearcy, M., McElwain, D.L.S., "Comparison of two numerical approaches for bone remodeling." Medical Engineering \& Physics 29: 134-139, 2007.

Cody, D.D., Gross, G.J., Hou, F.J., Spencer, H.J., Goldstein, S.A., Fyhrie, D.P., “Femoral strength is better predicted by finite element models than QCT and DXA." Journal of Biomechanics 32: 1013-1020, 1999.

Cristofolini, L., Viceconti, M., Toni, A., Giunti, A., "Influence of thigh muscles on the axial strains in a proximal femur during early stance in gait." Journal of Biomechanics 28:617-624, 1995.

Crupi, V., Guglielmino, E., La Rosa, G., Vander Sloten, J., Van Oosterwyck, H., “Numerical analysis of bone adaption around an oral implant due to overload stress." Journal of Engineering in Medicine 218: 407-415, 2004.

Deuel, C.R., "Development of an adaptive 3-D model of the human femur to simulate bone remodeling following hip resurfacing and total hip arthroplasties.” PhD Dissertation; UC Davis, 2007.

Doblaré, M., García, J.M., "Application of an anisotropic bone-remodelling model based on a damage-repair theory to the analysis of the proximal femur before and after a total hip replacement." Journal of Biomechanics 34: 1157-1170, 2001.

Duda, G.N., Brand, D., Freitag, S., Lierse, W., Schneider, E., "Variability of femoral muscle attachments." Journal of Biomechanics 29: 1185-1190, 1996.

Duda, G.N., Schneider, E., Chao, E., "Internal forces and moments in the femur during walking." Journal of Biomechanics 30: 933-941, 1997.

Duda, G.N., Heller, M., Albinger, J., Schulz, O., Schneider, E., Claes, L., "Influence of muscle forces on femoral strain distribution.” Journal of Biomechanics 31:841-846, 1998.

Fyhrie, D.P., Schaffler, M.B., “The adaptation of bone apparent density to applied load.” Journal of Biomechanics 28:135-146, 1995.

García, J.M., Martinez, M.A., Doblaré, M., “An anisotropic internal - external bone adaptation model based on a combination of CAO and continuum damage mechanics technologies." Computer Methods in Biomechanics and Biomedical Engineering 4: 355-377, 2001.

García-Aznar, J.M., Rueberg, T., Doblaré, M., “A bone remodelling model coupling microdamage growth and repair by 3D BMU-activity." Biomechanics and Modeling in Mechanobiology 4:147-167, 2005. 
Going, S.B., Laudermilk, M, “Osteoporosis and strength training.” American Journal of Lifestyle Medicine 3:310-319, 2009.

Haapasalo, H., Sievanen, H., Kannus, P., Heinonen, A., Oja, P., Vuori, I., “Dimensions and estimated mechanical charatcteristics of the humerus after long-term tennis loading." Journal of Bone and Mineral Research 11 (6): 864-872, 1996.

Hart, R.T., Davy, D.T., Heiple, K.G., "Mathematical modeling and numerical solutions for functionally dependent bone remodeling." Calcified Tissue International 36:S104-S109, 1984.

Hartard, M., Haber, P., Ilieva, D. Preisinger, E., Seidl, G., Huber, J., "Systematic strength training as a model of therapeutic intervention: A controlled trial in postmenopausal women with osteopenia." American Journal of Physical Medicine and Rehabilitation 75:21-28, 1996.

Hazelwood, S.J., Martin, R.B., Rashid, M.M., Rodrigo, J.J., “A mechanistic model for internal bone remodeling exhibits different dynamic responses in disuse and overload." Journal of Biomechanics 34: 299-308, 2001.

Hazelwood, S.J., Castillo, A.B., "Simulated effects of marathon training on bone density, remodeling, and microdamage accumulation of the femur." International Journal of Fatigue 29: 1057-1064, 2007.

Heller, M.O., Bergmann, G., Deuretzbacher, G., Dürselen, L., Pohl, M., Claes, L., Haas, N.P., Duda, G.N., "Musculo-skeletal loading conditions at the hip during walking and stair climbing." Journal of Biomechanics 34:883-893, 2001.

Hernandez, C.J., Beaupré, G.S., Carter, D.R., “A model of mechanobiologic and metabolic influences on bone adaptation." Journal of Rehabilitation Research and Development 37:235244, 2000.

Hill, P.A., Orth, M., “Bone Remodelling.” British Journal of Orthodontics 25:101-107, 1998. Hoy, M.G., Zajac, F.E., Gordon, M.E., “A musculoskeletal model of the human lower extremity: The effect of muscle, tendon, and moment arm on the moment-angle relationship of musculotendon actuators at the hip, knee, and ankle." Journal of Biomechanics 23:157-169, 1990.

Hryce, T., "Modeling the long term effects of alendronate on bone mass preservation of the femur with articular surface and total hip replacement.” MS Thesis; Cal Poly, 2010. 
Huiskes, R., Weinans, H., Rietbergen, B., "The relationship between stress shielding and bone resorption around total hip stems and the effects of flexible materials." Clinical Orthopaedics and Related Research 274:124-134, 1992.

Jacobs, C.R., Levenston, M.E., Beaupré, G.S., Simo, J.C., Carter, D.R., "Numerical instabilities in bone remodeling simulations: The advantages of a node-based finite element approach." Journal of Biomechanics 28 (4): 449-459, 1995.

Kennedy, J.C., Hawkins, R.J., Willis, R.B., Danylchuck, K.D. “Tension studies of human knee ligaments. Yield point, ultimate failure, and disruption of the cruciate and tibial collateral ligaments.” Journal of Bone and Joint Surgery 58:350-355, 1976.

Keyak, J.H., Falkinstein, Y., "Comparison of in situ and in vitro CT scan-based finite element model predictions of proximal femoral fracture load." Medical Engineering \& Physics 25: 781-787, 2003.

Lanyon, L. "Osteocytes, strain detection, bone modeling, and remodeling." Calcified Tissue International 53: 102-107, 1992.

Li, J., Li, H., Shi, L., Fok, A.S.L., Ucer, C., Devlin, H., Horner, K., Silikas, N., “A mathematical model for simulating the bone remodeling process under mechanical stimulus." Dental Materials 23: 1073-1078, 2007.

Martin, R.B., "Porosity and specific surface of bone." Critical Reviews in Biomedical Engineering 10: 179-222, 1984.

Martin, R.B., Burr, D.B., Sharkey, N.A., Skeletal Tissue Mechanics. New York, NY; SpringerVerlag New York, 1998.

Materialise, MIMICS 11.0 Help Pages. 2007.

Matlab, February 2008. SimMechanics: For use with Simulink®. User's Guide. The Matworks Natick, MA. 2001-2004.

Menkes, A., Masel, S., Redmond, R.A., Koffler, K., Libanati, C.R., Gundberg, C.M., Zizic, T.M., Hagberg, J.M., Pratley, R.E., Hurley, B.F., "Strength training increases local bone mineral density and bone remodeling in middle-aged and older men.” Journal of Applied Physiology 5: 2478-2484, 1993.

Mullender, M.G., Huiskes, R., Weinans, H., “A physiological approach to the simulation of bone remodeling as a self-organizational control process.” Journal of Biomechanics 27:1389-1394, 1994. 
Negrete, E., "Development and implementation of subject-specific human femur finite element models." MS Thesis: Cal Poly, 2008.

Nyman, J.S., Rodrigo, J.J, Hazelwood, S.J., Yeh, O.C., Martin, R.B., “Predictions on preserving bone mass in knee arthroplasty bisphosphonates." The Journal of Arthroplasty 21:106-113, 2006.

Peng, L., Bai, J., Zeng, X., Zhou, Y., "Comparison of isotropic and orthotropic material property assignments on femoral finite element models under two loading conditions." Medical Engineering \& Physics 28: 227-233, 2006.

Phillips, A.T.M., "The femur as a musculo-skeletal construct: A free boundary condition modelling approach.” Medical Engineering \& Physics 31:673-680, 2009.

Phillips, A.T.M., "Numerical modelling of the pelvis and acetabular construct following hip arthroplasty.” PhD Dissertation; University of Edinburgh, 2005.

Phillips, A.T.M., Pankaj, P., Howie, C.R., Usmani, A.S., Simpson, A.H.R.W., "Finite element modelling of the pelvis: Inclusion of muscular and ligamentous boundary conditions." Medical Engineering \& Physics 29:739-748, 2007.

Polgar, K., Gill, H.S., Viceconti, M., Muray, D.W., O’Connor, J.J., “Strain distribution within the human femur due to physiological and simplified loading: finite element analysis using the muscle standardized femur." Proceedings of the Institution of Mechanical Engineers, Part H: Journal of Engineering in Medicine 217:173-189, 2003.

Schileo, E., Taddei, F., Malandrino, A., Cristofolini, L., Viceconti, M., "Subject-specific finite element models can accurately predict strain levels in long bones." Journal of Biomechanics 40: 2982-2989, 2007.

Scott, M., "Development of an EMG based force prediction model of the extensor muscles of the human knee and subsequent contact forces on the femur.” MS Thesis; Cal Poly, 2008.

Shahar, R., Banks-Sills, L., Eliasy, R., "Stress and strain distribution in the intact canine femur: finite element analysis." Medical Engineering \& Physics 25: 387-395, 2003.

Simões, J.A., Vaz, M.A., Blatcher, S., Taylor, M., "Influence of head constraint and muscle forces on the strain distribution within the intact femur." Medical Engineering and Physics 22: 453-459, 2000. 
Soderberg, G.L., Dostal, W.F., “An elecromyographic study of the three parts of the gluteus medius during activities of daily living." Journal of the American PhysicalTherapy Association 58:691-696, 1978.

Souminen, H., "Muscle training for bone strength.” Aging Clin Exp Res 18 (2): 85-93, 2006.

Speirs, A.D., Heller, M.O., Duda, G.N., Taylor, W.R., "Physiologically based boundary conditions in finite element modeling." Journal of Biomechanics 40: 2318-2323, 2006.

Stolk, J., Verdonschot, N., Huiskes, R., "Hip-joint and abductor -muscle forces adequately represent in vivo loading of a cemented total hip reconstruction." Journal of Biomechanics 34: 917-926, 2001.

Taddei, F., Cristofolini, L., Martelli, S., Gill, H.S., Viceconti, M., “Subject-specific finite element models of long bones: An in vitro evaluation of the overall accuracy." Journal of Biomechanics 39: 2457-2467, 2006a.

Taddei, F., Martelli, S., Reggiani, B., Cristofolini, L., Viceconti, M., "Finite-element modeling of bones from CT data: Sensitivity to geometry and material uncertainties." IEEE Transactions on Biomedical Engineering 53: 2194-2200, 2006 b.

Taylor, M.E, Tanner, K.E., Freeman, M.A.R., Yettram, A.L., "Stress and strain distribution within the intact femur: compression or bending?" Medical Engineering \& Physics 18: 122$131,1996$.

TrueGrid, April 2006. True Grid User's Manual. Compiled by Rainsberger R, XYZ Scientific Applications, Inc. 1992-2006.

Turner, A.W.L., Gilles, R.M., Sekel, R., Morris, P., Bruce, W., Walsh, W.R., "Computational bone remodeling simulations and comparisons with DEXA results." Journal of Orthopaedic Research 23:705-712, 2005.

Weinans, H., Huiskes, R., Grootenboer, H.J., “The behavior of adaptive bone remodeling simulation models." Journal of Biomechanics 25:1425-1441, 1992.

Wirtz, D.C., Pandorf, T., Portheine, F., Radermacher, K., Schiffers, N., Prescher, A., Weichert, D., Niethard, F.U., "Concept and development of an orthotropic FE model of the proximal femur." Journal of Biomechanics 36: 289-293, 2003.

Wolff, I., van Croonenborg, J.J., Kemper, H.C.G., Kostense, P.J., Twisk, J.W.R., “The effect of exercise training programs on bone mass: A meta-analysis of published controlled trials in pre- and postmenopausal women.” Osteoporosis International 9:1-12, 1999. 
Yosibash, Z., Trabelsi, N., Milgrom, C., "Reliable simulations of the human proximal femur by high-order finite element analysis validated by experimental observations." Journal of Biomechanics 40: 3688-3699, 2007.

Zernicke, R., MacKay, C., Lorincz, C., "Mechanisms of bone remodeling during weight-bearing exercise." Appl. Physiol. Nutr. Metab. 31: 655-660, 2006. 


\section{APPENDIX A}

\section{MUSCLE FORCE REDISTRIBUTION}

(Generating 3D muscle forces from 2D data) 


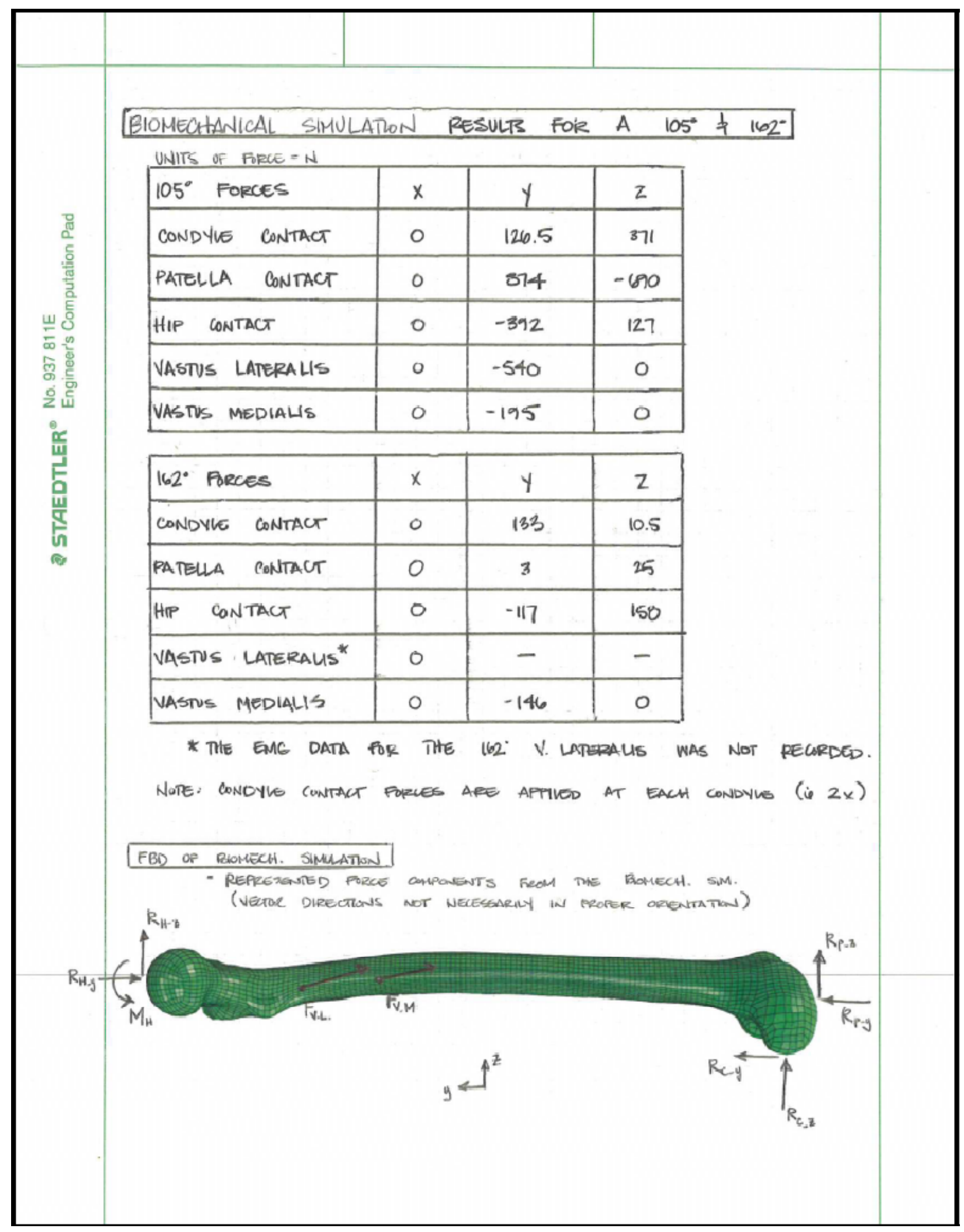




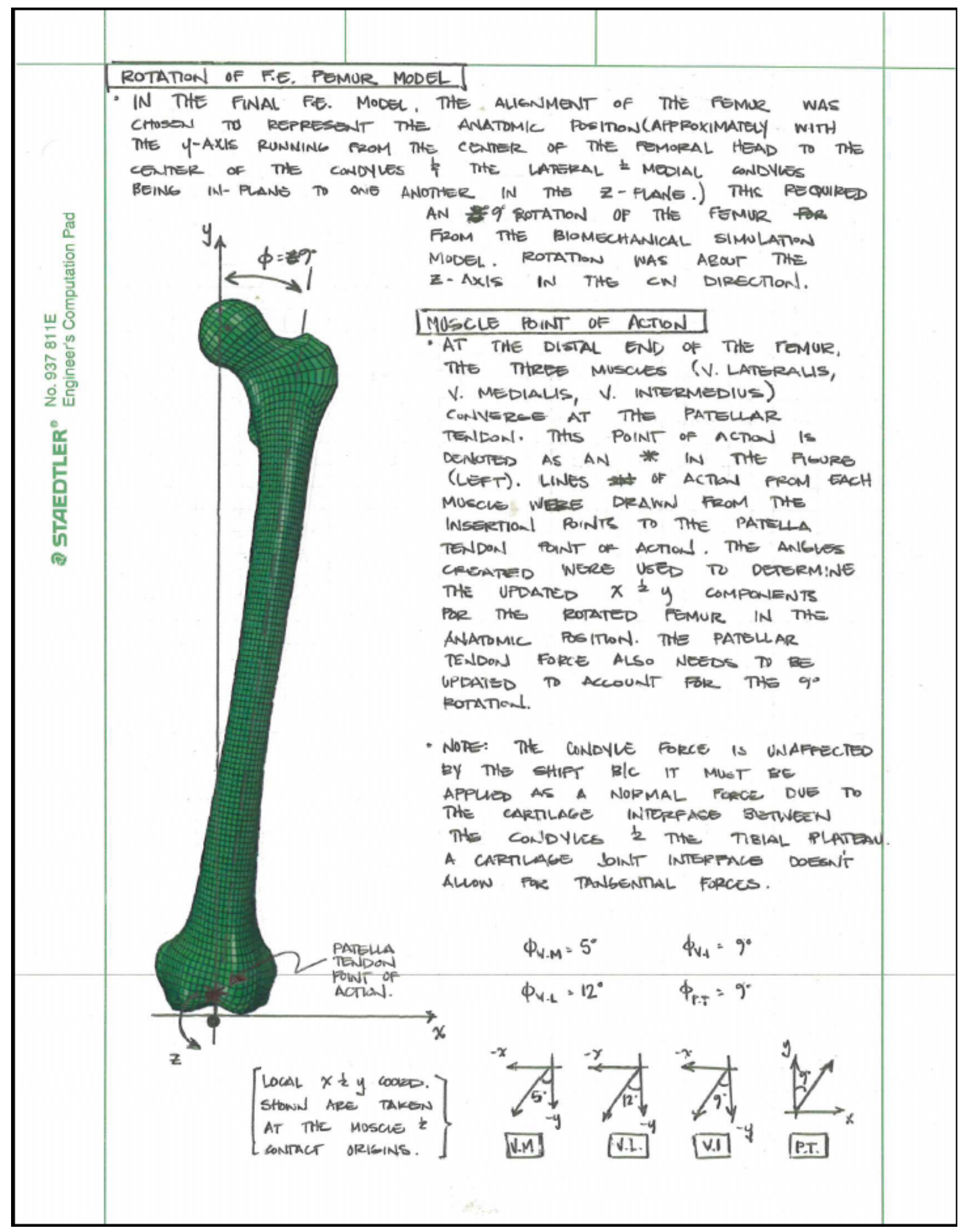









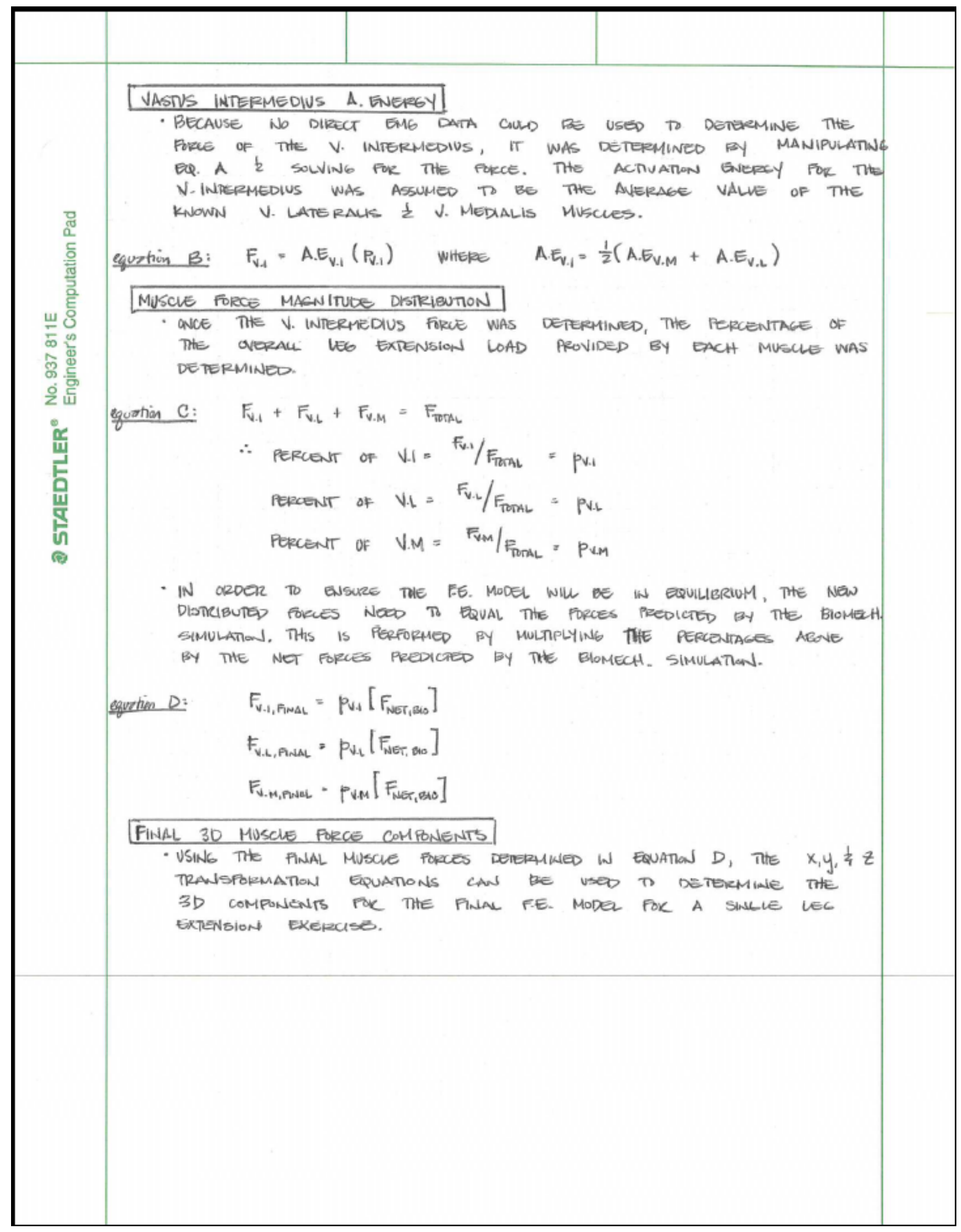




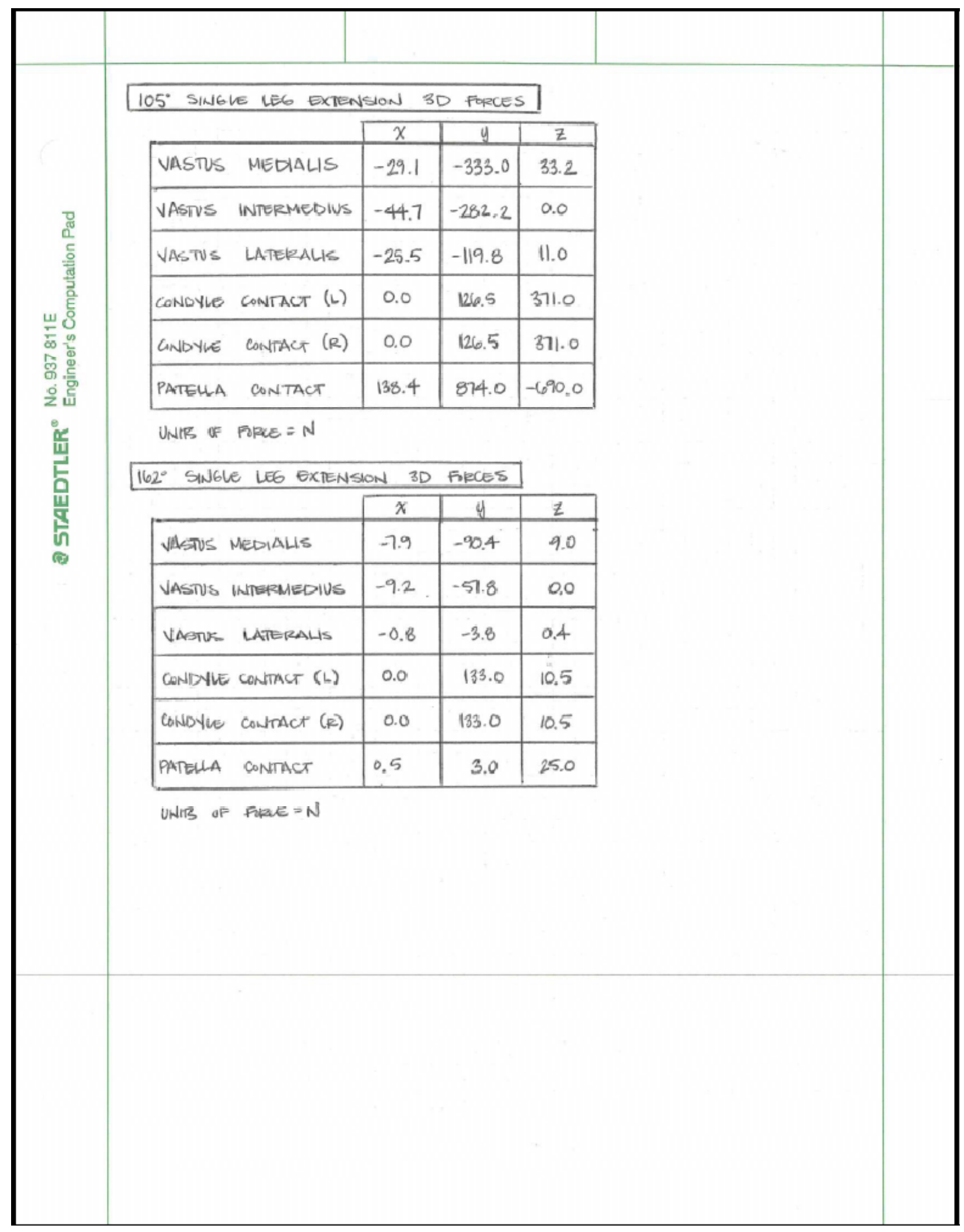




\section{APPENDIX B}

\section{MATLAB POROSITY PROFILE CODE}




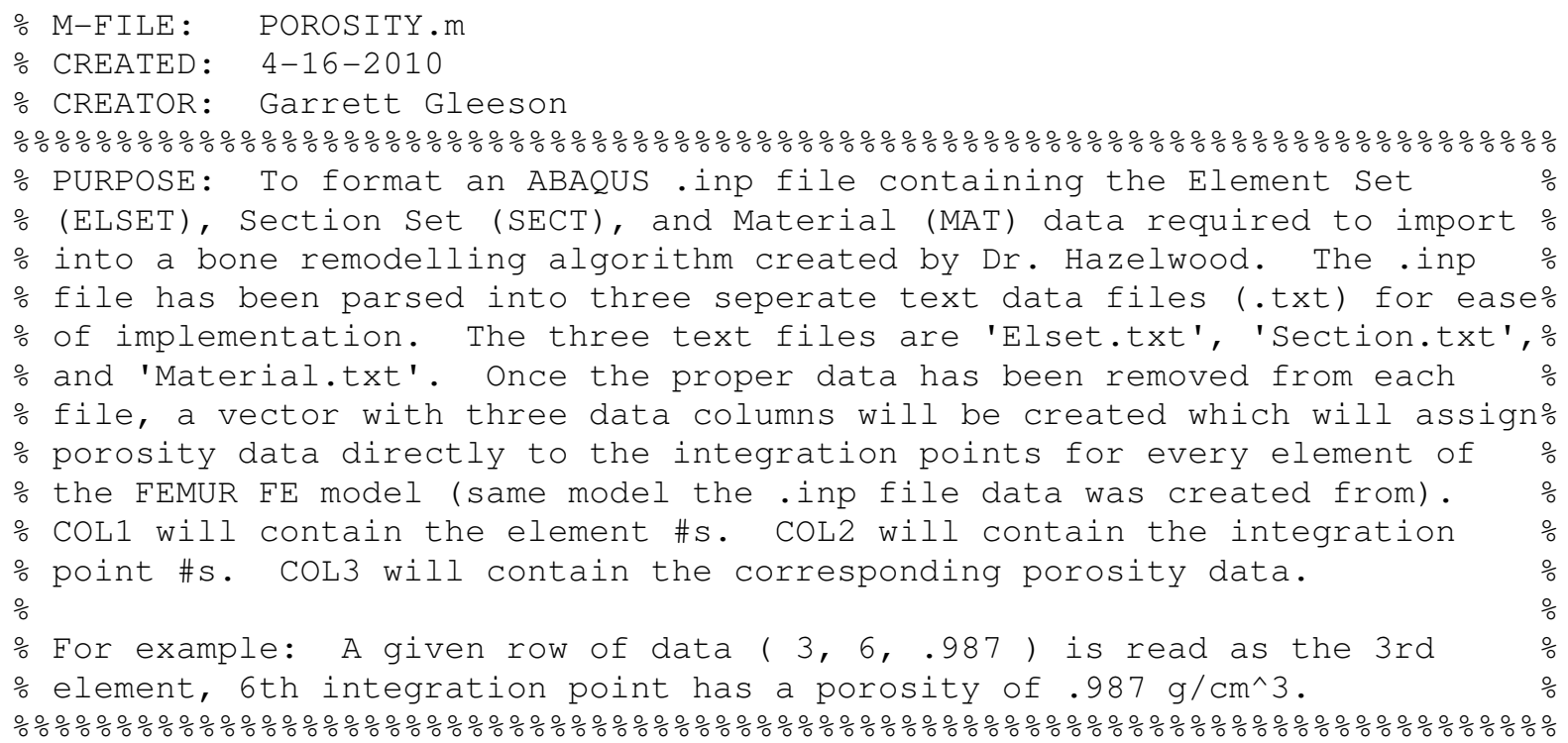

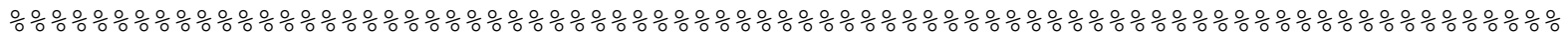

UNITS: (these are the units of the final vector, INT_POR)

$\div$

$\therefore$ Element \# $\quad($ COL1) 1$)$ NONE

$\div$ Integration Point \# (COL2) = NONE $\frac{\circ}{0}$

Porosity $\quad(\mathrm{COL} 3)=\mathrm{NONE} \%$

: Strain, Load1 (COL4) = strain $\%$

$\div$ Strain, Load2 (COL5) = strain $\%$

\% Strain, Load3 $(\operatorname{COL} 6)=\operatorname{strain} \div$

․ Strain, Load4 (COL 7$)=$ strain $\div$

\% Damage Potential (COL8) = cycles/day $\%$

$\div$ Equilibruim Damage $(\operatorname{COL} 9)=\mathrm{mm} / \mathrm{mm}^{\wedge} 2$

$\div$ \# Refilling BMUs $(\mathrm{COL} 10)=\mathrm{BMU} / \mathrm{mm}^{\wedge} 2$

# Resorbing BMUs $(\mathrm{COL} 11)=\mathrm{BMU} / \mathrm{mm}^{\wedge} 2$

Activation Freq. $\quad(\operatorname{COL} 12)=\mathrm{BMU} / \mathrm{mm}^{\wedge} 2 / \mathrm{day} \quad \%$

\% Act. Freq. History (COL13-COL106) = BMU $/ \mathrm{mm}^{\wedge} 2 / \mathrm{day} \%$

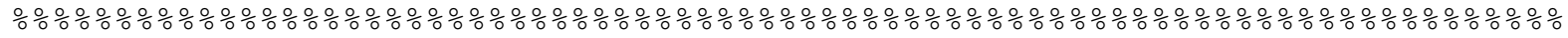

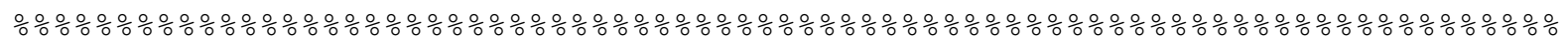

$\circ$

$\div$

$\frac{0}{0}$

ELEMENT SET VECTOR FORMATION

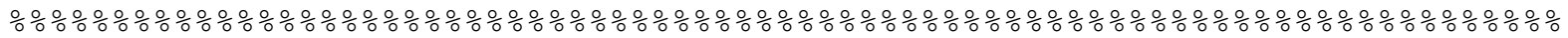

Import the element set data from the ABAQUS .inp text file. Note, the

o data will be formated as a cell.

raw_element_data = importdata('Elset.txt', '');

Initialize the vector containing the Element SET \# in COL1, the first

\% element \# in COL2, and the final element \# in COL3. There are a total

\% of 352 SETS per the ABAQUS . inp file.

ELSET=zeros $(352,3)$; 
\% Each set of raw elemental data contains two cells worth of data (Example o below) once it is read into the cell array 'raw_element_data'.

응

응

응

응

$\%$

\% The first data cell of each SET is useless and will not be used.

Therefore,

\% grab every even numbered line of code from the raw data as it is the line \% that contains the Element number limits which will define which Elements are

o contained in a given SET\#. (i.e. SET1 contains Elements 1-39)

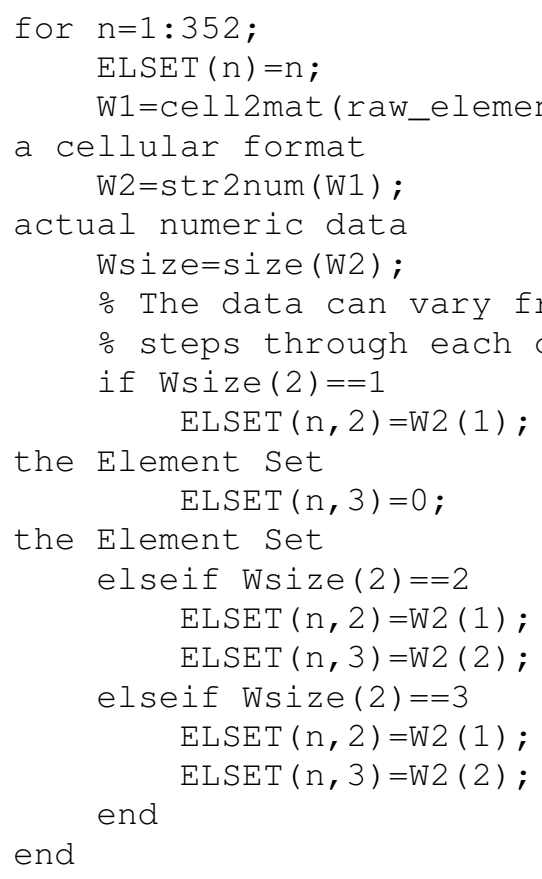




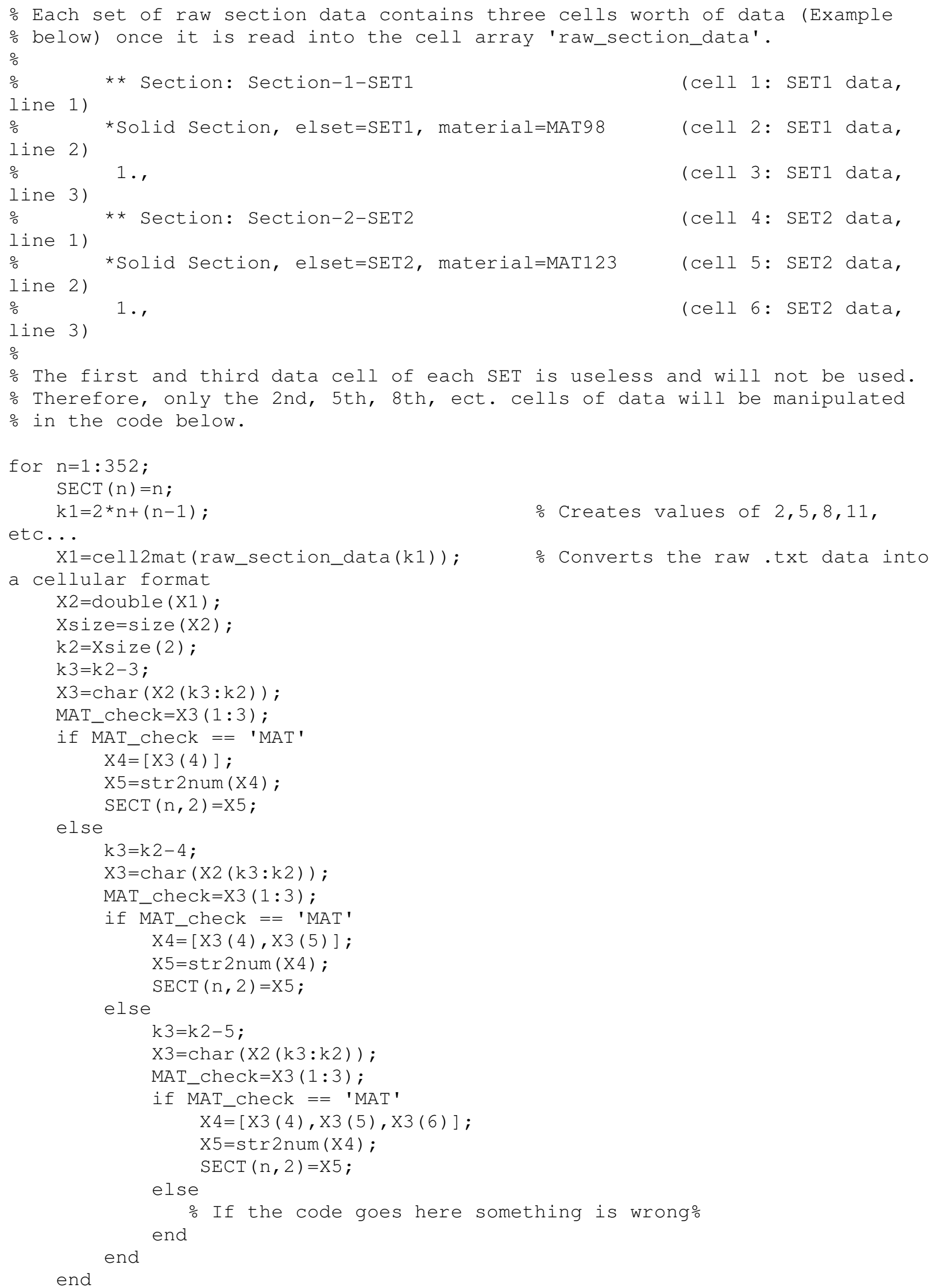




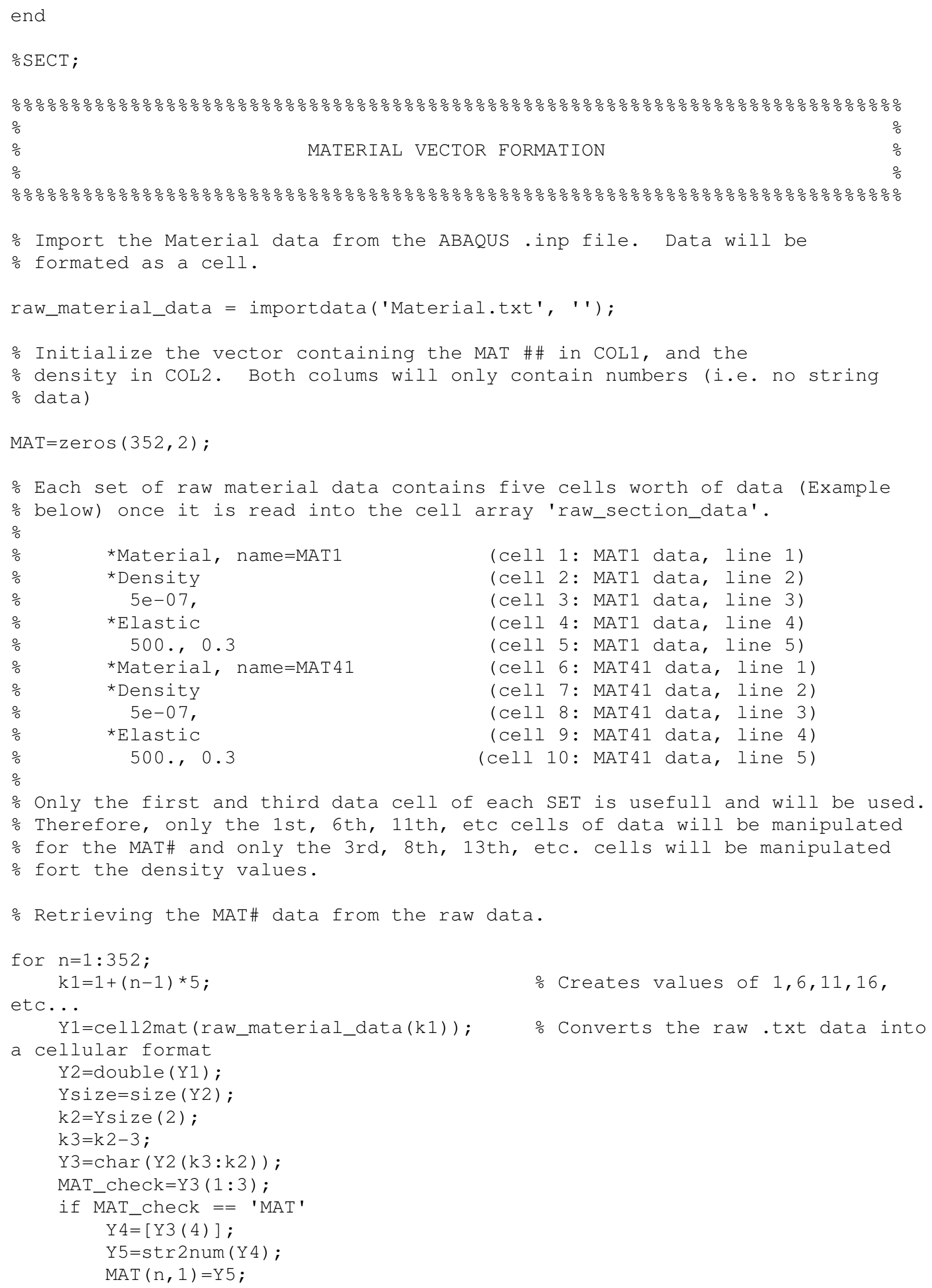




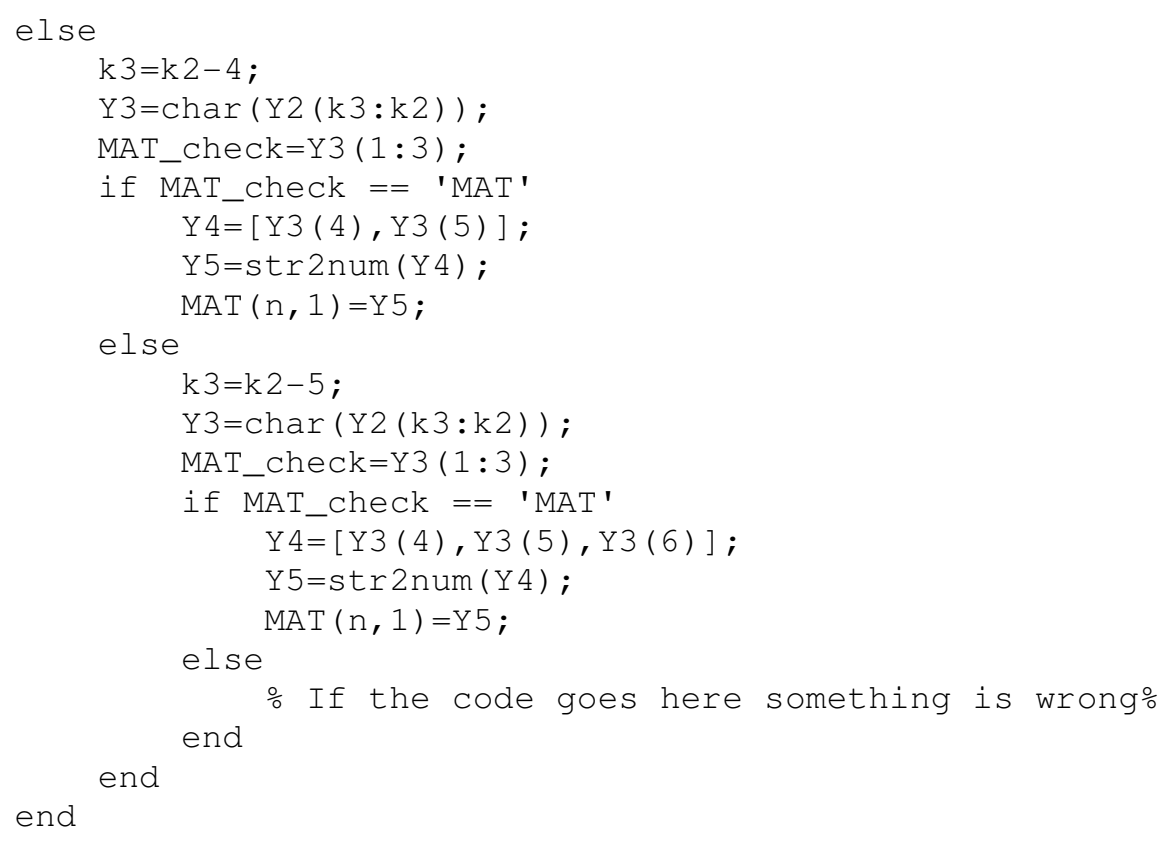




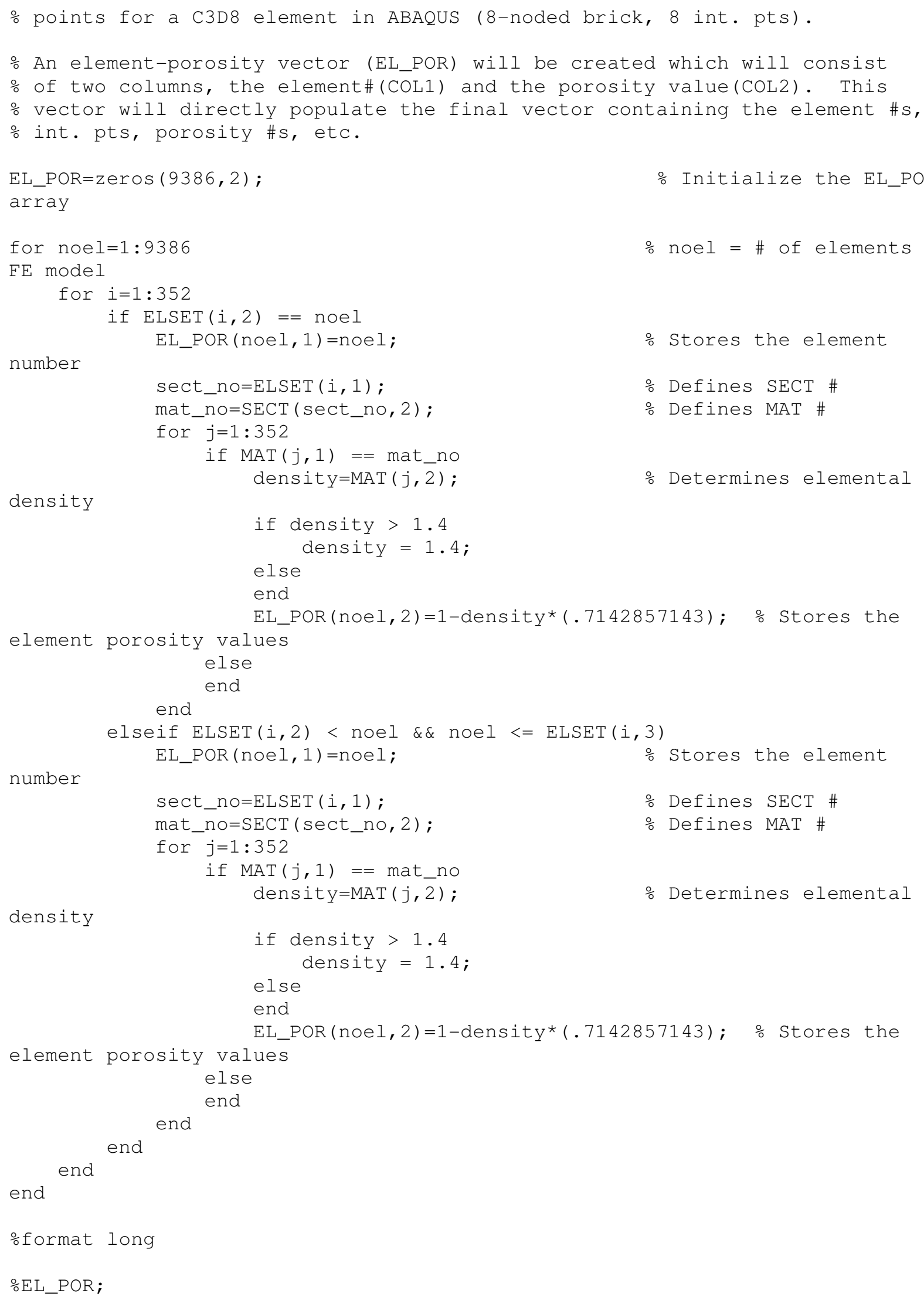


\% The final vector containg the elements, int. pts, and porosity values

\% will be stored as INT_POR. INT_POR will be a 75088x106 array with

\% element\#(COL1) and integration point\#(COL2). Colums 3 thru 106 correspond

\% to the dependent state variable in Dr. H's code. Below are listed the

\% definitions of the state variables along with their respective columns for

\% the final maxtix for a 4 step model (i.e. 4 loading cases in ABAQUS -

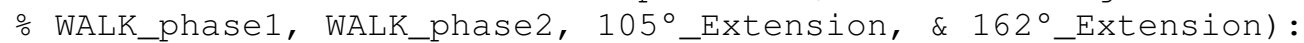

$\div \quad$ statev $(1)=$ porosity coL3

: statev (2) = strain (load case 1) COL4

\% statev(3) = strain (load case 2) COL5

: statev(4) = strain (load case 3) COL6

응 $\quad$ statev (5) = strain (load case 4) COL 7

: statev(6) = damage potential coL8

ㅇaㅇ $\quad$ statev (7) equilibrium damage COL9

o statev $(8)=$ \# of refilling BMUs COL10

\% statev $(9)=$ \# of resorbing BMUs COL11

\% statev $(10)=$ activation frequency COL12

응 statev(11) - statev(104) COL13 - CoL106

\% These colums correspond to the activation frequency history and

\% are directly related to the time for BMU resorption, reversal,

\% and refillig. For this particular model those times are 25, 5,

응 64 days respectively for a total of 94 days.

$\div$

Also, the 75088 is b/c its 8 int pts per each element so...

9386 elements $\times 8$ int pts $=75088$ lines of data.

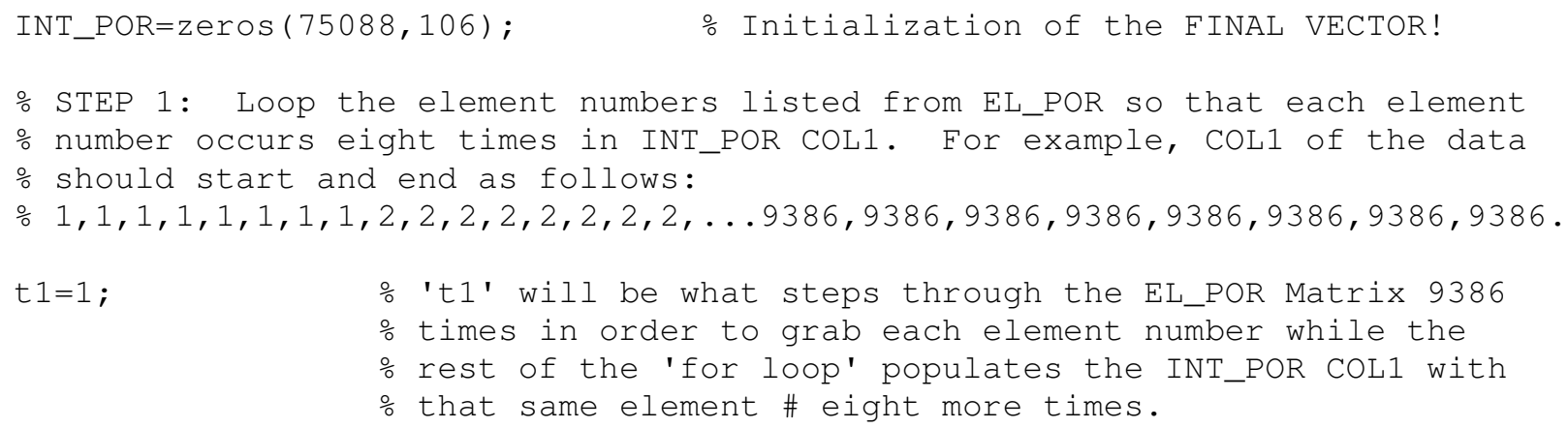

end

STEP 2: LOOP INT over COL2 of INT_POR array with the repeating 1-8 \#S

\% which represent the integration points per each element(COL1). For

\% example, COL2 should start and end like so:

응 $1,2,3,4,5,6,7,8,1,2,3, \ldots 4,5,6,7,8,1,2,3,4,5,6,7,8$.

INT=(1:8)'; $\quad$ 으 $\quad$ INT provides the Column Vector of \#s 1-8 which will

\% repeatedly be input into INT_POR COL2.

for el_int $=1: 8: 75088$

a $=$ el_int ; 


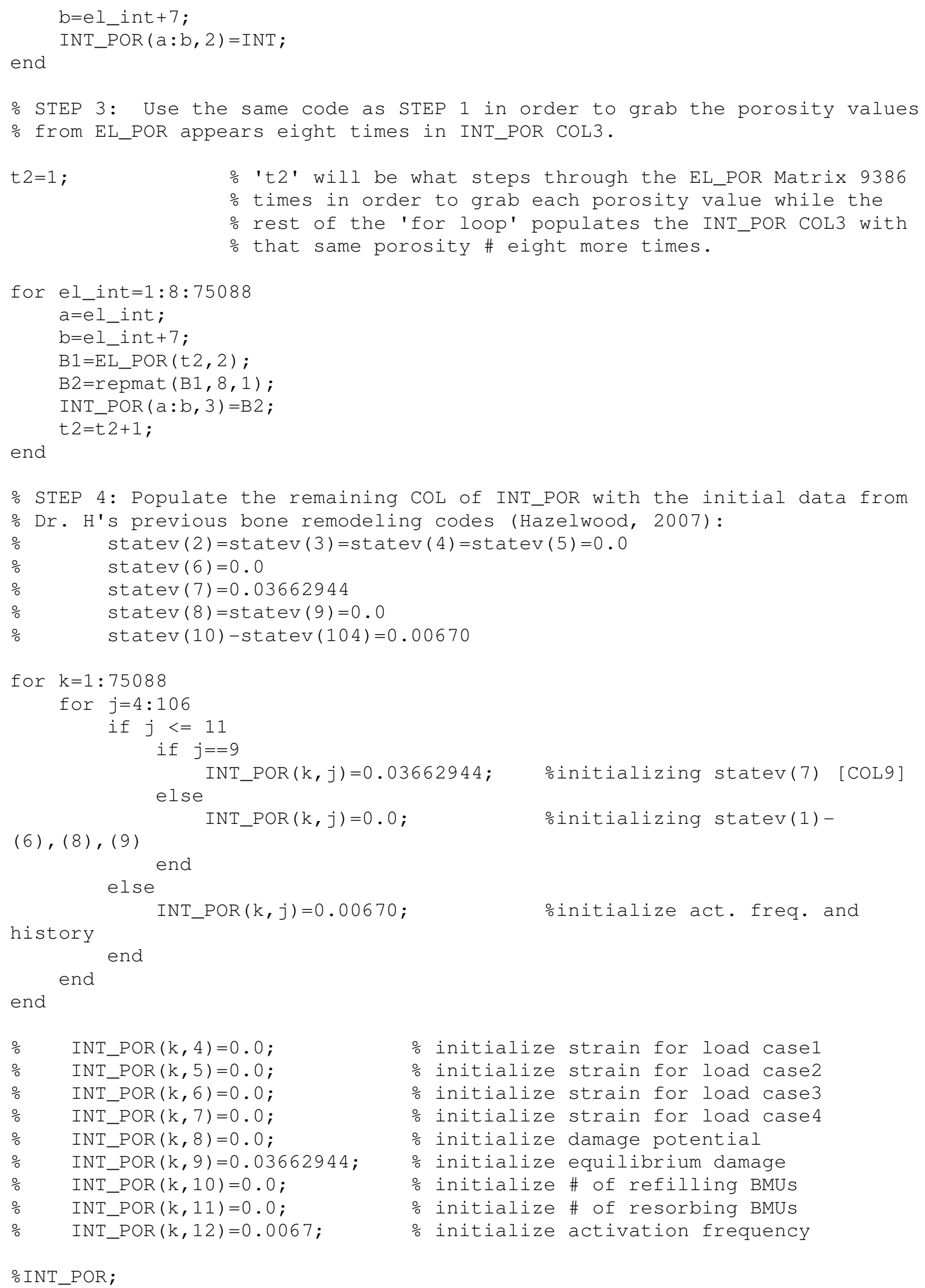




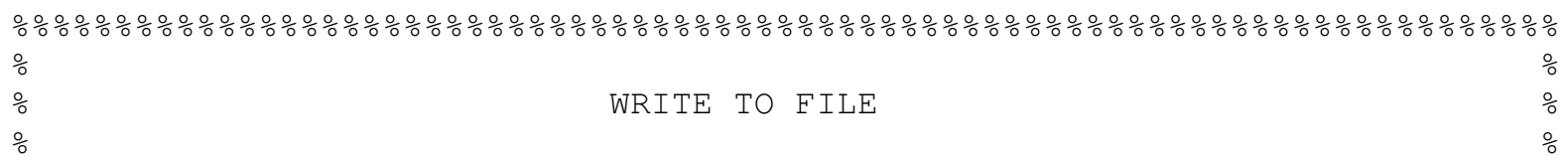

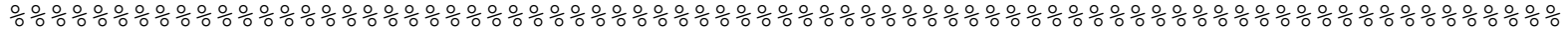

o The last thing to do is to write the INT_POR vector into a . txt file that \% will be able to be read directly by Dr. H's code. The last bit of code o will do precisely that.

INT_POR=INT_POR';

properly formatted

( $75088 \times 106$ array)

fid $=$ fopen('LegPreop3.txt', 'w');

\% fprintf formats the LegPreop.txt file, b/c there are 106 Columns

\% of data, there needs to be 106 formats specified in the statement \% below...

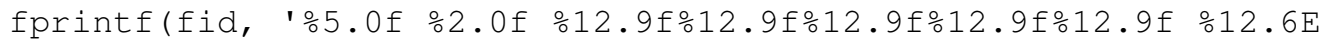

$\div 10.6 \mathrm{f} \div 10.6 \mathrm{f} \div 10.6 \mathrm{f}$

$\div 9.6 \mathrm{f} \div 9.6 \mathrm{f} \div 9.6 \mathrm{f} \div 9.6 \mathrm{f} \div 9.6 \mathrm{f} \div 9.6 \mathrm{f} \div 9.6 \mathrm{f} \div 9.6 \mathrm{f} \div 9.6 \mathrm{f} \div 9.6 \mathrm{f} \div 9.6 \mathrm{f} \div 9.6 \mathrm{f} \div 9.6 \mathrm{f} \div 9.6 \mathrm{f} \div 9.6 \mathrm{f} \div 9$ $.6 \mathrm{f} \div 9.6 \mathrm{f} \div 9.6 \mathrm{f} \div 9.6 \mathrm{f} \div 9.6 \mathrm{f} \div 9.6 \mathrm{f} \div 9.6 \mathrm{f} \div 9.6 \mathrm{f} \div 9.6 \mathrm{f} \div 9.6 \mathrm{f} \div 9.6 \mathrm{f} \div 9.6 \mathrm{f} \div 9.6 \mathrm{f} \div 9.6 \mathrm{f} \div 9.6 \mathrm{f} \div 9.6$ $\mathrm{f} \div 9.6 \mathrm{f} \div 9.6 \mathrm{f} \div 9.6 \mathrm{f} \div 9.6 \mathrm{f} \div 9.6 \mathrm{f} \div 9.6 \mathrm{f} \div 9.6 \mathrm{f} \div 9.6 \mathrm{f} \div 9.6 \mathrm{f} \div 9.6 \mathrm{f} \div 9.6 \mathrm{f} \div 9.6 \mathrm{f} \div 9.6 \mathrm{f} \div 9.6 \mathrm{f} \div 9.6 \mathrm{f} \%$ $9.6 \mathrm{f} \div 9.6 \mathrm{f} \div 9.6 \mathrm{f} \div 9.6 \mathrm{f} \div 9.6 \mathrm{f} \div 9.6 \mathrm{f} \div 9.6 \mathrm{f} \div 9.6 \mathrm{f} \div 9.6 \mathrm{f} \div 9.6 \mathrm{f} \div 9.6 \mathrm{f} \div 9.6 \mathrm{f} \div 9.6 \mathrm{f} \div 9.6 \mathrm{f} \div 9.6 \mathrm{f} \div 9$. $6 \mathrm{f} \div 9.6 \mathrm{f} \div 9.6 \mathrm{f} \div 9.6 \mathrm{f} \div 9.6 \mathrm{f} \div 9.6 \mathrm{f} \div 9.6 \mathrm{f} \div 9.6 \mathrm{f} \div 9.6 \mathrm{f} \% 9.6 \mathrm{f} \div 9.6 \mathrm{f} \div 9.6 \mathrm{f} \div 9.6 \mathrm{f} \div 9.6 \mathrm{f} \div 9.6 \mathrm{f} \div 9.6 \mathrm{f}$ $\div 9.6 \mathrm{f} \div 9.6 \mathrm{f} \div 9.6 \mathrm{f} \div 9.6 \mathrm{f} \div 9.6 \mathrm{f} \div 9.6 \mathrm{f} \div 9.6 \mathrm{f} \div 9.6 \mathrm{f} \div 9.6 \mathrm{f} \div 9.6 \mathrm{f} \div 9.6 \mathrm{f} \div 9.6 \mathrm{f} \div 9.6 \mathrm{f} \div 9.6 \mathrm{f} \div 9.6 \mathrm{f} \div 9$ $.6 \mathrm{f} \div 9.6 \mathrm{f} \div 9.6 \mathrm{f} \backslash \mathrm{n}^{\prime}$, INT_POR) ;

\%fprintf(fid, ' $\% 5.0 \mathrm{f} \div 2.0 \mathrm{f} \% 12.9 \mathrm{f} \% 12.9 \mathrm{f} \% 12.9 \mathrm{f} \% 12.9 \mathrm{f} \% 12.9 \mathrm{f} \% 12.9 \mathrm{f}$

$\circ 10.6 \mathrm{f} \div 10.6 \mathrm{f} \div 10.6 \mathrm{f}$

$\div 9.6 \mathrm{f} \div 9.6 \mathrm{f} \div 9.6 \mathrm{f} \div 9.6 \mathrm{f} \div 9.6 \mathrm{f} \div 9.6 \mathrm{f} \div 9.6 \mathrm{f} \div 9.6 \mathrm{f} \div 9.6 \mathrm{f} \div 9.6 \mathrm{f} \div 9.6 \mathrm{f} \div 9.6 \mathrm{f} \div 9.6 \mathrm{f} \div 9.6 \mathrm{f} \div 9.6 \mathrm{f} \div 9$ $.6 \mathrm{f} \div 9.6 \mathrm{f} \div 9.6 \mathrm{f} \div 9.6 \mathrm{f} \div 9.6 \mathrm{f} \div 9.6 \mathrm{f} \div 9.6 \mathrm{f} \div 9.6 \mathrm{f} \div 9.6 \mathrm{f} \div 9.6 \mathrm{f} \div 9.6 \mathrm{f} \div 9.6 \mathrm{f} \div 9.6 \mathrm{f} \div 9.6 \mathrm{f} \div 9.6 \mathrm{f} \div 9.6$ $\mathrm{f} \div 9.6 \mathrm{f} \div 9.6 \mathrm{f} \div 9.6 \mathrm{f} \div 9.6 \mathrm{f} \div 9.6 \mathrm{f} \div 9.6 \mathrm{f} \div 9.6 \mathrm{f} \div 9.6 \mathrm{f} \div 9.6 \mathrm{f} \div 9.6 \mathrm{f} \div 9.6 \mathrm{f} \div 9.6 \mathrm{f} \div 9.6 \mathrm{f} \div 9.6 \mathrm{f} \div 9.6 \mathrm{f} \%$ $9.6 \mathrm{f} \div 9.6 \mathrm{f} \div 9.6 \mathrm{f} \div 9.6 \mathrm{f} \div 9.6 \mathrm{f} \div 9.6 \mathrm{f} \div 9.6 \mathrm{f} \div 9.6 \mathrm{f} \div 9.6 \mathrm{f} \div 9.6 \mathrm{f} \div 9.6 \mathrm{f} \div 9.6 \mathrm{f} \div 9.6 \mathrm{f} \div 9.6 \mathrm{f} \div 9.6 \mathrm{f} \div 9$. $6 \mathrm{f} \div 9.6 \mathrm{f} \div 9.6 \mathrm{f} \div 9.6 \mathrm{f} \div 9.6 \mathrm{f} \div 9.6 \mathrm{f} \div 9.6 \mathrm{f} \div 9.6 \mathrm{f} \div 9.6 \mathrm{f} \div 9.6 \mathrm{f} \div 9.6 \mathrm{f} \div 9.6 \mathrm{f} \div 9.6 \mathrm{f} \div 9.6 \mathrm{f} \div 9.6 \mathrm{f} \div 9.6 \mathrm{f}$ $\div 9.6 \mathrm{f} \div 9.6 \mathrm{f} \div 9.6 \mathrm{f} \div 9.6 \mathrm{f} \div 9.6 \mathrm{f} \div 9.6 \mathrm{f} \div 9.6 \mathrm{f} \div 9.6 \mathrm{f} \div 9.6 \mathrm{f} \div 9.6 \mathrm{f} \div 9.6 \mathrm{f} \div 9.6 \mathrm{f} \div 9.6 \mathrm{f} \div 9.6 \mathrm{f} \div 9.6 \mathrm{f} \div 9$ $.6 \mathrm{f} \div 9.6 \mathrm{f} \div 9.6 \mathrm{f} \backslash \mathrm{n}^{\prime}$, INT_POR) ;

fclose (fid);

otype LegExt_IntPor.txt $\quad$ 'type' outputs the .txt data in the MATLAB command window

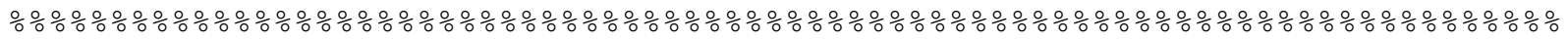
CODE SUCCESSFULLY RUN ON 5-9-2010 AND OUTPUT ALL 106 COLUMNS WHICH WERE \% THEN VERIFIED AGAINST THE ORIGINAL ABAQUS . INP FILE.

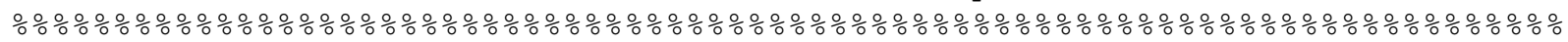




\section{APPENDIX C}

\section{ABAQUS INPUT FILE FOR THE MUSCLE FORCES OF THE FEMUR}




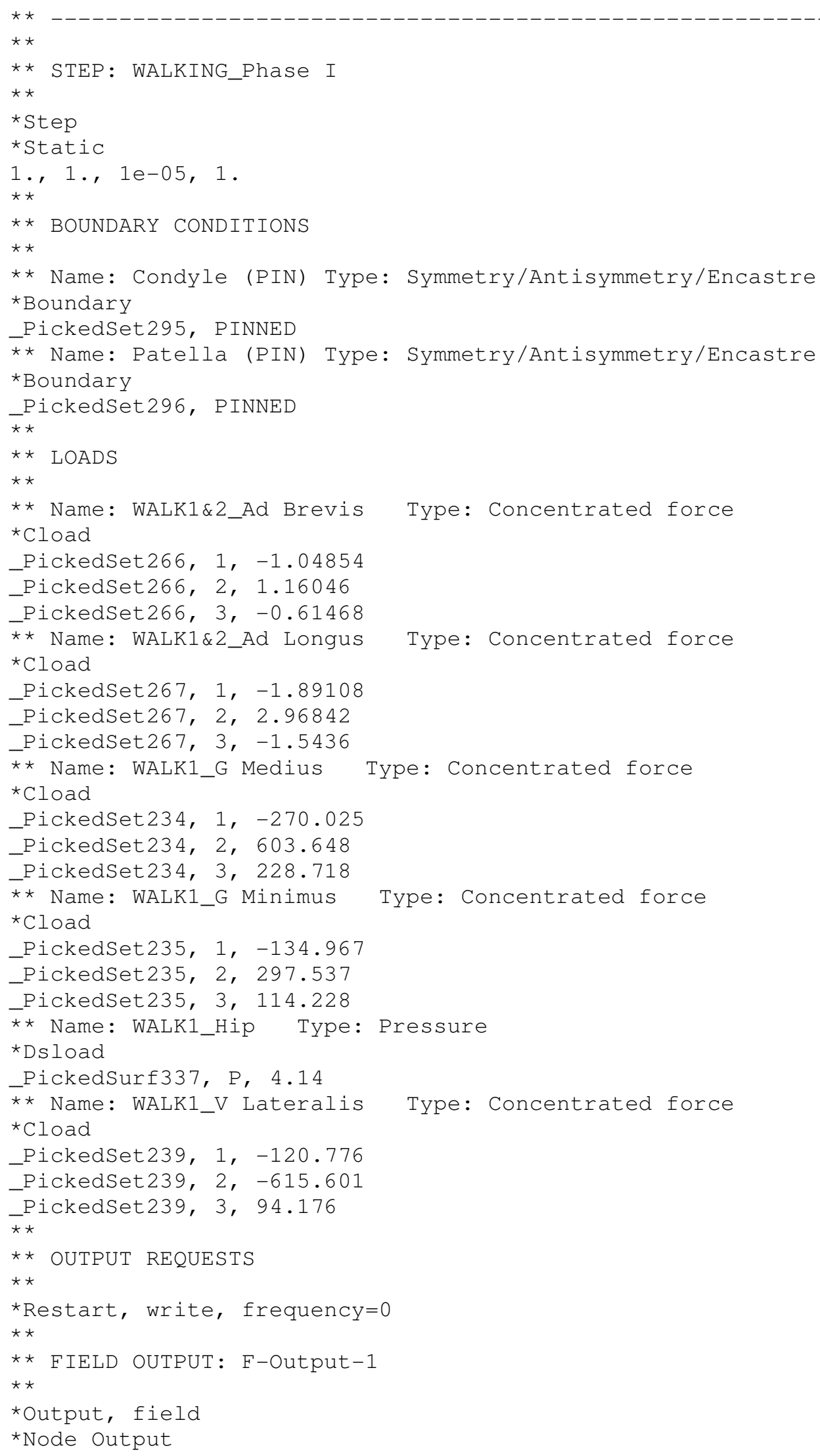




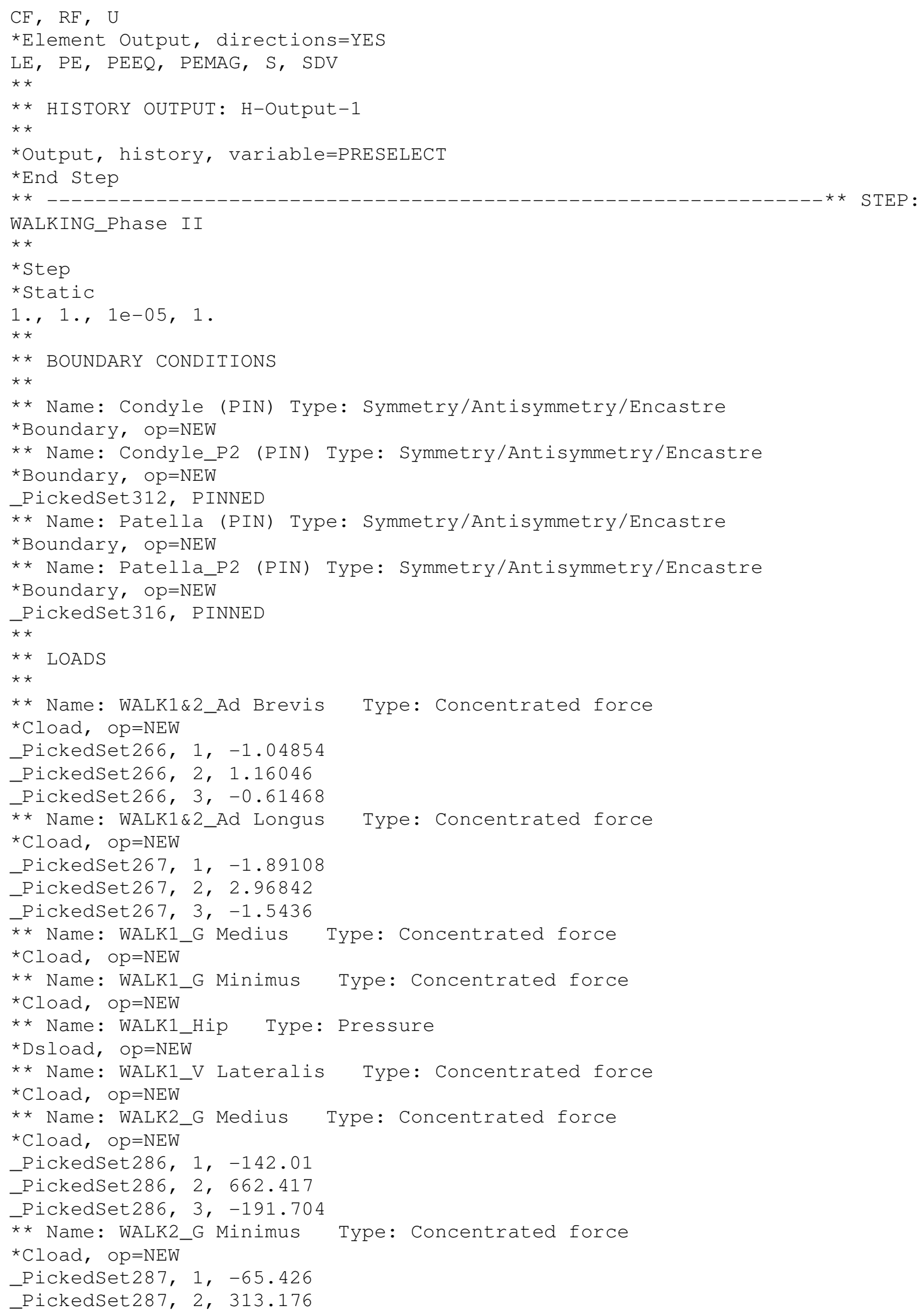




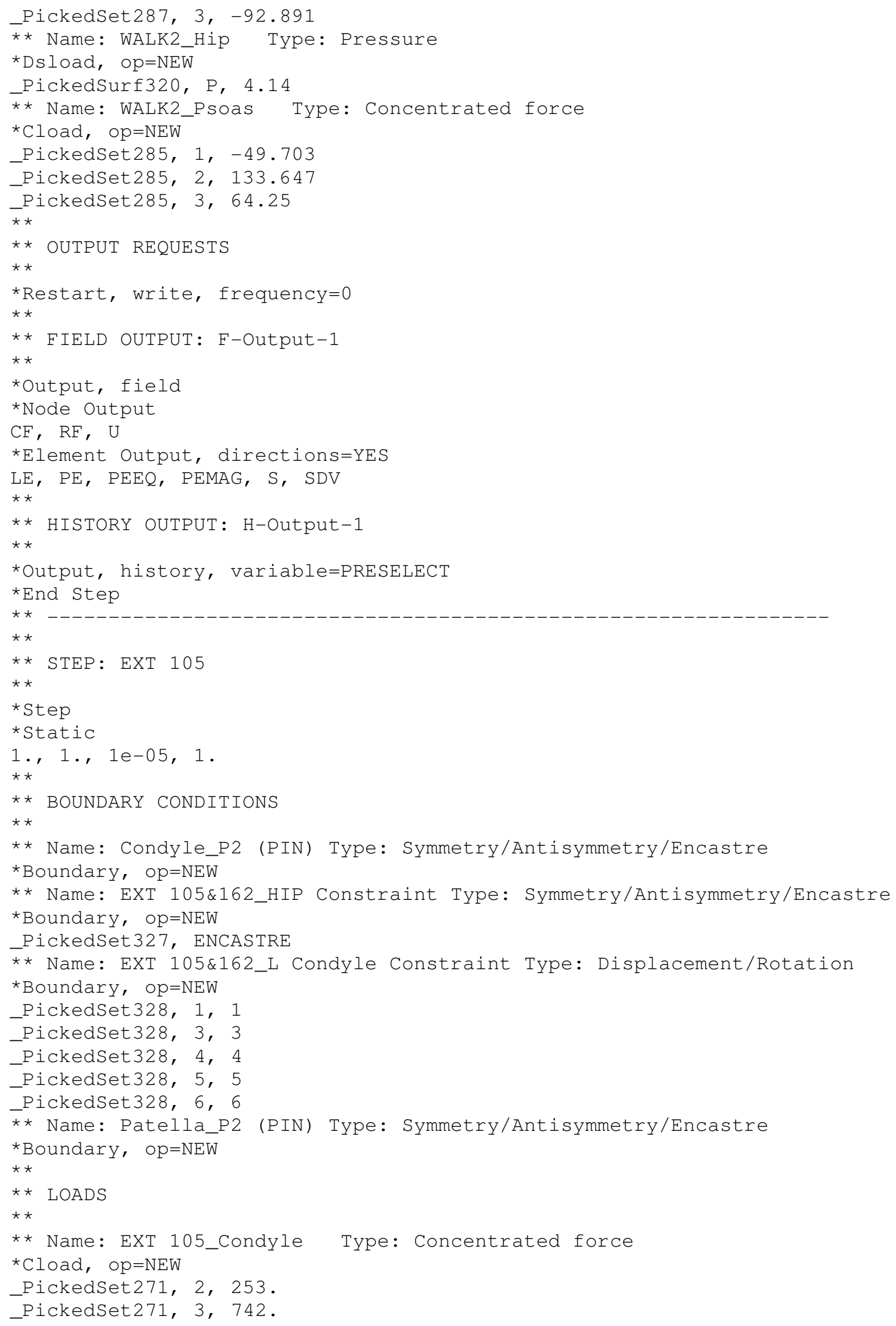




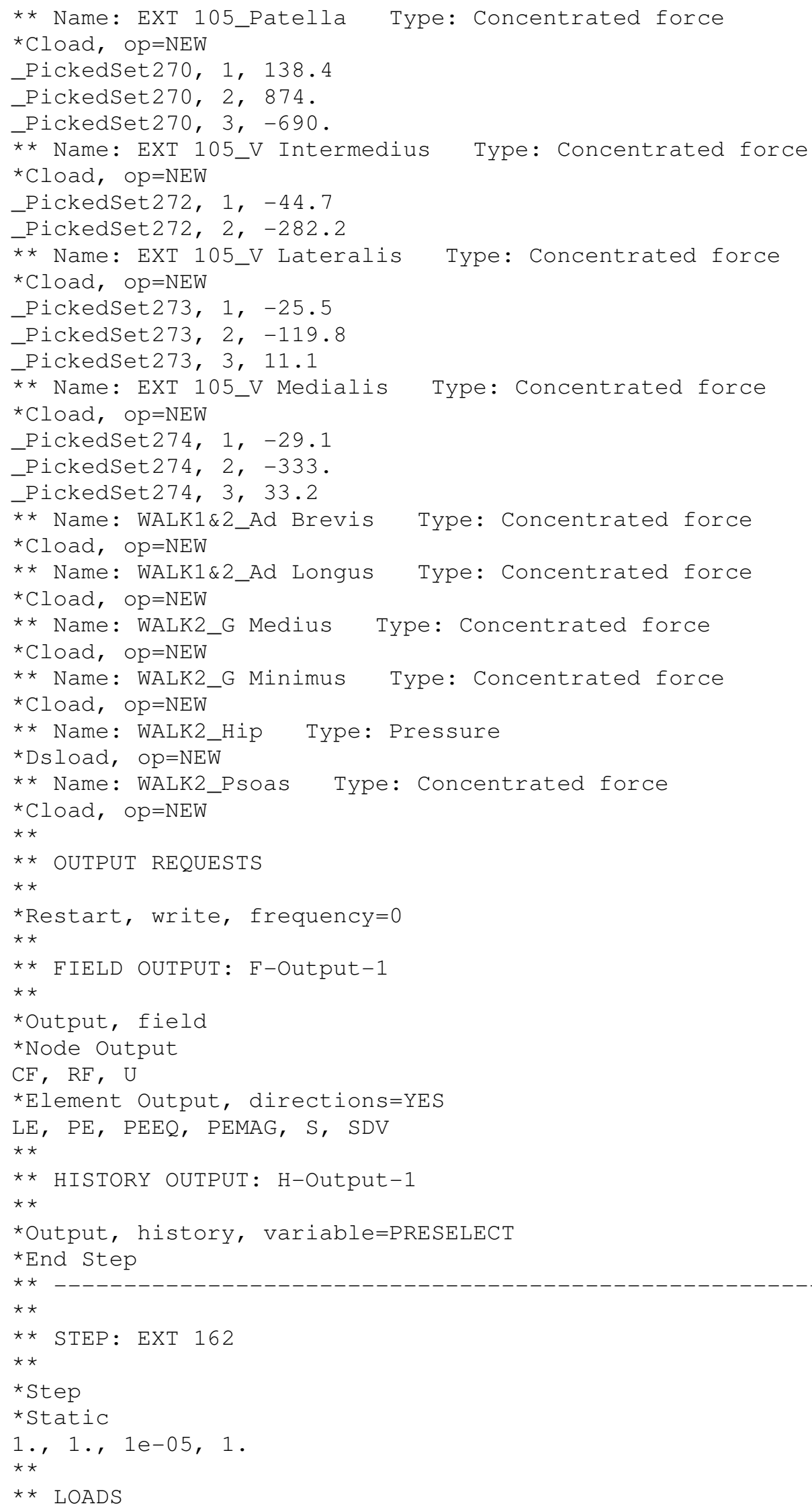




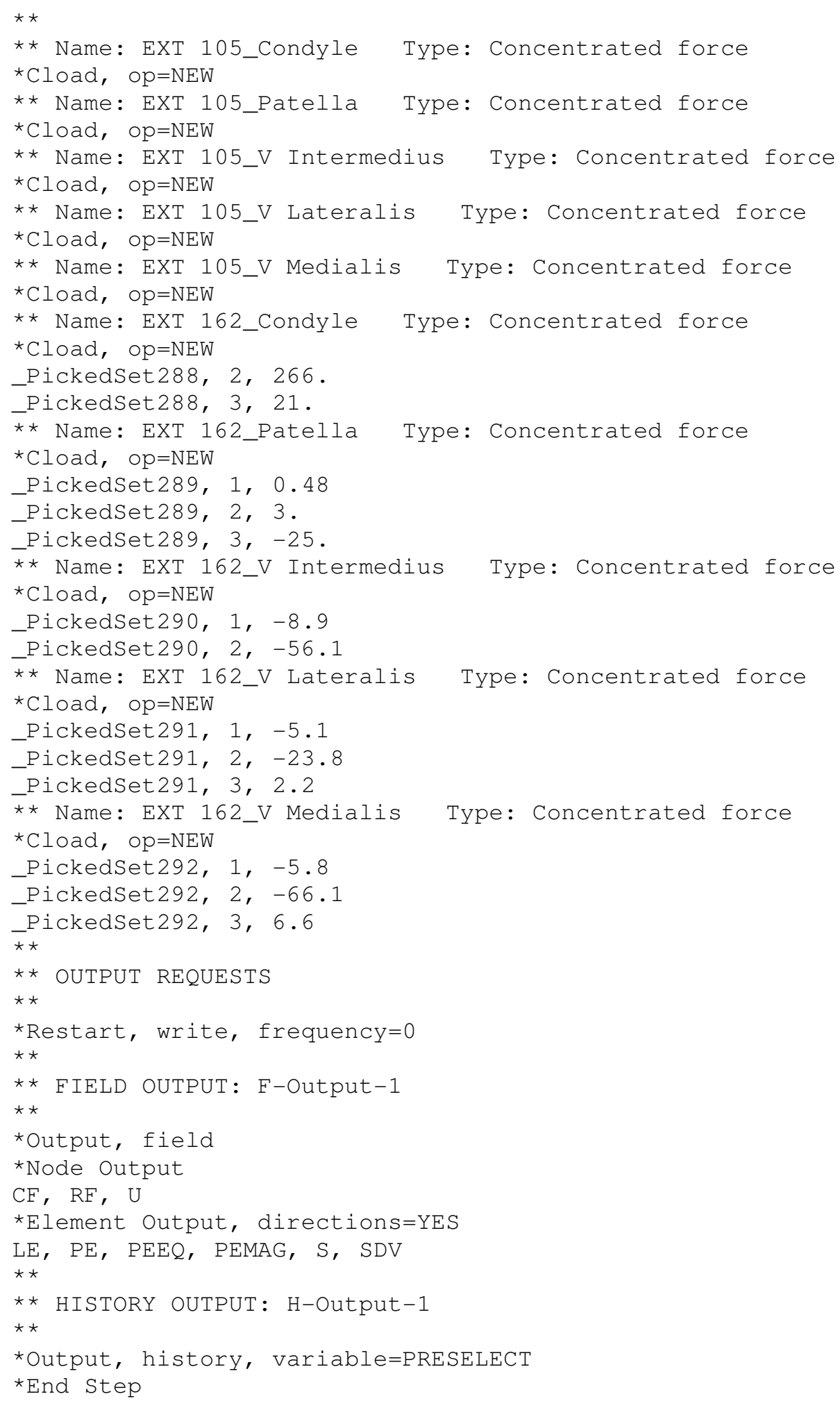




\section{APPENDIX D}

\section{HOW TO CREATE A UMAT IN ABAQUS}




\section{CREATING A WORKING UMAT INPUT FILE IN ABAQUS}

(SPECIFICALLY FOR DR. HAZELWOODS BONE REMODELING UMAT)

1 OPEN ABAQUS CAE: Open the Graphical User Interface (GUI) for ABAQUS.

2 PART MODULE (ABAQUS): Create/Import part (this example used Orphan Mesh of a femur.)

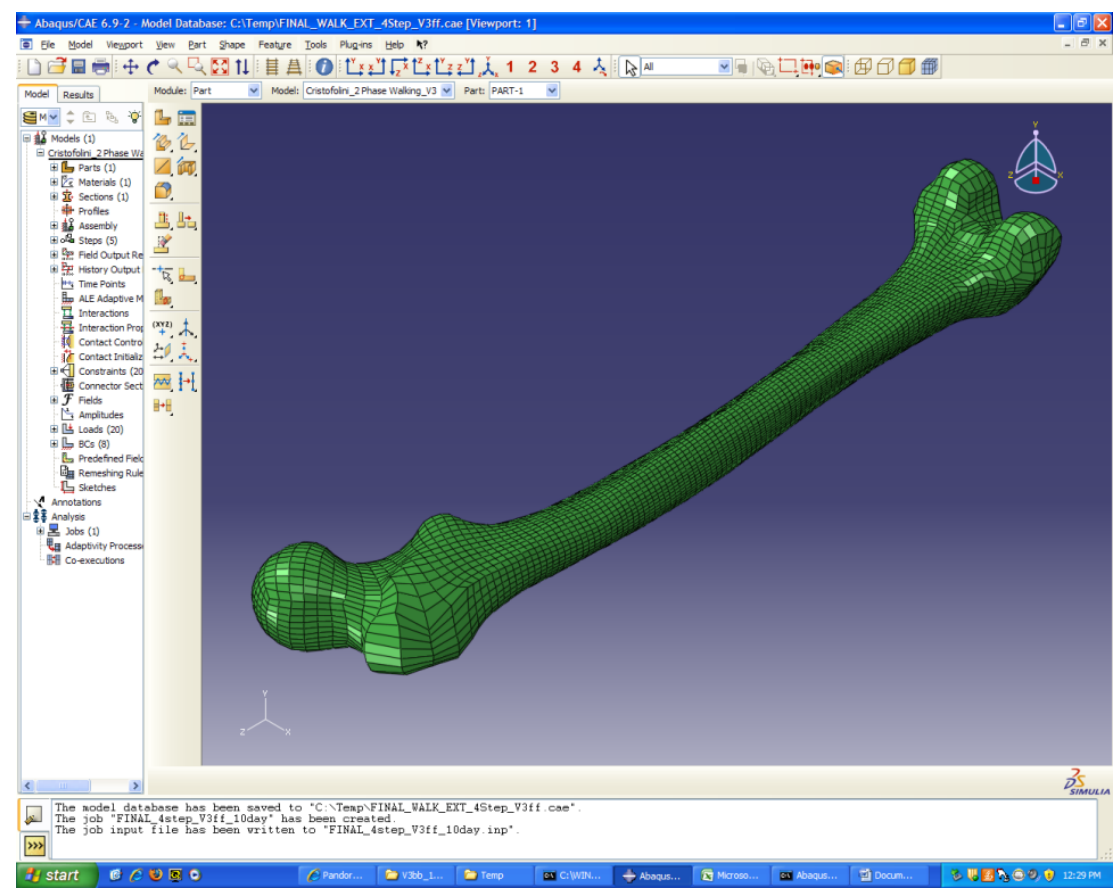

3 PROPERTY MODULE (ABAQUS): Create and assign the User Material (UMAT)

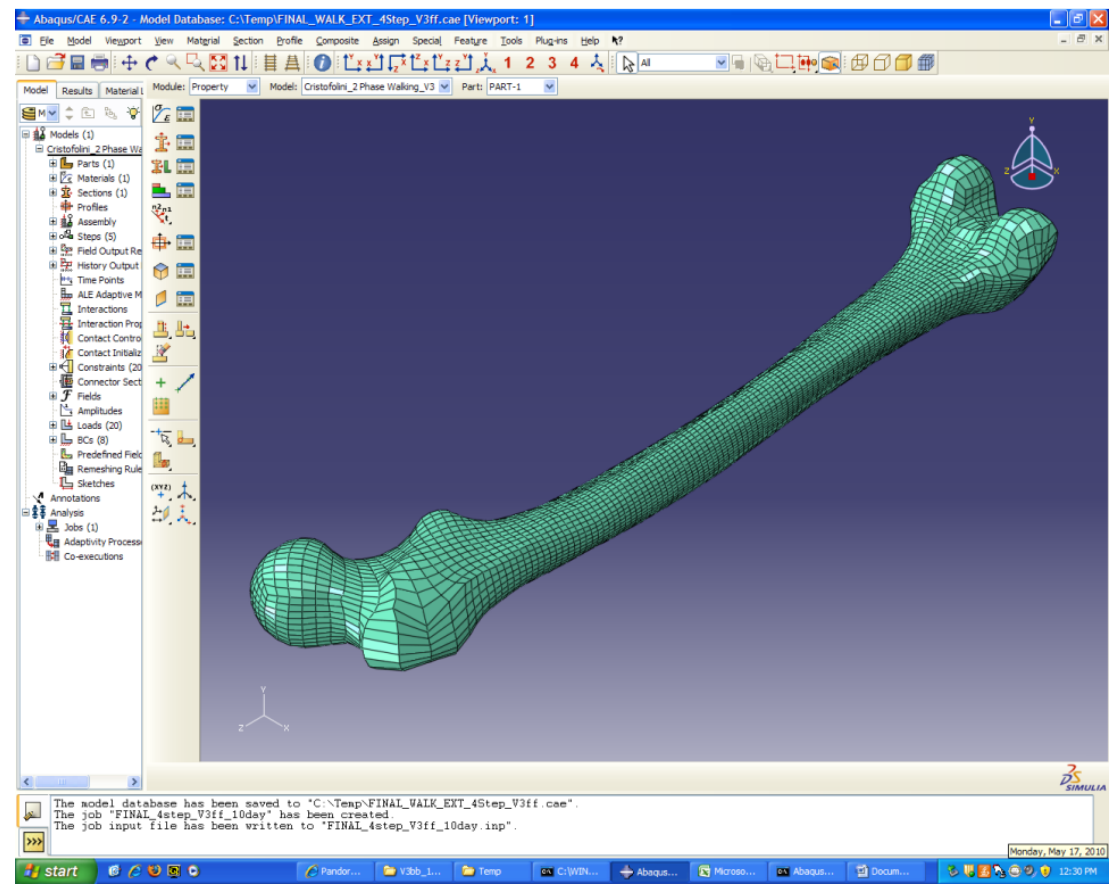


a. Property Manager: Create a new Material.

i. Open the Property Manager and Click on the Create button. Name the material (BONE in this example).

ii. Next, Click on the General tab and select the Depvar option.

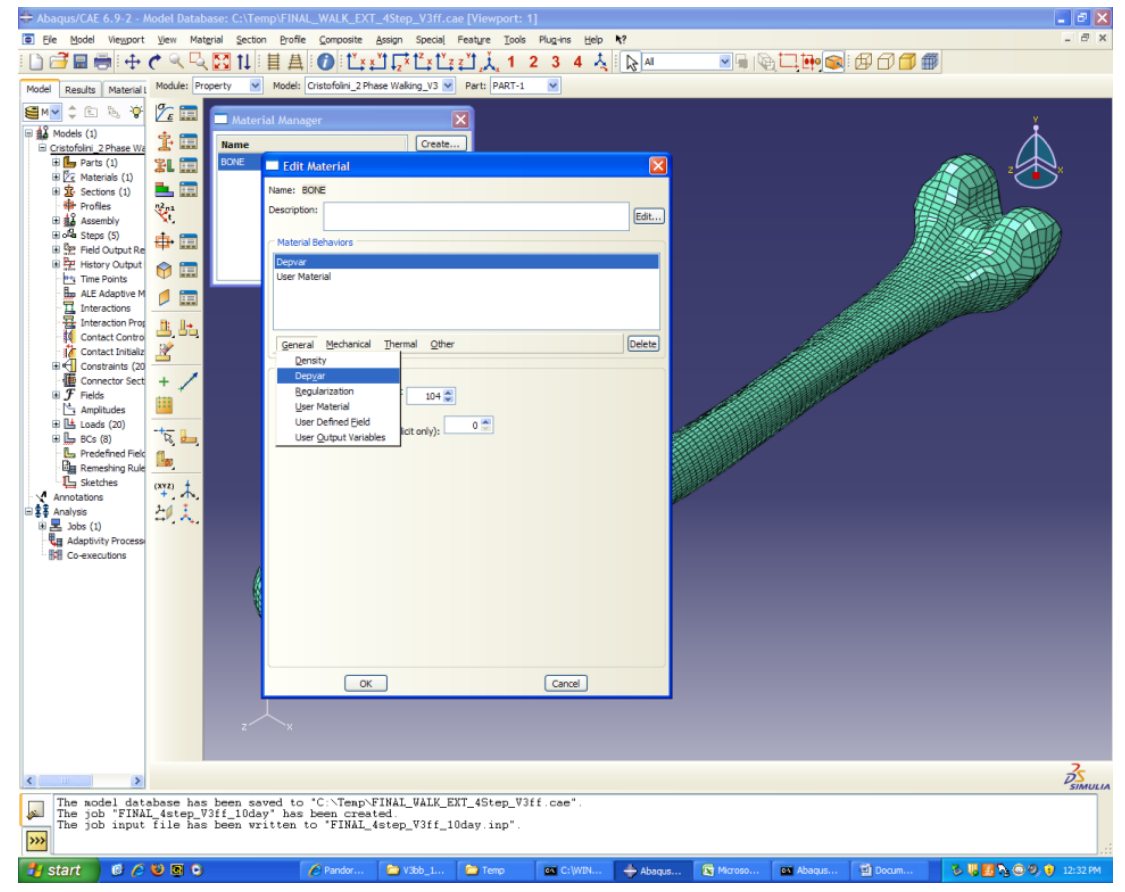

iii. For the Number of solution dependent state variables: field input 104 (this value can vary depending on the type of simulation (i.e. 3 step or 4 step), therefore check the particular bone UMAT file and check the \# of variables that the code calls for)

iv. Leave the other field blank (only applies to ABAQUS/Explicit) and Click OK. (see below for example of a proper Depvar material GUI)

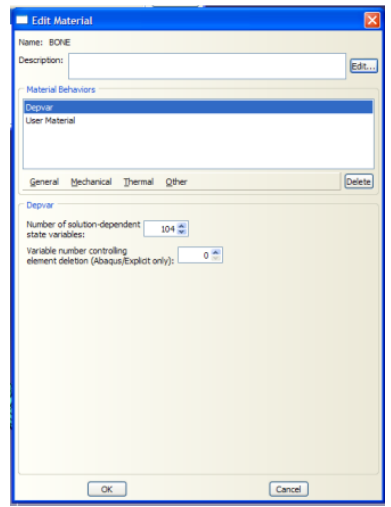

v. Finally, Click on the General tab and select the User Material option. 


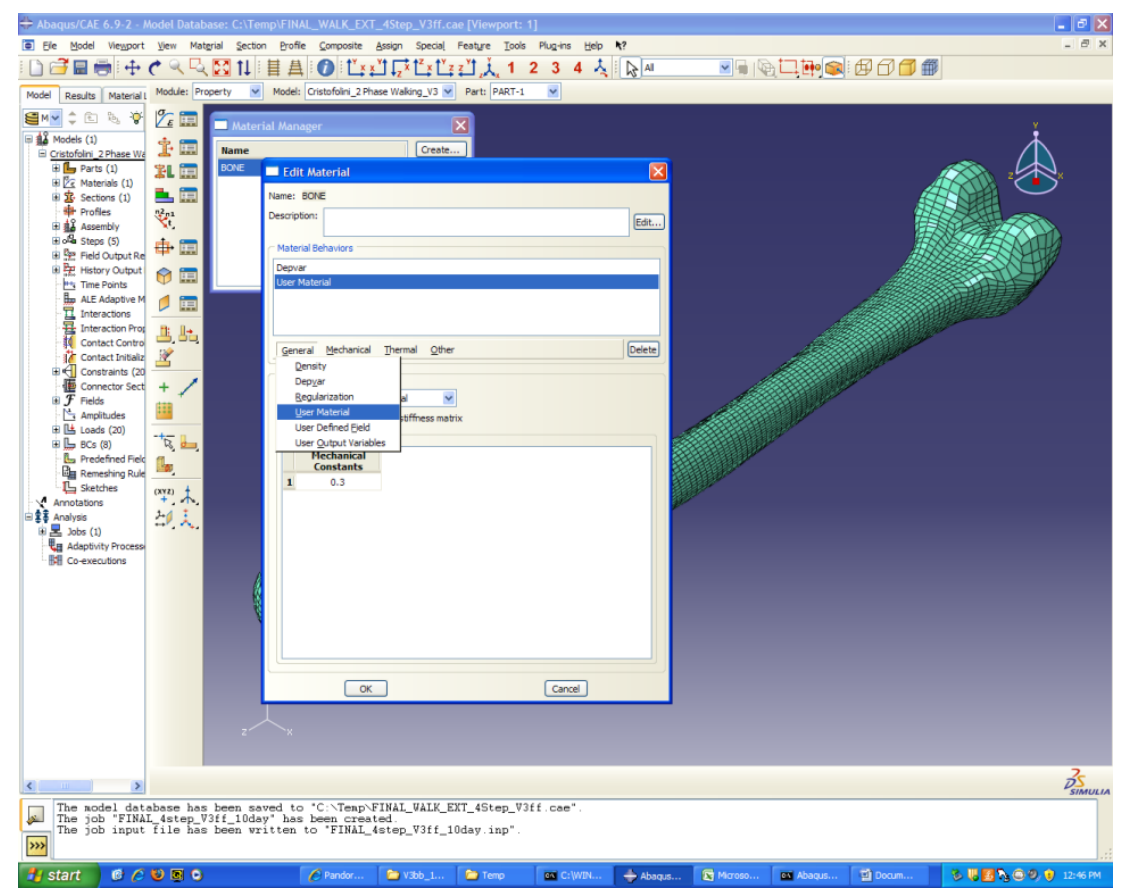

vi. In the User material type: field, make sure that Mechanical is selected (also, leave Use unsymmetric material stiffness matrix box blank.)

vii. In the Mechanical Constants field, make sure only one data cell is selected and input a value of $\mathbf{0 . 3}$. (This value represents the Poisson's Ratio for the bone material.)

viii. Click OK. (see below for example of a proper User Material material GUI)

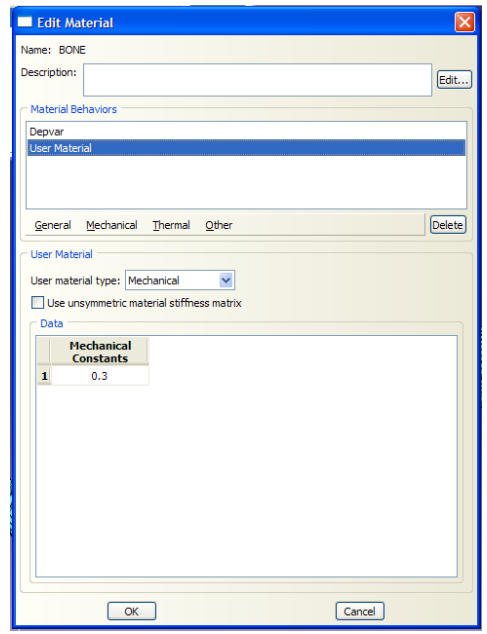

b. Section Manager: Create a Section for the new material.

i. Open the Section Manager and Click the Create button.

ii. Name the section (BONE in this example) and select the Solid and

Homogeneous options and then Click Continue... . (see below) 


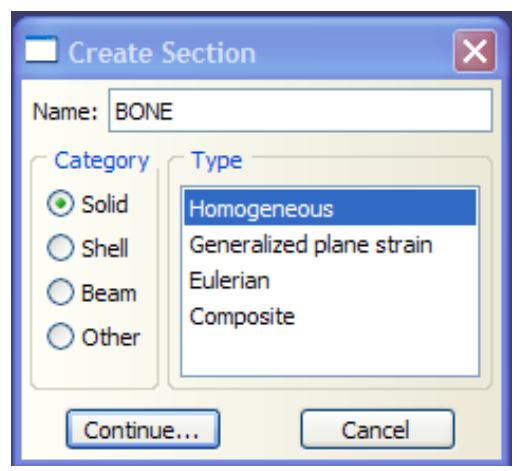

iii. Use the pull down menu to select the BONE material created previously (part a) and Click $O K$ to finish. (see below)

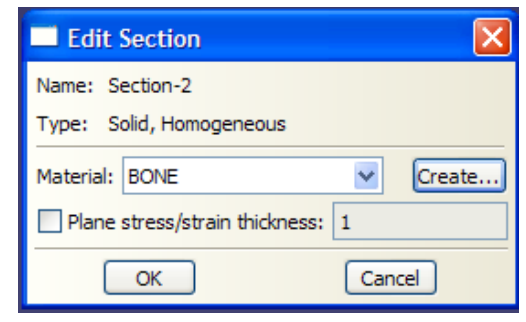

c. Section Assignment Manager: Assign the Section to the Part.

i. Open the Section Assignment Manager and Click the Create button.

ii. Manually select the entire Part and Click the Done button.

iii. Use the pull down menu to select the BONE Section created previously (part b) and Click OK to finish. (see below)

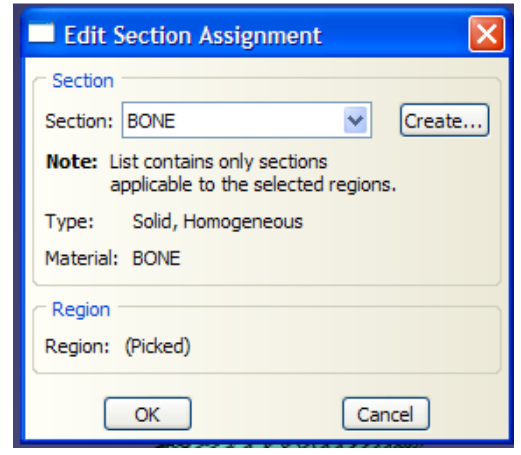

4 ASSEMBLY MODULE (ABAQUS): Instance the Part. (Same as in normal ABAQUS FE models) 5 STEP MODULE (ABAQUS): Create the loading conditions (this example model had 4 Steps) 


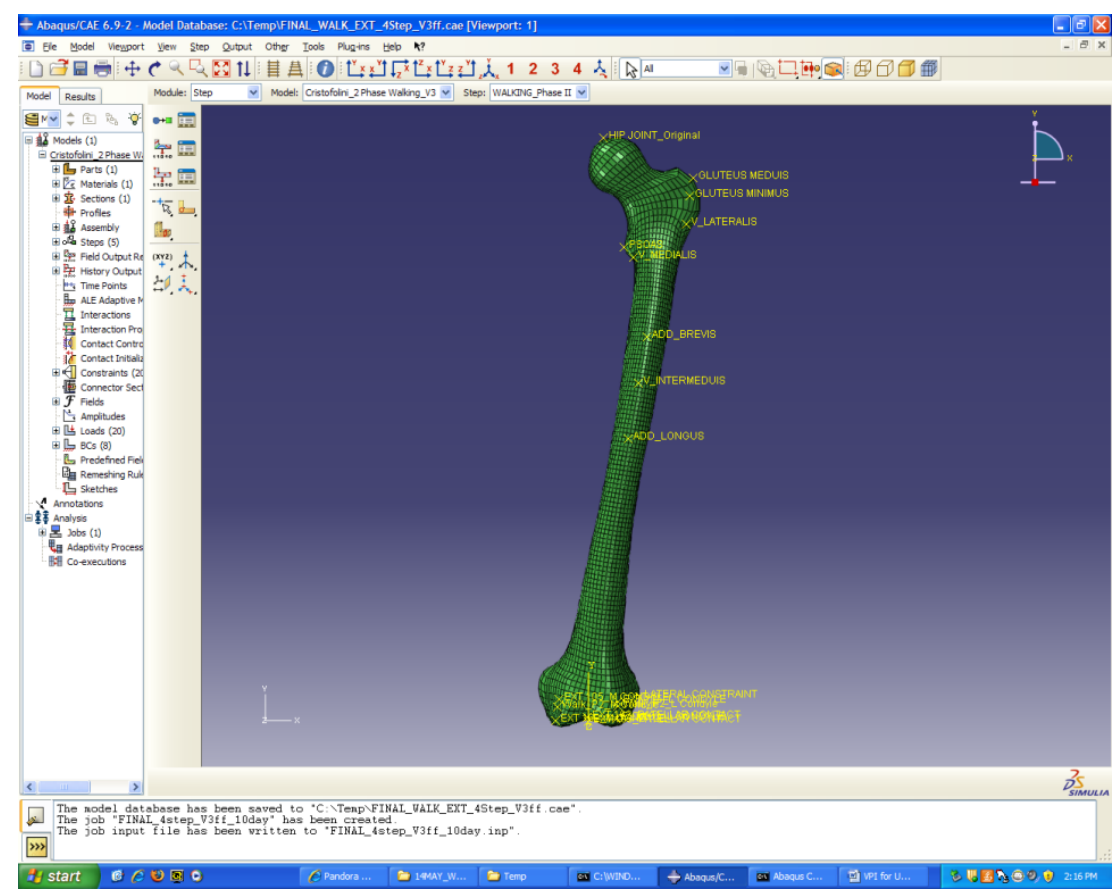

a. Field Output Request Manager: Edit the Field Output to include the UMAT dependent state variables.

i. Open the Field Output Request Manager and Click the Edit button (a default field should already exist called F-Output-1, if it does not Click the Create button and follow the steps below).

ii. From the Output Variables List, scroll down and select the State/Field/User/Time Option and then make sure the SDV box is checked. (see below) 


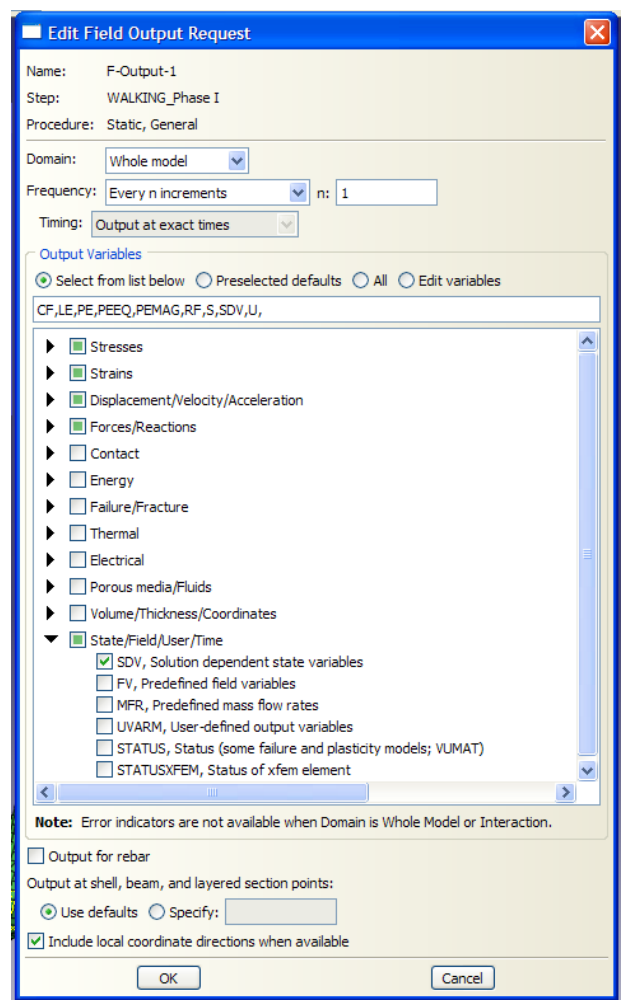

6 INTERACTION MODULE (ABAQUS): Create the required interactions (if any) required for the model.

7 LOAD MODULE (ABAQUS): Apply the required loads for the model.

8 MESH MODULE (ABAQUS): Mesh the model (not requ'd if an Orphan Meshed Part is used).

9 JOB MODULE (ABAQUS): Create the job required for ABAQUS to perform the analysis.

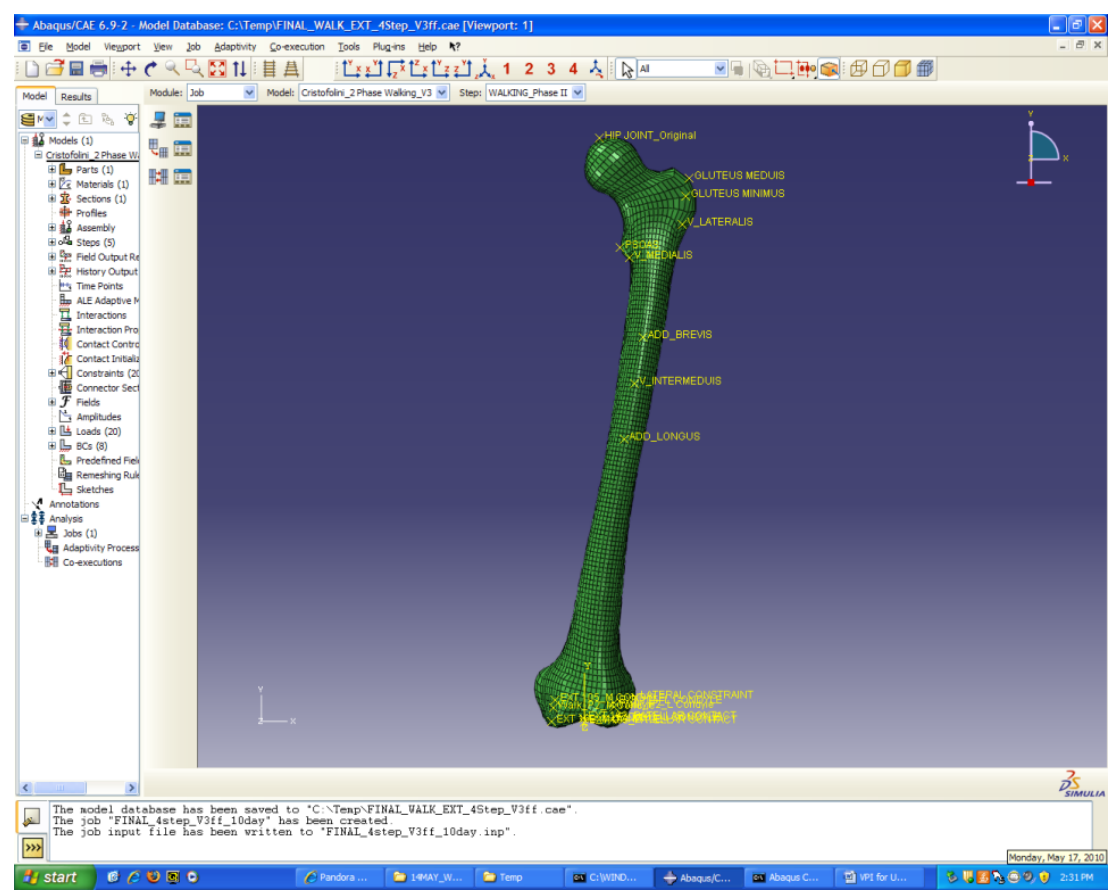


a. Job Manager: Create the Input File (.inp file) needed for running the analysis.

i. Open the Job Manager and Click the Create button.

ii. Name the job accordingly and Click the Continue... button.

iii. Select the General tab and under the User subroutine file: section Click the Select... button and use the browse feature to select the UMAT file (the file needs to be in the _.for format).

1. **IMPORTANT: The UMAT file needs to be placed into whatever directory the $A B A Q U S$ job is run (for current example, the UMAT file is located in the C: (Temp directory)**

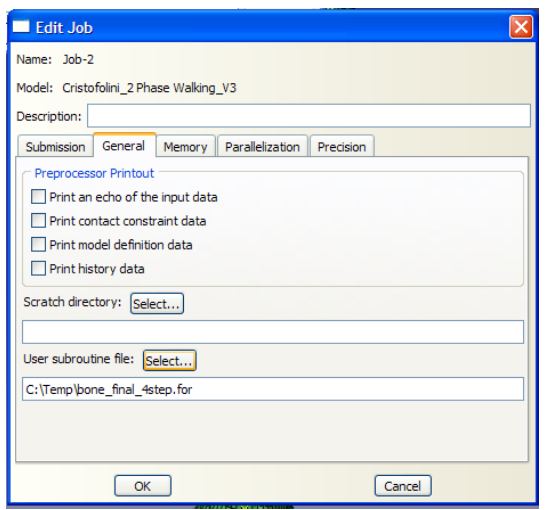

iv. Click the $\boldsymbol{O} \boldsymbol{K}$ button to complete the job.

v. At this point the job that was just created should be listed in the Job Manager. Highlight the job (single Click), and then Click on the Write Input button. This will create the Input file (_.inp) in the corresponding ABAQUS directory. (see below).

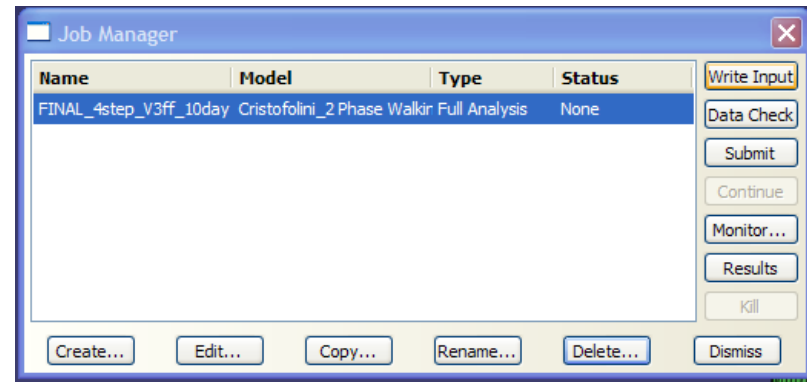

10 INPUT FILE MANIPULATION: Manually edit the Input File.

a. Open Input File:

i. Go to the directory which the Input file was created and Open the .inp file.

b. Material Initialization:

i. Scroll down until you find the ** Materials section of the input file.

ii. After the last bit of Material information the following line of code needs to be written into the file: * Initial Conditions, type=Solution, user. (see below for a before(Left) and after(Right) version of the .inp file) 

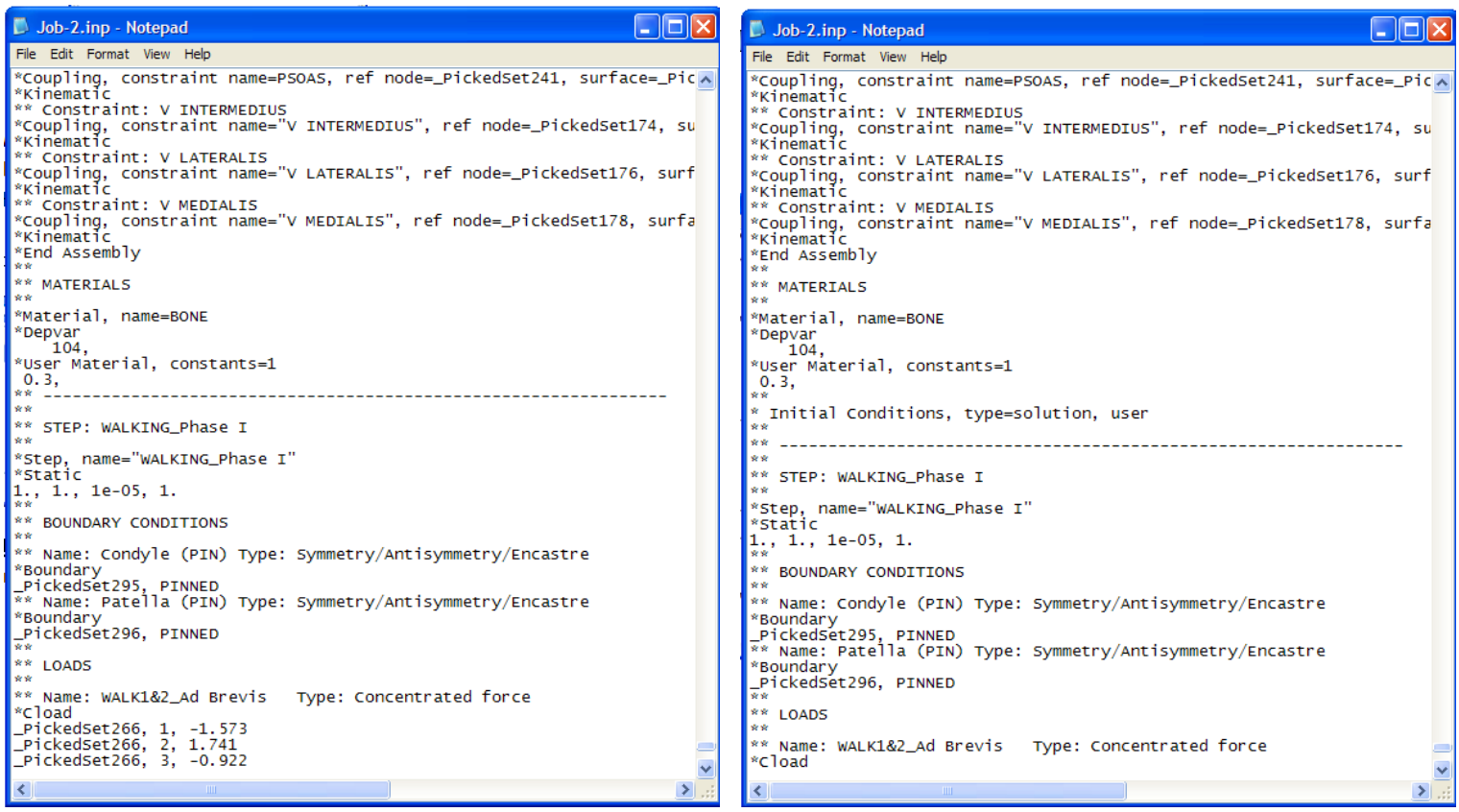

\section{c. Remove STEP Names:}

i. If the step names are not removed then the code will not run properly. (Note: any line of code beginning with ${ }^{* *}$ does not need to be modified as it will not be read by ABAQUS $b / c$ it denotes a comment). Therefore, only the lines of code with * need to be modified.

ii. Locate the STEP data, which is typically contained immediately after the Material data.

iii. Edit the line of code *Step, name="__ " to read *Step only. (see below for a before (Left) and after (Right) version of the inp file)

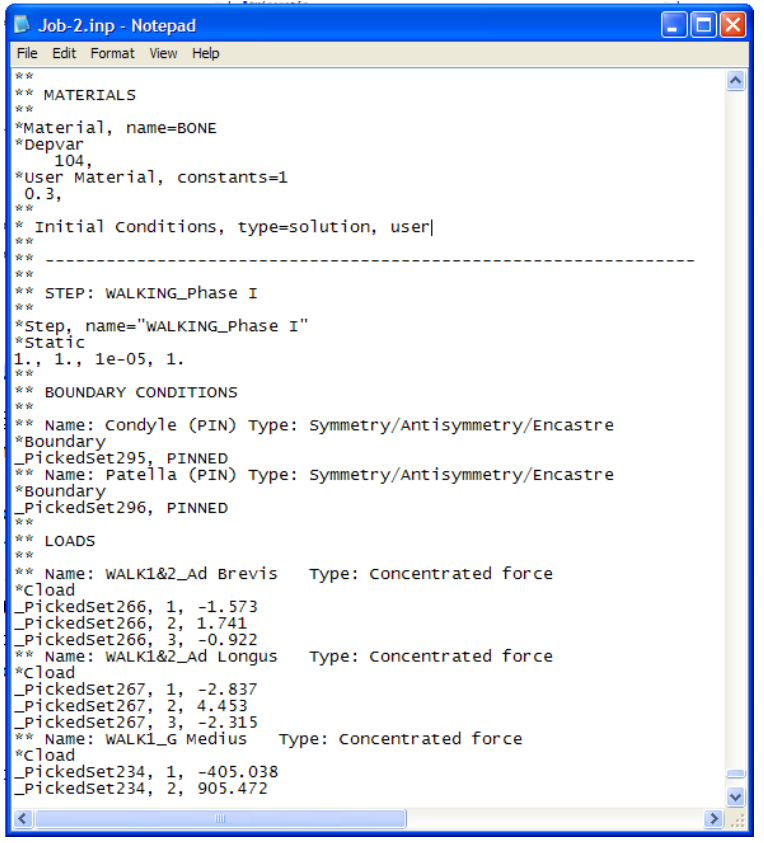

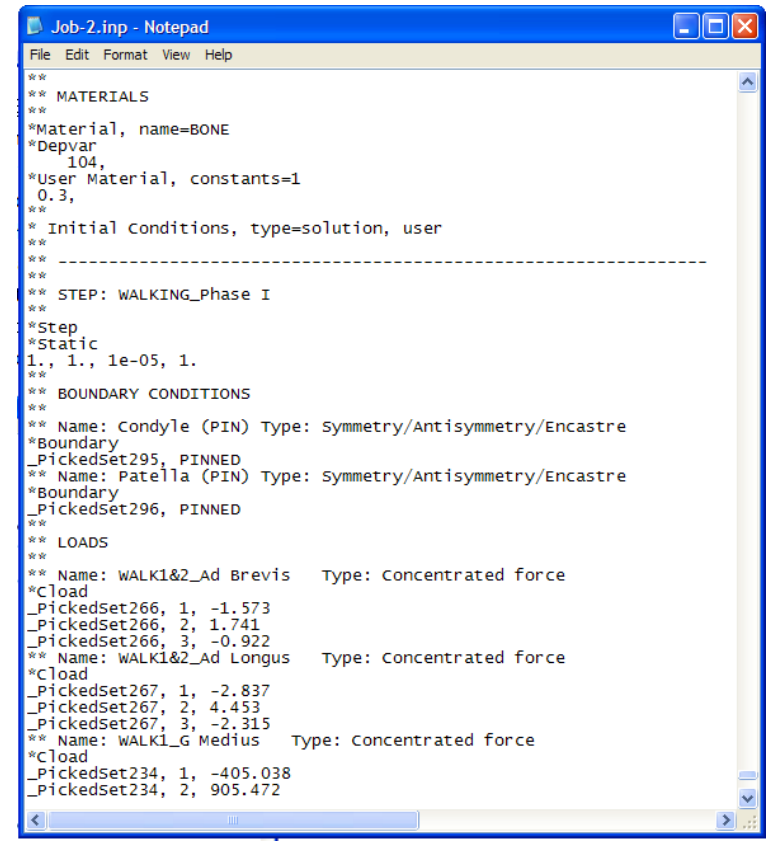


iv. Note: this must be done for every STEP. If there were three STEPS defined in the model then all three need to be changed as described above.

d. Manipulate length of Simulation:

i. The bone remodeling code by Dr. Hazelwood is set up to calculate and determine results every day. Each STEP is run consecutively with the last step triggering the remodeling analysis [i.e. every time unit (day) each STEP is run once]. Therefore, if you had a four STEP model and wanted to run a 10 day simulation you would need to duplicate the four STEPs in the inp file nine more times.

ii. This is as simple as cutting and pasting the group of four STEPS in the inp code.

iii. CAUTION: If you do not copy and paste the complete set of four STEPS then the model will not run properly.

11 JOB SUBMISSION: Submitting the job through the ABAQUS COMMAND window (in Windows)

a. Open the ABAQUS COMMAND window.

b. Set the working directory to where you want (in this example $\mathrm{C}: \backslash T e m p$ )

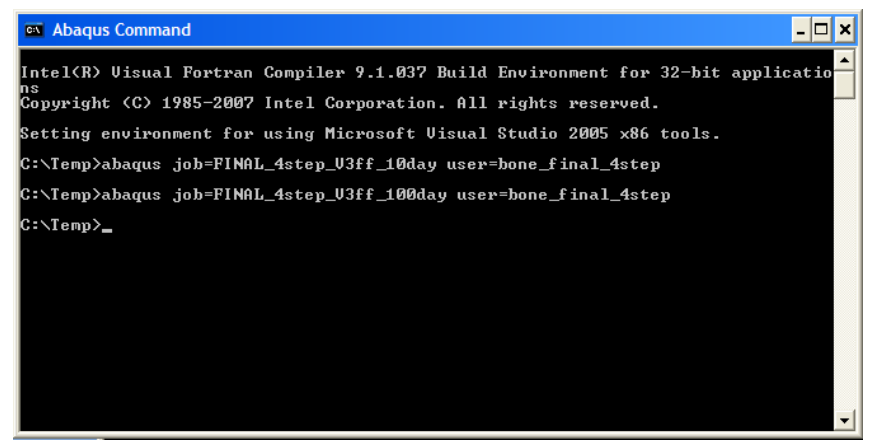

c. Type in the following command to run the analysis: abaqus job="job name" user="UMAT file name"

i. NOTE: the job name and UMAT file name are not in quotes and the spaces between the commands are necessary.

ii. EXAMPLE: abaqus job=FINAL_4step_V3 user=bone_final_4step

d. An output database file (.odb) of the ABAQUS analysis will be created in the directory chosen. This file can be opened via ABAQUS CAE in order to visually see the results from the analysis. 